\title{
Terrasses aux mosaïques de Choqek'iraw, Pérou. Description générale et premières interprétations
}

Patrice Lecoq

\section{(2) OpenEdition}

\section{Journals}

Édition électronique

URL : https://journals.openedition.org/jsa/11497

DOI : 10.4000/jsa. 11497

ISSN : $1957-7842$

Éditeur

Société des américanistes

\section{Édition imprimée}

Date de publication : 5 décembre 2010

Pagination : 7-73

ISSN : 0037-9174

\section{Référence électronique}

Patrice Lecoq, «Terrasses aux mosaïques de Choqek'iraw, Pérou. Description générale et premières interprétations », Journal de la Société des américanistes [En ligne], 96-2 | 2010, mis en ligne le 10 décembre 2014, consulté le 04 septembre 2022. URL : http://journals.openedition.org/jsa/11497 ; DOI : https://doi.org/10.4000/jsa. 11497 


\title{
TERRASSES AUX MOSAÏQUES DE CHOQEK'IRAW, PÉROU. DESCRIPTION GÉNÉRALE ET PREMIÈRES INTERPRÉTATIONS
}

\author{
Patrice LECOQ *
}

Localisé au cœur de la cordillère de Vilcabamba, au Pérou, à quelque $150 \mathrm{~km}$ au nord-ouest de Cuzco, l'ancienne capitale de l'État inca, Choqek'iraw est l'un des rares sites préhispaniques et le seul de l'époque inca à posséder une grande mosaïque murale. Plusieurs indices laissent penser que les motifs représentés ont une dimension cosmologique et que la façon dont ils sont agencés s'inspire de principes textiles, ce que cet article tentera de démontrer. Dans un premier temps, nous replacerons ce site dans son contexte géoculturel, en le décrivant brièvement. Dans un second temps, nous détaillerons les principales caractéristiques des décors pour tâcher d'en comprendre le sens. De fait, les thèmes représentés pourraient se fonder sur d'anciens mythes et renvoyer à des conceptions d'ordre astronomique. [Mots-clés: Pérou, Andes, Inca, Choqek'iraw, mosaïques, archéoastronomie, textile, pastoralisme.]

Themosaic-adorned terraces of the Inca site of Choqek'iraw, Peru. General description and some elements for their interpretation. Choqek'iraw, in the heart of the Vilcabamba cordillera, in Peru, some $150 \mathrm{~km}$ from the ancient Inca State capital Cuzco, is one of the very few Pre-Hispanic sites displaying large wall mosaics, and the only one known from Inca times. This paper will attempt to demonstrate that the figures represented on the walls convey cosmological significance and are laid out following textile principles. We will first describe the site and its geo-cultural setting. Then, the mosaics' main characteristics will be examined in detail, in attempt to grasp their meaning. We conclude this paper by suggesting that some of the scenes depicted might have been inspired by pre-Columbian myths and might be linked to astronomical concepts. [Key words: Peru, Andes, Inca, Choqek'iraw, mosaic, archaeoastronomy, textile, pastoralism.]

Los andenes con mosaicos del sitio inca de Choqek'iraw, Perú. Descripción general y elementos preliminares de interpretación. Ubicado en el corazón de la cordillera de

* Université de Paris 1/UMR 8096 (Archéologie des Amériques), Maison René-Ginouvès (Archéologie et Ethnologie), 21 allée de l'université, 92023 Nanterre [Patricelecoq@free.fr].

Journal de la Société des Américanistes, 2010,96-2, pp. 7-73. OSociété des Américanistes. 
Vilcabamba, a unos $150 \mathrm{~km}$ al noroeste de Cuzco, la antigua capital del estado inca, Choqek'iraw es uno de los pocos sitios prehispánicos, y el único de época inca, con un gran mosaico mural. Varios indicios nos hacen pensar que los motivos de estos mosaicos refieren a concepciones astronómicas y que la manera de representarlos reproduce patrones de los textiles, lo que este trabajo intentará demostrar. Asimismo, después de hacer una breve descripción del sitio, ubicándolo en su contexto geocultural, se analizarán las principales decoraciones que allí se encuentran para tratar de entenderlas. Los temas representados podrían referirse a antiguos mitos andinos y concepciones de orden astronómico. [Palabras claves : Perú, Andes, inca, Choqek'iraw, mosaicos, arqueoastronomía, textil, pastoralismo.]

À la mémoire d'Elisabeth Bonnier et d'Olivia Harris, qui m'initièrent à l'archéologie et à l'anthropologie des Andes et des Hauts Plateaux boliviens

\section{CHOQEK'IRAW, UN SITE CÉRÉMONIEL INCA}

Considéré comme l'un des sites majeurs de la culture inca, Choqek'iraw ${ }^{1}$ ou Choque Quirao (terme en langue quechua qui se traduit généralement par " berceau de l'or $~^{2}$ ) est localisé dans les Andes péruviennes, à la limite des départements de Cuzco et d'Apurimac, non loin du site de Sayhuite (Figure 1). Construit sur les crêtes et les versants abrupts de la montagne éponyme, à une altitude moyenne de $3200 \mathrm{~m}$, il comprend de nombreux vestiges architecturaux : terrasses, plates-formes, places cérémonielles, temples, entrepôts, fontaines et canaux, reliés par d'interminables escaliers et par tout un réseau de chemins (Figures 2 et 3). Le site est dominé au nord-est par le grand massif du Corihuayrachina ou Qoriwayrachina, aussi dénommé Yanacocha ou encore Marcani ${ }^{3}$. Il est entouré par de nombreux pics enneigés, tels les cerros Pumasillo et Choqetacarpo au nord, Wiracochan au nord-ouest, Ampay au sud et Salkantay à l'est, que les habitants des villages proches considèrent comme des lieux sacrés où résident les âmes des ancêtres, les apu (Reinhard 1983, 1985, 2002 ; Scanu 1987 ; Ceruti 1999). Compte tenu du peu de données publiées sur ce site et son contexte (Samanez et Zapata 1995, 1999; Lumbreras, Uccelli et Wust 2001 ; Lecoq et Duffait 2004 ; Lecoq 2004, 2005 ; Zapata 2005, 2006 ; Valencia García et al. 2005 ; Ravines 2006 ; Samanez 2006 ; Echevarría López 2008), une brève description générale s'impose. 


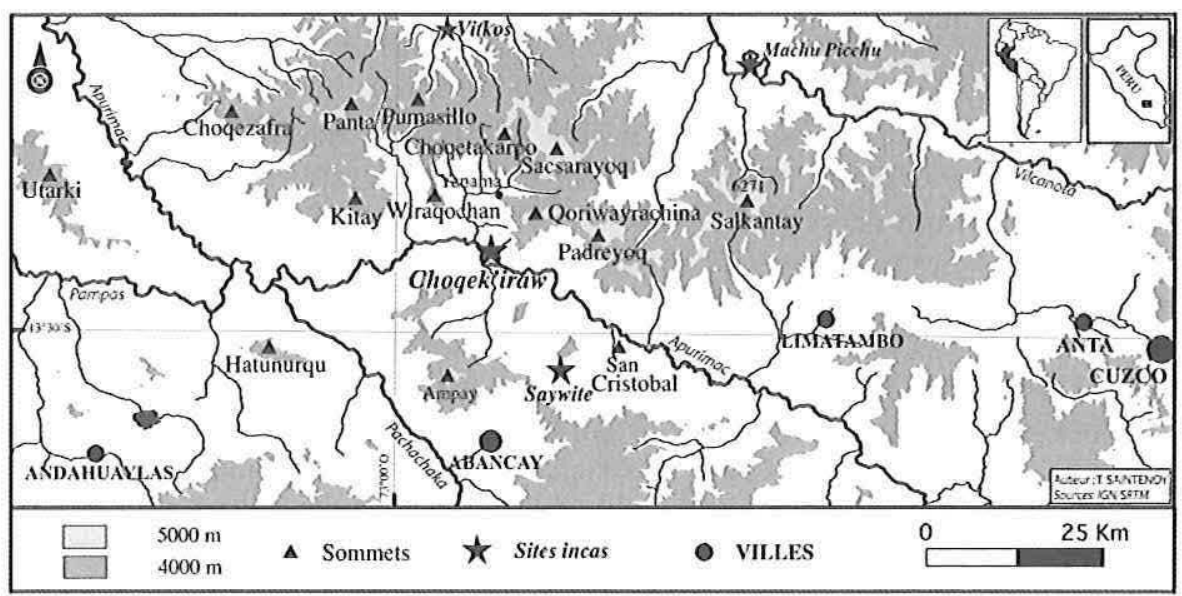

FIG. 1 - Localisation du site de Choqek'iraw.

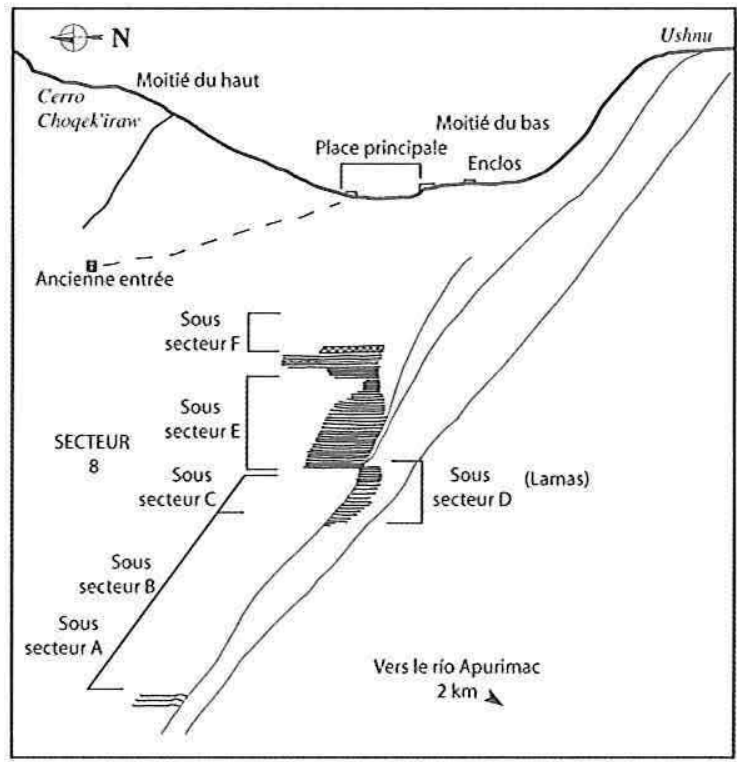

Fig. 2 - Vue en élévation simplifiée du versant occidental de Choq̨ek'iraw (en haut, ligne de crête et, en bas, S.8) montrant la localisation des terrasses décorées (redessiné d'après un cliché d'Echevarría López 2008, p. 67). 


\section{Description générale du site}

Les différents édifices de Choqek'iraw se distribuent entre plusieurs quartiers urbains et périphériques, aujourd'hui répartis en treize secteurs et plusieurs sous-secteurs, dont certains, toujours enfouis sous la végétation, n'ont pas été cartographiés. Les plus nombreux et les mieux préservés se situent dans le noyau urbain qui couvre près de 11 ha.

À l'instar de l'ancienne capitale des Incas, Cuzco, ou d'autres sites incas régionaux mieux connus comme Machu Picchu, Choqek'iraw semble avoir été organisé en deux moitiés (grisées sur la Figure 3) : l'hanan ou haut et l'hurin ou bas (Garcilaso de la Vega 1982 [1609], livre 1, chap. XVI ; Cobo 1964 [1653], tome 1, chap. VIII ; Bouysse-Cassagne 1978 ; D’Altroy 2005, pp. 87-121 ; Pärssinen 1992, pp. 171-192).

\section{Hanan, la moitié du haut (S.1 et S.2 sur la Figure 3)}

Ce secteur abrite divers monuments à l'architecture soignée, regroupés autour d'une place, selon l'organisation typique des cités incas (Gasparini et Margolies 1977). On y trouve au nord, une fontaine, alimentée par un canal de drainage qui descend de la montagne éponyme et traverse le site du nord au sud, ainsi qu'un édifice à deux niveaux, vraisemblablement lié au culte, désigné comme temple (hanan) sur la Figure $3{ }^{4}$. Plusieurs autres monuments sont construits plus au sud et orientés en fonction des points cardinaux et des principaux sommets. L'un d'entre eux, le « temple au rocher », intègre, dans son architecture, un gros rocher dont la forme évoque celle du Cerro Corihuayrachina, localisé au nord-est, mais d'autres rochers de ce type sont éparpillés à travers tout le site ${ }^{5}$.

Un peu plus au sud-est se dresse un complexe de greniers (collca ou colqa ; à l'est de S.2 sur la Figure 3), formé par un ensemble de cinq édifices, de plan rectangulaire et de forme allongée, dont les plus imposants s'étagent sur deux niveaux. Les fouilles qui y ont été menées par les archéologues de Copesco, sous la direction de Percy Paz, ont permis de déterminer que les pièces supérieures servaient à entreposer des denrées, comme le maïs, alors que les pièces inférieures étaient utilisées pour ventiler l'édifice (Zapata 2005, p. 115), selon un modèle comparable à celui observé, à moindre échelle, par Morris (1967) à Huanuco Pampa.

Seize courtes terrasses, alimentées par un canal et délimitées par deux petits murs latéraux, s'étagent plus au sud. Elles font face au Cerro San Cristobal $(3100 \mathrm{~m})^{6}$, localisé à quelque $24 \mathrm{~km}$ en amont, au sud-est, près de l'emplacement supposé du sanctuaire consacré à l'oracle de l'Apurimac (Cieza de Léon 1984 [1553], p. 256) ${ }^{7}$ et semblent descendre vers le fleuve qui coule en contrebas. Des petits escaliers, disposés en alternance sur les extrémités des terrasses, tous les deux niveaux, permettent d'accéder à chacune d'entre elles. Par ailleurs, à chaque 


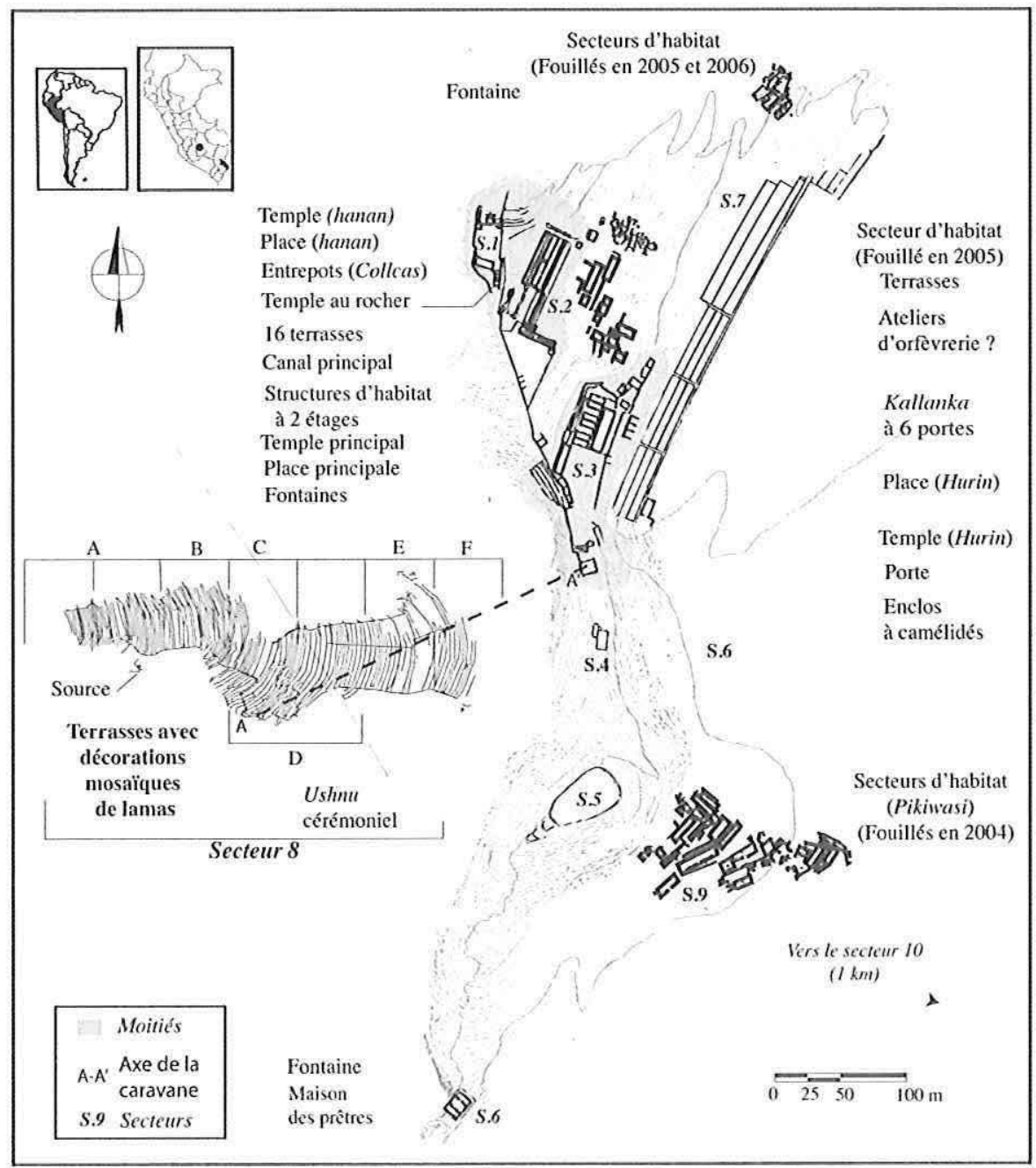

Fig. 3 - Carte générale de la partie centrale de Choqek'iraw actuellement (redessinée d'après le plan original établi par Copesco en 2003 et la carte d'Echevarría López 2008).

niveau de terrasse, une petite niche a été aménagée à l'intérieur des deux murs latéraux ${ }^{8}$. Enfin, de grosses pierres qui reproduisent la forme des montagnes environnantes comme le Cerro Corihuayrachina ont été intégrées dans les murs de contention. Un vaste secteur d'habitat, encore partiellement enfoui sous la végétation, est situé un peu plus à l'est. Il réunit diverses structures de plan rectangulaire, de type $k a l l a n k a^{9}$, érigées sur des terrasses exiguës et organisées 
autour de patios de plans irréguliers, qui auraient pu être utilisées comme temples ou salles de réunion lors de certaines cérémonies. On y trouve également des structures circulaires à caractère domestique (Lecoq 2008) ${ }^{10}$.

\section{Hurin, la moitié du bas (S.3 et S.4 sur la Figure 3)}

Localisée à quelques centaines de mètres plus au sud et plus en contrebas, la moitié du bas abrite les édifices les plus notables de Choqek'iraw, là encore agencés autour d'une place. On dénote, tout d'abord, au nord, trois grandes bâtisses de plan rectangulaire, à deux niveaux, disposées de façon symétrique et accessibles par une porte à double jambage ; l'espace intérieur s'organise autour de patios. Certains pans de murs conservent encore les pignons servant à supporter la toiture dont le degré d'inclinaison reprend celui du Cerro Corihuayrachina visible en arrière-plan. Ces édifices étaient probablement les demeures des membres de l'élite locale. Juste à côté, à l'est, se dresse un vaste bâtiment, de type kallanka, de plan rectangulaire et d'un seul niveau, de $33 \times 7,5 \mathrm{~m}$, muni de six portes ouvrant sur le levant (Samanez 2006, p. 98). Il renferme plusieurs niches basses, hautes de $1,60 \mathrm{~m}$. Chaque niche est encadrée par de petits anneaux de pierres fichés dans les murs adjacents. Zapata (2006, p. 104) suggère que ces niches auraient pu renfermer des idoles ou les copies des corps momifiés de personnages de haut rang, lors de certaines fêtes, dissimulés par une pièce d'étoffe accrochée aux anneaux latéraux cités. La partie au nord de ce secteur abrite des petites bâtisses et des cours qui pourraient avoir été utilisées comme ateliers d'orfèvrerie, à en croire les vestiges (objets en cuivre et outillage de pierre pour concasser les minerais) mis au jour lors des fouilles.

Un vaste édifice rectangulaire, de forme allongée, là encore de type kallanka, mais sans aucune décoration interne, identifié comme temple principal, occupe l'extrémité sud-occidentale de la place. On y accède par quatre grandes portes situées à l'est. Il laisse place, au sud, à une petite construction renfermant deux fontaines, alimentées par le canal provenant de la moitié du haut.

Un autre édifice, de plan rectangulaire, désigné cette fois comme temple (hurin), qui présentait autrefois deux niveaux, occupe l'extrémité méridionale de la place. Sa façade septentrionale est pourvue de quatre portes trapézoïdales à double jambage caractéristiques de l'architecture cérémonielle inca. La perfection de son architecture lui a valu d'être qualifié par Angrand (1972), de « mur triomphal », lors de sa visite du site au $\mathrm{XIX}^{\mathrm{e}}$ siècle. Chaque porte, ouvrant au nord, donne vers un élément du relief environnant. Certains détails architecturaux de cet édifice laissent penser que l'accès au niveau supérieur s'effectuait par l'intermédiaire d'une plate-forme située au-dessus. Une porte, localisée dans le prolongement oriental du mur de façade, contrôlait l'accès au secteur méridional, mais restreignait également l'entrée à la place du bas. Dix-sept contextes funéraires ont été trouvés dans ce secteur. Il s'agit d'enterrements primaires, aménagés dans des puits et protégés par des rangées de pierre (Zapata 2005, p. 107). 


\section{Les secteurs méridionaux (S.4, S.5 et S.6)}

Ces secteurs regroupent plusieurs bâtiments à caractère rituel, administratif et domestique, ainsi que deux enclos qui étaient probablement utilisés pour garder les animaux assurant le ravitaillement du site ou ceux destinés à être sacrifiés lors de certaines cérémonies, selon Paz Flores (communication personnelle), comme c'était le cas pour de nombreux établissements incas régionaux à caractère cérémoniel (Bauer 2000, p. 27). On y trouve également une colline tronquée, définie comme une plate-forme cérémonielle ou ushmu ${ }^{11}$ par les archéologues de Copesco qui ont établi le plan du site. À Choqek'iraw, le terme ushmu ne désigne pas une plate-forme rituelle artificielle comme c'est le cas pour d'autres sites incas tels que Wilcaswaman, Curampa ou Huanuco Pampa (Gasparini et Margolies 1977, pp. 204-228), mais un grand monticule naturel au sommet aplani, vraisemblablement cérémoniel, de plan plus ou moins circulaire et entouré par un petit parapet interrompu au nord-est (Figures 3 et 4). À ce promontoire naturel qui fut intentionnellement modifié, on accède, depuis la place du bas (et depuis le S.3), en franchissant une porte, à double jambage, aménagée sur l'un des côtés du temple du bas. Certains indices, comme la localisation de cette plate-forme sur un promontoire dominant l'intégralité de la vallée Apurimac et permettant d'observer la totalité des montagnes environnantes, ainsi que les vestiges trouvés à son sommet, laissent penser qu'elle aurait pu faire office d'observatoire et de lieu de culte à la fois (Lecoq 2007) ${ }^{12}$.

La partie la plus lointaine du versant méridional de Choqek'iraw (S.6) comprend une fontaine, ainsi que deux bâtiments à deux étages, placés en vis-à-vis, suivant un groupement " par opposition » selon la nomenclature de Bouchard (1983), qui ont été désignés comme les maisons des prêtres en raison de leur localisation et de leur orientation très particulières. Chaque édifice comporte, à l'est et à l'ouest, trois niches trapézoïdales superposées, deux en bas, fermées, et une ouverte en haut. L'ouverture placée du côté occidental est orientée vers les trois pics du Cerro Wiracochan localisé à $11 \mathrm{~km}$ et à $310^{\circ}$ au nord-ouest du site ${ }^{13}$, et les portes septentrionales vers la cime du Cerro Corihuayrachina.

Le moyen versant oriental de la montagne (S.9), aussi dénommé Pikiwasi ou «La maison de la puce » ou, encore, " secteur des maisons populaires », correspond à un groupe de structures, de plans rectangulaire et circulaire, érigées sur d'étroites terrasses - que nous avons partiellement fouillées - séparées par des ruelles étroites, des passages parallèles, d'où partent de petits escaliers. Les nombreuses fusaïoles exhumées par les archéologues de Copesco lors des fouilles de certaines structures rectangulaires, aujourd'hui restaurées, les ont conduits à dire qu'il pourrait avoir existé là des ateliers de tissage ${ }^{14}$. 


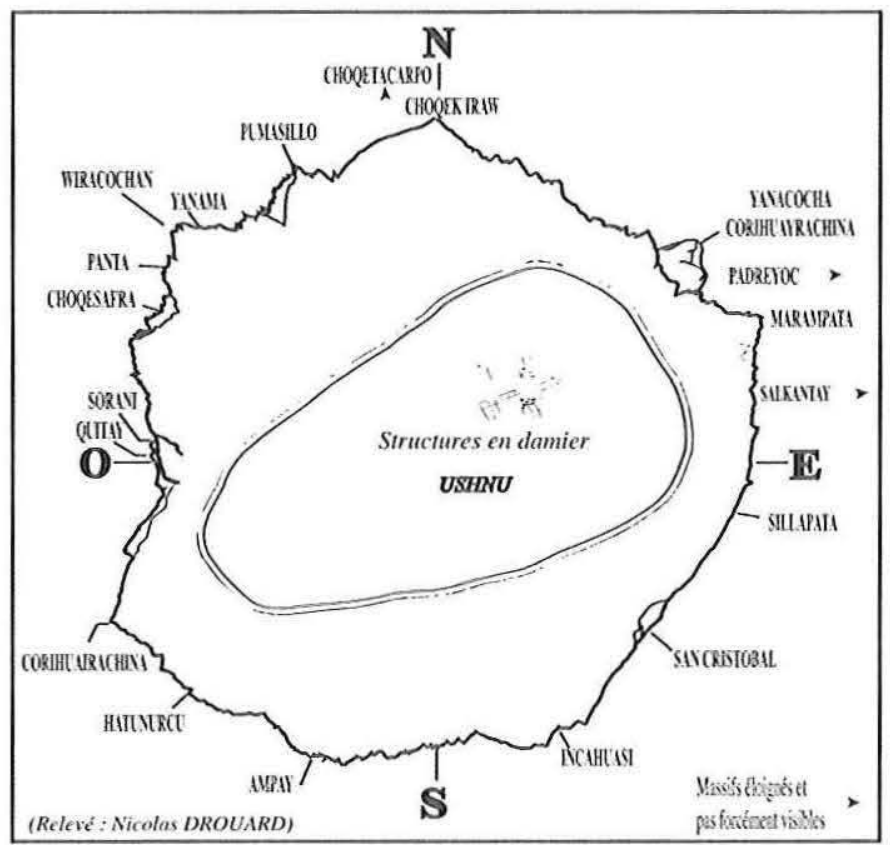

Fig. 4 - Croquis des principaux sommets visibles depuis l'ushnu et les structures en damier récemment fouillées.

\section{Les terrasses des versants orientaux et occidentaux}

De multiples terrasses, à usage vraisemblablement agricole, occupent les deux versants de l'éminence de Choqek'iraw.

Les plus spectaculaires, car visibles de loin, sont celles du versant oriental, regroupées en quatre ensembles, s'étendant sur trois niveaux, qui confèrent au site son identité (S.7). Chaque terrasse est limitée en aval et en amont par un mur de soutènement d'un appareillage soigné. Trois escaliers permettent de passer d'une terrasse à une autre. Des dalles insérées dans le mur font aussi office de marches. En contrebas, s'étend un vaste complexe de terrasses (non représenté sur le plan d'ensemble du site ${ }^{15}$ ) défini comme S.10 et désigné sous le terme de phaqcha wayq'o "la cascade " ${ }^{16}$, en référence à la chute d'eau localisée au nord-est. Celui-ci regroupe quelque quatre-vingts terrasses, réparties en six colonnes séparées par de petits canaux d'irrigation et des escaliers fichés dans le mur de soutènement. Au centre se dresse une bâtisse d'une pièce, à deux niveaux, désignée comme " La maison de la chute d'eau » ou celle du chaqra kamayoc (« le responsable des cultures »). La partie supérieure de certains des murs est décorée 
de petites corniches, constituées de dalles de pierres saillantes qui pourraient être d'inspiration chachapoya (Lumbreras 2005). Samanez (2006, p. 97) suggère que ces corniches évitaient le ruissellement des eaux sur les murs, protégeant ainsi le crépis et les peintures qui les recouvraient. Une fontaine y a également été construite. Enfin, plus en contrebas, au sud-ouest, se trouve un gros rocher aplani, de couleur blanche, orienté du nord-est au sud-ouest, qui pointe vers l'Apu Ampay, localisé de l'autre côté de la vallée.

Sur le versant occidental du site, le S.8 compte quelque cent vingt-neuf terrasses exiguës, dont vingt-cinq ornées de mosaïques représentant vingt-huit motifs. Elles mettent en scène des camélidés, une figure anthropomorphe et des dessins géométriques (Figures 5, 6, 7 et 8. Valencia García 2004 ; Valencia García et al. 2005 ; Echevarría López 2008) sur lesquels nous reviendrons en détail. On y trouve également l'ancienne voie d'accès au site (découverte en août 2005) et la porte ${ }^{17}$ qui permettait d'y pénétrer. En la franchissant, le visiteur ou le pèlerin en route vers Choqek'iraw apercevait, au détour du chemin, les grands panneaux emblématiques de ce lieu vraisemblablement cérémoniel.

Plusieurs autres secteurs de terrasses se répartissent autour de l'agglomération centrale. Les plus notables sont celles de Paraqte pata placées en amont et celles de Pincha unuyuc localisées à 7,6 km au nord-ouest du site principal, sur la route de Huancacalle et de Yanama.

\section{Quelques témoignages historiques relatifs à Choqek'iraw}

Plusieurs sources ethnohistoriques font mention de Choqek'iraw et montrent que ce site était connu des Espagnols dès leur arrivée au Pérou, au $\mathrm{xvI}^{\mathrm{e}}$ siècle. Duffait $(2005 ; 2007$, pp. 214-226) indique qu'il pourrait s'agir d'un domaine royal privé de l'Inca Tupac Yupanqui. Les travaux de Samanez et Zapata (1995; 1999) et un ouvrage récent (Echevarría López 2008, pp. 46-48) suggèrent que le site a été planifié sous le règne de Pachacutec et agrandi par Tupac Yupanqui. C'est de cette époque que dateraient les mosaïques qui y ont été découvertes. Le fait que Choqek'iraw ait été effectivement un domaine royal pourrait expliquer le caractère exceptionnel des terrasses et des édifices érigés à Choqek'iraw. Comme d'autres manifestations matérielles incas (céramiques, textiles, orfèvrerie...), ces édifices servaient à magnifier la puissance du souverain de son vivant (Bray 2008), à perpétuer sa mémoire après sa mort et à subvenir aux besoins des membres de son lignage ou panaca (Duffait 2007, p. 2).

Tout au long du XIX ${ }^{\mathrm{e}}$ siècle, plusieurs explorateurs, parmi lesquels Eugène de Sartiges (1851; 2006), Léonce Angrand (1972) et Charles Wiener (1993 [1880]) ont entrepris la visite du site ou l'ont mentionné dans leurs travaux. Samanez (2006) et Ravines (2006) en font une synthèse dans un numéro spécial du Boletín de Lima. En 1909, Bingham (1910 ; 2006), pour sa part, a effectué des fouilles, notamment au sommet de la colline tronquée désignée comme ushmu. 
Vers les terrasses supérieures

décorées de motifs en zig-zag et en damiers ( $82 \mathrm{~m}$ )

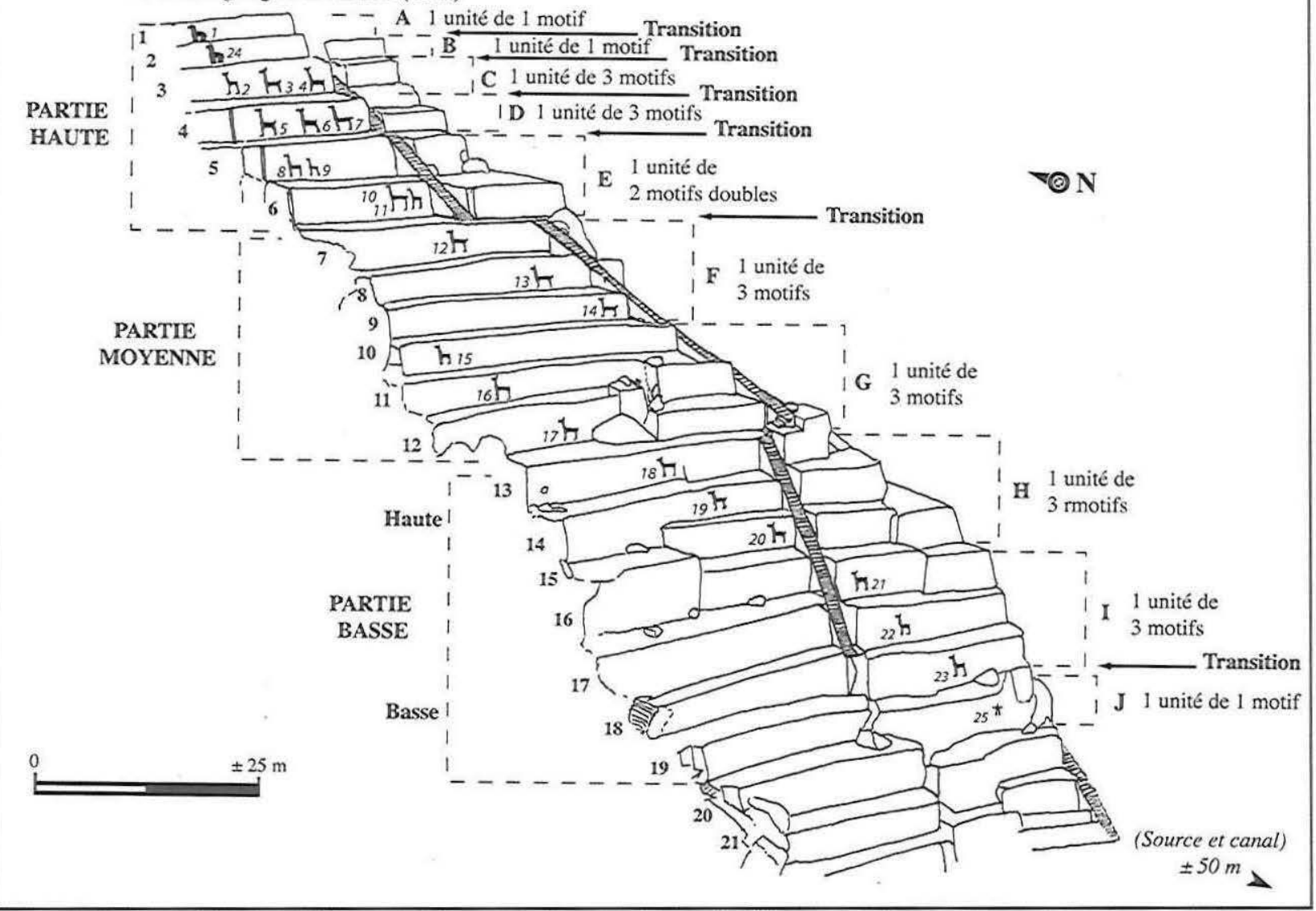

Fig. 5 - Vue d'ensemble des mosaïques du versant occidental, S.8, sous-secteur D. de Choqek iraw. 


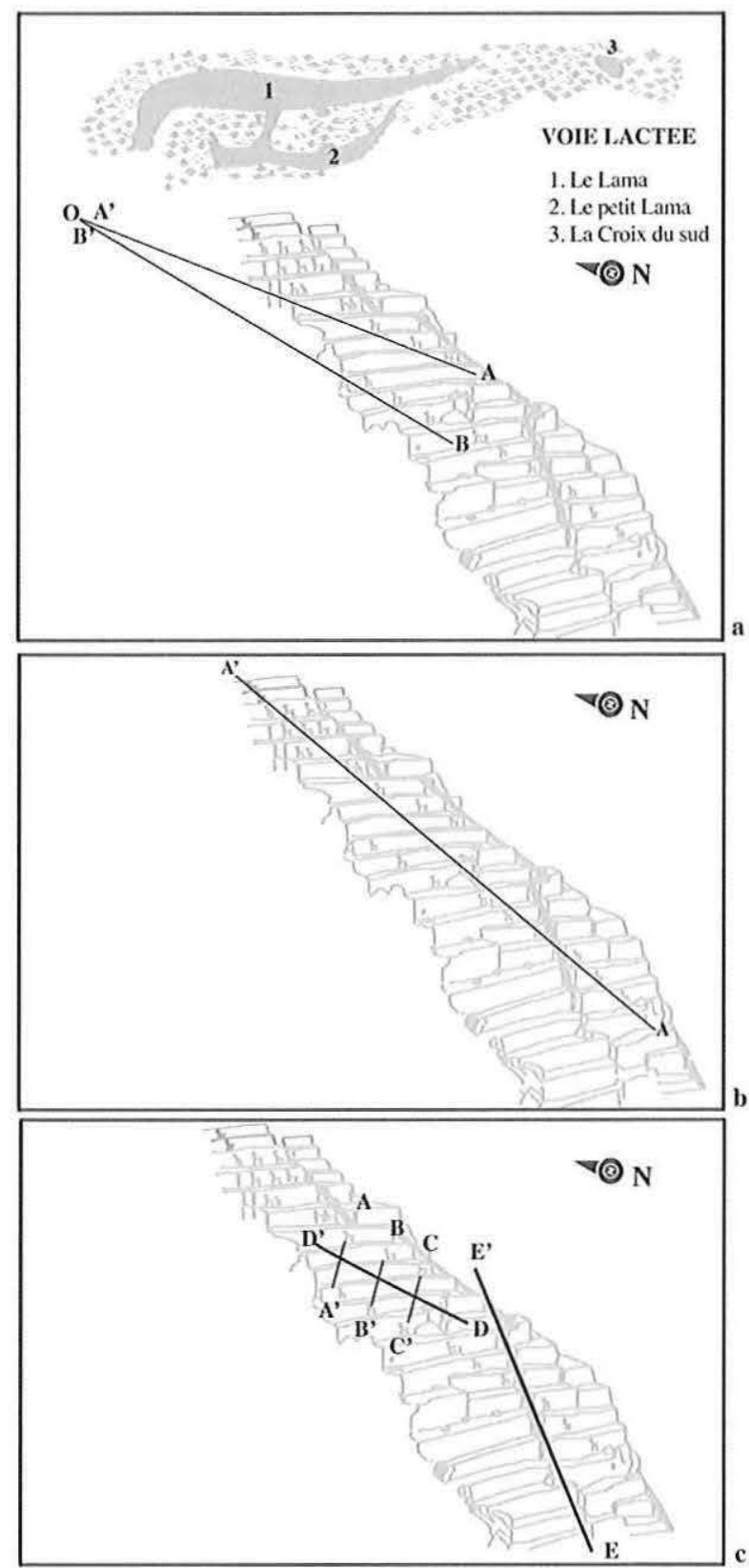

FIG. 6 - Principales lignes de fuite des différents motifs de lamas représentés sur les mosaĩques du versant occidental de Choqek'iraw, avec l'emplacement de la Voie Lactée en juin. 


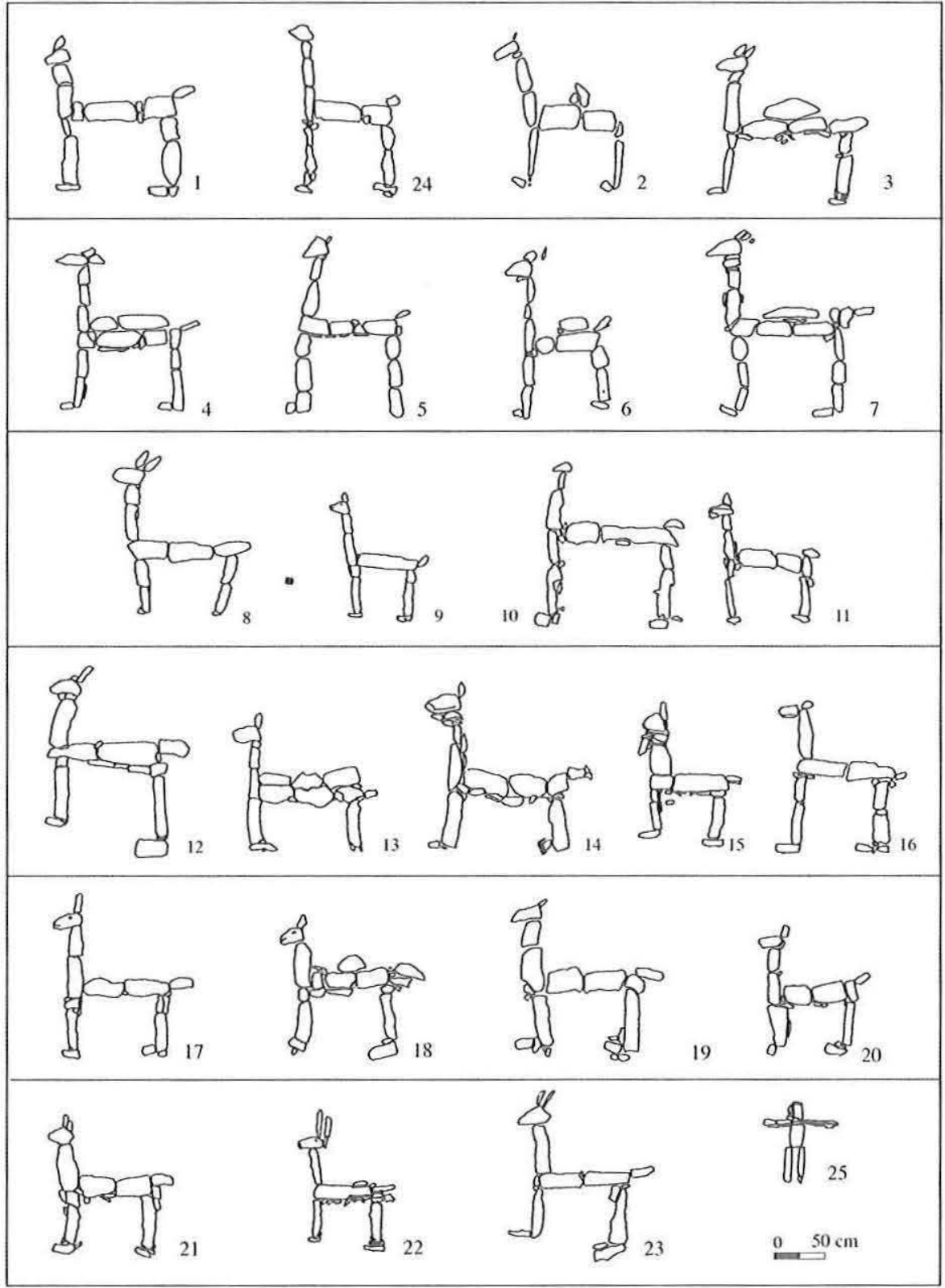

FIG. 7 - Principales figures, à la même échelle, représentées sur les terrasses occidentales du S.8, sous-secteur $\mathrm{D}$, de Choqek'iraw (dessins réalisés à partir de diapositives et de clichés numériques; les numéros reprennent la nomenclature de Copesco, présentée dans Echevarria López 2008). 


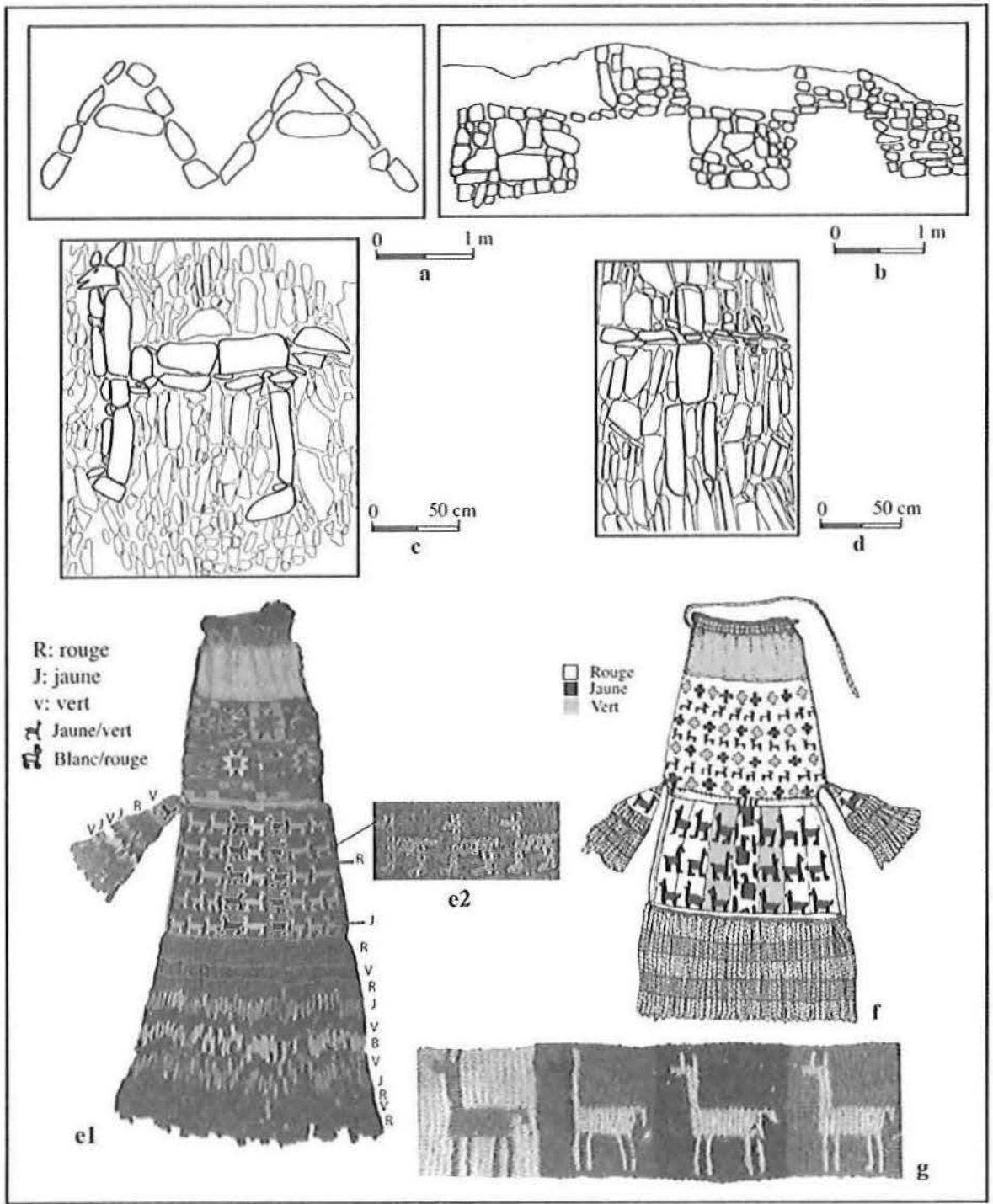

FIG. 8-Motifs d'inspiration textile représentés sur les terrasses de Choqek'iraw :

a et b : relevés schématiques des motifs en damier et en zigzag des terrasses supérieures proches de la place centrale de Choqek'iraw.

c et d: détails des motifs de lamas et de la figure anthropomorphe (terrasses médianes et inférieures), montrant la disposition des différentes pierres formant les décors qui évoquent les fils de chaîne d'un textile.

el et e2 : sac à coca, culture inca. Coton et laine, tapisserie et broderie. $43 \times 25 \mathrm{~cm}$, frange : $28 \mathrm{~cm}$ (musée national d'Archéologie et d'Anthropologie, Pueblo Libre, Lima, modifié d'après photo).

$f$ : sac à coca, culture inca. Coton et laine, tapisserie et broderie ; lamas et cervidés de couleur jaune sur rouge et jaune sur vert (musée régional d'Ica, redessiné d'après photo).

$\mathrm{g}$ : fragment d'une ceinture actuelle de l'Altiplano bolivien, tissée selon une technique de trame enroulée (cliché de Desrosiers). 
Aujourd'hui, les fouilles et les nombreux travaux de restauration qui ont été réalisés à Choqek'iraw par les archéologues de Copesco - grâce à un financement émanant du Fond Contre-valeur : Pérou-France - apportent un éclairage nouveau sur l'histoire du site (Alencastre Montufar 2006 ; Samanez et Zapata 1995, 1999). Ainsi, les fouilles que nous avons nous-mêmes effectuées, dans deux secteurs d'habitat domestique, localisés respectivement à l'est de l'ushmu et au nord des grandes terrasses (Figure 3), ont révélé une occupation plus ancienne que celle connue jusque-là. Celle-ci pourrait remonter à la fin de l'Horizon moyen, vers $1000 \mathrm{apr}$. J.-C., et s'être fortement accentuée durant l'Intermédiaire récent (1000-1300), sans parler des traces d'une possible occupation au cours de l'Intermédiaire ancien, entre 200 av. et 500 apr. J.-C. (Lecoq 2004 ; 2008).

\section{Des Choqek'iraw multiples}

Comme l'ont fait remarquer Zuidema (1974-1976, pp. $212-214$; 1978) et Bauer (2000), Choquequirao ou Chuquiquirao est le nom donné à une montagne sacrée (huaca ou wak'a) de la région nord-est de Cuzco, dans l'Antisuyu, d'où jaillissait une source appelée Chuquiquirao puquio, à laquelle on rendait hommage en sacrifiant des lamas et des tissus. Ce huaca était placé sur le quatrième ceque de l'Antisuyu, selon le système qui caractérisait l'organisation sociopolitique et rituelle de Cuzco, dans les données transmises par Cobo et Polo de Ondegardo ${ }^{18}$. Il est donc possible que, comme pour d'autres centres cérémoniels de la région de Cuzco, récemment révisés par Bauer (2000, p. 178), le site de Choqek'iraw - si tel était effectivement son nom à la période inca - ait été conçu comme une projection plus ou moins idéalisée de ce huaca dans la vallée de l'Apurimac, lié au culte solaire et à l'Inca, ce que les données archéologiques ne permettent toutefois pas de vérifier ${ }^{19}$.

\section{DES MOSAÏQUES DE PIERRE COMIIE EXPRESSION DE L'ART MURAL ANDIN}

Les mosaïques de pierre retrouvées à Choqek'iraw appartiennent à une longue tradition d'art mural qui remonte à la Période initiale (1800-1500/900 av. J.-C.). Elle a beaucoup évolué jusqu'à la période inca (Horizon récent : 1440-1539 apr. J.-C.) et on peut dire qu'elle s'est poursuivie jusqu'à nos jours. Comme l'a montré Bonavia (1974; 1990a et b, p. 156), cet art a pris la forme, le plus souvent, « de peintures murales, réalisées sur des murs de terre, qui diffèrent des peintures rupestres exécutées sur les parois des grottes et abris. D'ailleurs, le fait de peindre des édifices est une coutume beaucoup plus généralisée que ce que l'on pense et l'on ignore encore si cette pratique obéit à des schémas culturels spécifiques et déterminés par certaines normes ou si elle est le fruit d'impulsions esthétiques innées et personnelles de l'homme andin » (Bonavia 1974, p. 157). 
Toujours selon cet auteur, "cette expression artistique est étroitement associée aux pratiques religieuses et s'est surtout manifestée dans la décoration des temples et des palais où elle était liée aux croyances des différentes cultures qui se sont développées tout au long de l'époque préhispanique " (Bonavia 1990b, p. 155). Les peintures étaient donc un aspect très important, voire essentiel, du rituel magico-religieux de l'ancien Pérou et Choquek'iraw n'échappe pas à cette règle. Pour Bonavia $(1974$, p. 145), «la peinture murale ne fut toutefois pas l'unique moyen employé pour orner les façades des édifices préhispaniques ; deux autres matériaux étaient largement utilisés : l'or et les tissus. Dans de nombreux cas, les motifs peints s'inspiraient d'ailleurs de motifs textiles, dont ils pouvaient aussi être la copie plus ou moins fidèle. Il est probable que des toiles peintes et des tapis aient été utilisés comme décorations murales avant d'être finalement remplacés, au cours des périodes tardives, par des peintures plus ou moins similaires ». Les mosaïques murales semblent avoir été peu répandues ou n'ont pas laissé de traces, et les quelques exemplaires recensés sont tardifs. On les trouve principalement dans des sites de la culture chachapoya de Kuelap et de l'Abiseo (Bonavia 1990c), au nord du Pérou, datés des périodes Intermédiaire récente ( $\mathrm{XII}^{\mathrm{e}}-\mathrm{XIV}^{\mathrm{e}}$ siècles) et Inca, donc contemporains de Choqek'iraw (Schjellerup 1990 ; Kauffmann Doig 2002) ${ }^{20}$.

\section{Histoire de la découverte des mosaïques}

Les premières mosaïques de Choqek'iraw ont été découvertes par l'archéologue Paz Flores en 2002, mais elles n'ont été rendues publiques qu'en septembre 2004, à l'occasion d'un colloque organisé à Cuzco (Paz Flores 2007). Par la suite, plusieurs autres mosaïques décorées de motifs géométriques, anthropomorphes et zoomorphes ont été mises au jour, notamment par l'archéologue péruvien Valencia García, lors du nettoyage des hauts versants du S.8 (soussecteur D), puis restaurées. Les terrasses qui les portent se situent en contrebas de la place centrale de la moitié du bas, en dessous de l'édifice défini comme temple (hurin) et des fontaines construites à proximité (Figure 3). À leur emplacement se trouve une petite source plus ou moins asséchée ; elles s'étagent entre $2766 \mathrm{~m}$ et $3010 \mathrm{~m}$ d'altitude et couvrent près de $185700 \mathrm{~m}^{2}$. On y accède depuis l'angle nord-ouest de la place du bas, par un petit chemin abrupt qui serpente dans la montagne. À la période inca, l'accès se faisait aussi probablement depuis l'entrée principale du site, non représentée sur la Figure 3. Chaque terrasse restaurée mesure près de $80 \mathrm{~m}$ de long sur $1,50 \mathrm{~m}$ de large, mais seule la partie actuellement dégagée est effectivement décorée. C'est en fait tout le versant occidental de Choqek'iraw qui était donc recouvert de terrasses, dont l'organisation et le symbolisme évoquent les terrasses de Yucay, dans la région de Cuzco, étudiées par Molinié (1996). 


\section{Organisation et agencement des motifs}

Ces mosaïques se composent de vingt-huit motifs en pierre, correspondant à cinq types : des lignes, des lignes brisées, des damiers, des lamas stylisés et un personnage anthropomorphe (Echeverría López 2008). Les lamas et le personnage sont disposés sur dix-neuf terrasses abruptes, orientées vers les trois cimes du Cerro Wiracochan, déjà mentionné. Ce massif, localisé au nord-ouest, semble avoir joué un rôle déterminant à Chogek'iraw, peut-être en lien avec les mouvements du soleil, lors des solstices ou des équinoxes, avec ceux de la lune ou avec l'apparition de certaines constellations (comme la Voie Lactée et les Pléiades) à des moments précis de l'année. Il conviendrait de faire une recherche sur ce sujet. Cette orientation des motifs vers le soleil couchant est à l'origine du nom évocateur, mais un peu arbitraire, qui leur a été donné par Zapata (2005; 2006), « les lamas du Soleil », cette appellation ne reposant sur aucun témoignage historique. L'ensemble constitue un grand panneau mural, visible dans son intégralité seulement de loin. Il surplombe la rivière Apurimac qui coule en contrebas, dans un profond canyon, vers l'ouest, avant de gagner le piémont amazonien.

Pour faciliter l'étude de ces mosaïques, les archéologues de Copesco ont divisé le versant en six sous-secteurs, indiqués de $\mathrm{A}$ à $\mathrm{F}$ sur les Figures 2 et 3. Deux d'entre eux, les sous-secteurs $\mathrm{D}$ et $\mathrm{F}$, présentent des décorations, dûment répertoriées et numérotées de 1 à 28 (Echevarría López 2008). Nous avons repris cette nomenclature sur les relevés de terrain et sur les photographies réalisés entre 2004 et 2009. Ces sous-secteurs se situent respectivement sur la partie supérieure et moyenne du versant occidental et forment deux ensembles distincts, mais complémentaires.

Le premier sous-secteur, le F, correspond aux terrasses supérieures, relativement proches de la place centrale de Choqek'iraw, qui sont encore partiellement dissimulées sous la végétation, mais sont en cours de dégagement. Les motifs décoratifs y sont essentiellement linéaires et géométriques. Il s'agit, sur une première terrasse, située à $2982 \mathrm{~m}$ d'altitude, d'une ligne à zigzags (motif 26 selon la nomenclature adoptée par Echeverría López 2005, p. 209 ; 2008) formée de douze unités, pointe en haut et parfois renforcée par des segments horizontaux, placés juste sous le sommet (Figure 8a) ${ }^{21}$. Comme une grande partie du panneau est encore enfouie sous la terre, il est probable que d'autres motifs seront ultérieurement découverts. Chaque motif est formé par des pierres de calcschiste blanchâtre, disposées verticalement ou horizontalement. Elles se détachent des autres pierres, légèrement plus petites, placées à l'horizontal, qui forment le parement externe de la terrasse dont la hauteur fluctue entre 1,80 et $2 \mathrm{~m}$.

Une seconde terrasse, située à quelque $10 \mathrm{~m}$ en contrebas, dont la forme irrégulière et ondulée suit le versant accidenté de la montagne, présente un ensemble de douze damiers (Figure 8b) qui se prolonge sous les éboulements de 
terre. Ces motifs laissent place, à quelques mètres de là, à un dessin plus complexe, composé d'une série de lignes plus ou moins ondulées, sortant d'une sorte de grand rectangle évoquant ce qui pourrait être un autel ou un ushmu (non représenté), en cours de restauration.

Le second sous-secteur ( $D$, dans la nomenclature de Copesco) correspond aux terrasses inférieures. Localisées à environ $2900 \mathrm{~m}$ d'altitude - soit quelque $82 \mathrm{~m}$ en contrebas, pour une pente moyenne de $52^{\circ}$ d'inclinaison-, ces terrasses mettent en scène un ensemble de vingt-quatre lamas, de tailles et d'attitudes variées (Figures 7 et $8 \mathrm{c}$ ) accompagnés d'un petit persomnage, représenté les bras en croix, comme s'il embrassait l'univers (Figure 7, n 25 ; Figure 8d). Ces camélidés s'étalent sur une hauteur de $38 \mathrm{~m}$, d'après les relevés réalisés à l'aide d'un GPS $^{22}$. Ils sont figurés de profil, se dirigeant vers la gauche et vers le nord, pour un observateur placé devant le panneau. Chaque animal est formé par des dalles de calcschiste blanchâtre agencées de telle façon qu'elles en dessinent la tête, le corps et les membres. Dans certains cas, une incision esquisse l'œil et la bouche (Figure 7, $\mathrm{n}^{\circ}$ 9, 17, 18 ; Figure 8c). Chose curieuse, les pierres formant le parement de chaque terrasse sont disposées verticalement et non horizontalement comme c'est généralement le cas (Figure 8c et d). Quelques autres édifices et murs de terrasses, dispersés à travers le site, notamment dans le S.9 que nous avons fouillé, reprennent ce même mode de construction ou, si l'on préfère, de décoration qui reste une exception dans l'architecture inca.

En règle générale, chaque animal mesure $1,70 \mathrm{~m}$ de haut, depuis la partie basse de ses pattes avant jusqu'au sommet de ses oreilles, et 1,30 m de long, depuis l'avant de son poitrail jusqu'à sa queue, mais certaines figures sont plus petites. Le motif apparaît verticalement au centre du mur de terrasse, à $15 \mathrm{~cm}$ du sol, et à environ $30 \mathrm{~cm}$ du sommet. Les petits lamas disposés sur la partie haute du sous-secteur (Figure $5, \mathrm{n}^{\circ} 9,11$ ) mesurent $1 \mathrm{~m}$ de haut pour $0,75 \mathrm{~m}$ de long (Paz Flores 2007). Ces camélidés sont souvent associés à trois petites marches (non reproduites sur la Figure 5), formées par des dallées insérées dans le parement du mur de chaque terrasse, placées à près d'un mètre sur leur gauche. Vues de loin, ces marches accentuent l'effet ascendant de la caravane vers le nord-est. Elles permettent également de se rendre directement d'une terrasse à une autre sans emprunter les escaliers disposés sur le côté méridional des terrasses.

D'un point de vue stylistique, on pourrait répartir ces animaux en trois groupes :

- ceux, relativement stylisés - les plus nombreux - dont les différentes parties du corps sont constituées d'une seule dalle de pierre (Figure 7, $\mathrm{n}^{\circ}$ 1, 2, 8, 9, 11, 16, $17,19,23,31)$;

- ceux dont le corps est fait de deux ou plusieurs dalles placées l'une à côté de l'autre (Figure $7, \mathrm{n}^{\circ} 5,12,13,18$ ) ;

- ceux, plus complexes, constitués de tout un ensemble de dalles, y compris un renforcement du corps par plusieurs dalles superposées (Figure 7, $n^{\circ} 4,14,15,20$, 
22). Malheureusement, la restauration de ces figures leur a fait perdre certaines de leurs caractéristiques ${ }^{23}$.

Valencia García, qui a réalisé une analyse stylistique minutieuse de ces animaux en les comparant avec ceux, de même type, représentés sur des peintures rupestres du site de Negruyoc, localisé dans les environs de Yanama, sur le chemin menant à Vitcos ou Machu Pichu (Figure 1) y reconnaît des jeunes lamas, des petits avec leur mère, des lamas chargés de ballots et $\mathrm{y}$ voit même des scènes de copulation (Valencia García 2004 ; Valencia García et al. 2005). Paz Flores (2007) et Echevevarría López (2008) reprennent en partie ces interprétations et soulignent que ces animaux sont tous différents. Certains se tiennent bien droits, le poitrail légèrement bombé. D'autres se penchent en avant, alors que d'autres semblent marcher lentement, la queue repliée vers le bas ou dressée vers le haut, comme ils le font lorsqu'ils se déplacent en caravane (West 1979; Lecoq 1987; Flores Ochoa et al. 1995). Ils peuvent avoir les oreilles redressées ou tournées vers l'avant, comme s'ils étaient à l'écoute du pasteur qui les guide, ou repliées vers l'arrière (Figure 7, $n^{\circ} 3,4$ ). Enfin, si l'un porte un collier duquel semble pendre une cloche de bronze (Figure 7, $\mathrm{n}^{\circ} 15$ ), d'autres chargent des fardeaux plus ou moins volumineux (Figure $7, \mathrm{n}^{\circ} 3,4,6,7,13,18$ ) ${ }^{24}$.

Ces différentes caractéristiques, déjà observées par Valencia García (2004), laissent donc penser qu'il s'agit de la représentation d'une ou de deux troupes de camélidés en route vers les enclos proches de la place centrale de Choqek'iraw ou les pâturages d'altitude localisés près des communautés de Yamana, à un ou deux jours de marche du site, au pied du Cerro Corihuayrachina, où se trouvent des vestiges archéologiques à caractère pastoral (Saintenoy 2008). Ce massif semble avoir joué un rôle déterminant à Choqek'iraw, peut-être parce qu'il est le glacier proche du site où était prélevée l'eau servant à l'alimentation des fontaines. C'est aussi le lieu où semble se lever le soleil (Valencia García 2004) au moment du solstice de juin (Saintenoy communication personnelle, septembre 2009). De nos jours, les voyages de ce type ont lieu durant la saison sèche, de mai à septembre, au moment où les chemins sont secs et praticables. C'est aussi l'époque des grandes caravanes de troc entre les hauts plateaux et les vallées orientales des Andes, peu après la saison des pluies et la reproduction des camélidés (Custred 1974 ; Flores Ochoa 1974-1976, 1975 ; Flores Ochoa et al. 1995 ; West 1979 ; Merlino et Rabey 1983 ; Brougère 1984, 1988 ; Lecoq 1987 ; Browman 1988).

L'étude détaillée de chaque lama et de son emplacement au sein de la troupe, ainsi que la reproduction de tous à la même échelle, montre qu'il s'agit en fait de deux caravanes distinctes et d'animaux plus épars, de différentes tailles et, donc, peut-être d'âges divers. La première, sur les terrasses inférieures, guidée par le lama $\mathrm{n}^{\circ} 15$, comprend neuf animaux dont un chargé, le $\mathrm{n}^{\circ} 18$. La seconde n'en comprend que trois, non chargés, alors que les terrasses de la partie haute regroupent deux lamas femelles, accompagnées de leurs petits (Figure $5, n^{\circ} 5,6$ ) ainsi que des grands lamas - visiblement des mâles - dont quatre $\left(n^{\circ} 3,4,6,7\right)$ 
portent des charges. Les artistes qui ont décoré ces dernières terrasses avaient visiblement d'autres objectifs que celui de représenter une caravane en route, et ils ont vraisemblablement exprimé d'autres messages, peut-être liés à la fertilité des animaux, comme c'est souvent le cas dans l'art rupestre les mettant en scène (Flores Ochoa et al. 1995, pp. 37-44 ; Berenguer 1999, pp. 34-39). Peut-être s'agit-il de la transhumance d'une petite troupe de jeunes lamas vers les pâturages d'altitude, peu avant la saison sèche, de décembre à mars, comme cela est encore le cas aujourd'hui dans les communautés pastorales des hauts plateaux des Andes centrales. Si tel est le cas, les mosaïques de Choqek'iraw pourraient peut-être faire allusion à cette époque spécifique de l'année.

Une observation plus approfondie de la répartition des animaux sur ces terrasses nous permet également de déceler plusieurs détails révélateurs d'une certaine organisation de l'espace pictural.

\section{L'organisation picturale}

Pour plus de clarté, nous avons séparé ces terrasses en trois grandes parties - haute, moyenne et basse - (Figure 5) et défini chaque élément décoratif comme un motif. Nous avons ensuite regroupé les motifs en unités de lecture, désignées de A à J sur la Figure 5, chaque motif portant par ailleurs le numéro attribué par les archéologues de Copesco au moment de la restauration des terrasses (Echevarría López 2008). L'enregistrement fait alors n'est pas sans quelques imperfections (erreurs d'altitude notamment). Quant au lama numéroté 24 présent sur la terrasse 2, son numéro s'explique par la découverte tardive qui a été faite de lui. Pour donner un certain mouvement à l'ensemble du panneau, les artistes ont décalé chacun des motifs vers la droite, le long d'un axe oblique, orienté du nord au sud, lequel, sous l'effet de la perspective, donne l'impression d'être orienté du nord-est au sud-ouest. Cette impression est accentuée par l'emplacement des petites dalles insérées dans le mur de chaque terrasse, notamment vers 11 heures du matin, en raison de l'ombre produite par le soleil.

Les quatre terrasses supérieures du haut comportent huit lamas mâles et adultes $^{25}$, plus ou moins similaires, disposés les uns à côté des autres, sur quatre niveaux que nous avons considérés chacun comme une unité de lecture. L'un de ces lamas, le n' 24 , se situe sur un panneau disposé en saillie, qui le met particulièrement bien en valeur. Un autre lama, le $\mathrm{n}^{\circ} 1$, est placé juste au-dessus.

Viennent ensuite, sur les deux terrasses suivantes $\left(n^{\circ} 4,5\right)$, deux lamas adultes, vraisemblablement des femelles, car ils sont accompagnés de leurs petits (Paz Flores 2007 ; Echevarría López 2008, p. 39), soit quatre motifs plus ou moins similaires qui forment, là encore, une unité de lecture.

Les douze terrasses de la partie moyenne et basse numérotées de 7 à 18 mettent en scène comme on l'a vu deux caravanes de lamas adultes, vraisemblablement mâles, marchant plus ou moins côte à côte, sur différents niveaux, dont 
l'organisation générale reprend le même modèle que celui que nous venons de décrire avec, cette fois, un seul lama par terrasse. Les animaux y sont toutefois associés, sur une même file, par ensembles de trois, nombre que nous avons à nouveau considéré comme une unité de lecture. Ce regroupement est provoqué par un élément du relief qui vient interrompre l'apparente progression de la caravane. On trouve, par exemple, trois animaux dans la caravane du haut à droite (sur les terrasses $\mathrm{n}^{\circ} 7$ à 9), puis à nouveau trois animaux dans la file du bas (sur les terrasses $n^{\circ} 10$ à 12) limitée à droite par un affleurement rocheux (là encore de couleur blanche), placé exactement au centre du panneau mural ${ }^{26}$. La progression se poursuit ensuite vers le bas, avec trois autres animaux, là encore limités à droite, mais cette fois par les escaliers (terrasses $n^{\circ} 13$ à 15 ), et trois animaux qui viennent à nouveau buter sur une roche, localisée à droite et en contrebas (terrasses $\mathrm{n}^{\circ} 16$ à 18). Un petit personnage, qui pourrait être le berger conduisant la caravane de lamas ainsi constituée, a été figuré à l'extrémité de la terrasse inférieure $\mathrm{n}^{\circ} 19$ (Figure 7, $\mathrm{n}^{\circ}$ 4). Il forme, à lui seul, une nouvelle unité de lecture.

Ainsi, et en dépit de son apparente cohésion, l'ensemble des mosaïques de Choqek'iraw composerait des groupes picturaux distincts : des lamas adultes, vraisemblablement mâles, sur les quatre terrasses supérieures, des lamas femelles, accompagnées de leurs petits sur les deux autres terrasses juste en dessous et deux caravanes en mouvement, vraisemblablement composée de lamas mâles, la plupart jeunes, gravissant le versant de la montagne, depuis la rivière de l'Apurimac. Ces caravanes sont constituées de quatre petites troupes de lamas qui regroupent chacune trois animaux : la première en haut à droite (unité $\mathrm{F}$ ), la deuxième plus bas à gauche (unité $\mathrm{G}$ ), la troisième, au centre dans la continuité de la précédente (unité $\mathrm{H}$ ) et la quatrième tout en bas à droite (unité I).

Cette organisation des motifs le long du versant de la montagne ne semble pas fortuite et pourrait indiquer un code de lecture spécifique. En effet, à l'exception des terrasses de la partie haute $\left(\mathrm{n}^{\circ} 1\right.$ et 2$)$, qui ne comportent qu'un seul motif, on observe, de haut en bas, un ordre décroissant dans le nombre de motifs représentés sur chaque panneau, puisque de 3 sur les terrasses 3 et 4 , il passe à 2 pour les terrasses 5 et 6 et à 1 pour toutes les terrasses des parties moyenne et basse (de 7 à 19). Curieusement, le nombre de terrasses dont les motifs, selon notre analyse, forment unité, obéit à une organisation inverse : ainsi, alors que chacune des quatre premières unités du haut correspond à une seule terrasse, on observe, juste en dessous, une unité à deux terrasses (E), puis des unités à trois terrasses, tandis que la dernière (unité J) est constituée, à nouveau, par une seule terrasse $\left(\mathrm{n}^{\circ} 19\right)$.

Les chiffres 2, 3, 4 et 6 ont généralement dans les Andes une charge symbolique forte (Urton 2003). Les nombres pairs sont associés au dualisme andin, particulièrement évident dans les institutions et les cérémonies de la période inca (Bouysse-Cassagne 1978, 1987; Rostworowski 1983 ; Pärssinen 1992). Ainsi, dans nos terrasses, on retrouve une opposition entre les lamas vraisemblablement 
mâles des terrasses supérieures et les lamas femelles, placés juste en dessous. Le chiffre 3 est, lui, considéré comme " celui de la plénitude et le plus complet » (Urton 2003, p. 54). La division tripartite semble de fait avoir joué un rôle très important pour les Incas, notamment dans l'organisation spatiale des ceque de la vallée de Cuzco (Zuidema 1974-1976, 1978, 1995 ; Pärssinen 1992, pp. 155-169, 179-192). En effet, dans la capitale inca, la répartition des ceque au sein de chaque suyu s'effectuait en trois groupes de trois. Un seul suyu n'obéissait pas à cette règle puisque les quatorze ceque qu'il comprenait étaient assemblés en trois groupes divisés, respectivement en 4, 4 et 6 ceque ${ }^{27}$. Cette organisation tripartite semble particulièrement présente à Choqek'iraw où l'on retrouve le chiffre trois, à la fois dans le regroupement des lamas en unités des parties médiane et inférieure du complexe décoratif, mais aussi dans le nombre de terrasses du versant oriental qui sont également organisées en quatre unités de trois, soit douze. Trois est aussi le nombre de niches et fenêtres ornant la « maison des prêtres », et encore le nombre de pics couronnant le Cerro Wiracochan, vers lequel elle fait face, à $310^{\circ}$ au nord-ouest, et celui des trois sommets du Cerro San Cristobal qui lui est opposé. Cette répartition tripartite rappelle celle mentionnée par Zuidema (1992) pour les quatre saisons de trois mois chacune du calendrier agricole, où les lamas jouaient un rôle primordial.

Les terrasses étant, sur un autre plan, des lieux liés à l'irrigation et à la culture, on peut s'interroger sur la fonction de celles analysées ici. L'utilisation à des fins agricoles des terrasses décorées et de celles, particulièrement imposantes, du versant oriental, demeure problématique d'abord en ce qui concerne les produits qui auraient pu y être cultivés ${ }^{28}$. Peut-être s'agissait-il de maïs à caractère sacré, comme sur l'île du Soleil, au cœur du lac Titicaca, " où les grains qui y étaient obtenus étaient distribués à travers tout l'empire, comme manifestation physique de sa nature divine et comme un symbole, pour les peuples soumis que l'État inca contrôlait, de la production de cette plante sacrée »" ${ }^{29}$ (Bauer et Stanich 2003, p. 92) et associée au Soleil (Murra 1975a). Mais se pose alors le problème de la visibilité des motifs en mosaïques au moment de la maturation des plantes, les plants de maïs ayant pu atteindre près de $1,70 \mathrm{~m}$ de haut. Rien ne dit toutefois que les motifs devaient effectivement être vus tout au long de l'année. Peut-être ne l'étaient-ils qu'à des dates spécifiques - telles les solstices, équinoxes ou l'apparition des Pléiades - liées au calendrier agricole, car leur simple présence suffĩsait peut-être à conférer aux plantes auxquelles ils étaient associés un caractère sacré, similaire à celui du maïs de l'île du Soleil. Il est aussi envisageable, comme cela nous a été suggéré (Sophie Desrosiers communication personnelle), qu'à l'image des murs du Temple du Soleil, au Cuzco, nos lamas aient été recouverts de plaques d'or afin de capter les rayons du soleil couchant et d'en accentuer ainsi l'effet visuel ${ }^{30}$. Cela n'aurait d'ailleurs rien d'étonnant car, pour Bonavia (1974, p. 145), l'or comme les tissus servait à orner les façades de certains édifices, leur conférant en particulier une dimension religieuse. 
La couleur blanche des lamas représentés sur les mosaïques de Choqek'iraw est en tout cas révélatrice de leur force religieuse, car elle renvoie à la fois au soleil et au calendrier agricole, ce qui mérite quelques précisions. Au sujet de l'organisation des sacrifices dans la cité du Cuzco et en se fondant sur l'analyse des textes des chroniqueurs comme Cobo ou Guaman Poma, Zuidema (1989, pp. 424-429 ; 1992, pp. 63, 65) écrit :

La plupart des informations systématiques concernent le sacrifice des lamas, tel qu'il était organisé lors des rituels calendaires du Cuzco, la capitale de l'État inca. Les lamas étaient sélectionnés en fonction de leur race, leur couleur, leur sexe, leur âge, la hiérarchie ou l'objectif recherché, des distinctions liées à la fertilité, la stérilité, l'état sauvage ou domestique de chaque animal. [...] Le système de sacrifice le plus répandu au Cuzco impliquait la mise à mort de cent lamas au début de chaque mois, en sélectionnant des lamas de différentes couleurs pour chacune des quatre saisons. [...] Durant la saison des semailles, plus ou moins autour des mois de septembre, octobre et novembre, des lamas porteurs d'une longue toison blanche étaient choisis afin de renforcer la chaleur du soleil et sa capacité à envoyer la pluie. Durant la saison des moissons, c'est-à-dire à partir de l'équinoxe du mois de mars jusqu'à début juin, des lamas de différentes couleurs étaient sacrifiés en l'honneur du Tonnerre. Bien qu'aucune raison ne soit donnée quant à ce choix et que plus de deux couleurs fussent alors sélectionnées, des distinctions binaires de couleurs étaient associées au Tonnerre. Au cours des saisons intermédiaires, les dates des solstices incluses, lorsqu'on entreprenait peu ou pas d'activités agricoles, des guanacos (lamas sauvages) ou des lamas ressemblant à des guanacos, de couleur brune, étaient sacrifiés. Ils étaient consacrés au dieu Viracocha que les Espagnols appelaient le « Créateur ». [...] Les oppositions annuelles étaient donc les suivantes : la domesticité du Soleil et du Tonnerre s'opposait aux aspects sauvages de Viracocha et, à l'intérieur de cette opposition, l'affermissement du Soleil allait de pair avec un affaiblissement concomitant du Tonnerre au moment des semailles, et l'affaiblissement du Soleil avec le renforcement du Tonnerre durant le temps des moissons.

Ce même auteur (ibid., p. 65) ajoute plus loin que « des vieux lamas noirs jouaient un rôle important au cours de la période de quatre mois organisée symétriquement autour du solstice de décembre, au moment où les jeunes initiés de la noblesse de Cusco étaient mis en contact avec leurs ancêtres (Molina 1989 [1574], pp. 98-110) ». Zuidema souligne également qu'un mois avant cette période de quatre mois, " un lama noir était attaché à un poteau de la place du Cuzco afin de pleurer pour provoquer la pluie (Polo 1916 ; Guaman Poma 1987, pp. 256-257). Cette cérémonie se déroulait au moment où la Voie Lactée et la constellation du Lama (qu'elle est censée abriter), disparaissaient du ciel nocturne ». Il poursuit en indiquant que, six mois plus tard, " un rituel similaire se déroulait sur la place avec un lama blanc. Il ne pleurait pas, mais, avec ses pattes, il foulait un vase de bière de maïs, annonçant ainsi la saison sèche (Cobo p. 214) » (Zuidema 1992, p. 67).

Dans un autre article portant sur le même thème, Zuidema (2005, p. 93) précise son interprétation du texte original, relatif au lama blanc en écrivant 
« qu'on lui donnait de la bière à boire, pour le remercier d'avoir apporté le fruit des récoltes jusqu'à la ville ». Il rajoute que les sacrifices de lamas noirs et blancs se déroulaient respectivement quand le Lama céleste, une constellation située près de la Croix du Sud, disparaissait vers minuit (en octobre) et lorsqu'elle était visible durant toute la nuit (en avril), six mois plus tard. Dans son texte de 1992, Zuidema écrit «qu'un lama d'une espèce spécifique était alors choisi pour représenter le roi lui-même; il n'était jamais tué et était enterré avec pompe lorsqu'il mourrait pour des causes naturelles ». Dans l'article de 2005, ce même auteur précise que « durant ces deux mois de transition, les lamas et les produits agricoles sont associés ».

La narration des sacrifices de lamas de différentes couleurs à Cuzco, comme marqueurs de l'année agricole, est potentiellement riche de sens pour un site comme Choqek'iraw qui pourrait avoir connu des rituels proches de ceux de la capitale inca, mais dans un cadre plus régional, surtout s'il s'agit du domaine privé de l'Inca Tupac Yupanqui. Nous n'insisterons pas sur ces différentes fêtes qui mériteraient une analyse beaucoup plus approfondie, mais nous verrons plus loin que de nombreux autres indices semblent conférer aux mosaïques de Choqek'iraw un rôle dans le calendrier agricole, base de l'organisation des grandes fêtes annuelles dans toute cette région de l'empire.

\section{La division de l'espace pictural}

L'agencement des motifs obéit également à d'autres règles précises. Tous sont disposés le long de plusieurs lignes de fuite imaginaires qui, à une échelle locale, évoquent celles des ceque.

Au premier coup d'œil, tous les animaux semblent suivre une même direction vers le haut versant occidental de Choqek'iraw, selon un axe sud/nord, ou, sous l'effet de la perspective, sud-ouest/nord-est. Une observation plus détaillée permet cependant de déceler d'autres axes. Les plus apparents sont ceux formés par les animaux de la partie moyenne des terrasses (Figure 6a), ceux de la caravane du haut et les trois lamas de la partie haute de la caravane inférieure. Celui du haut (A-A') est orienté plus ou moins vers le nord, mais celui du bas (B-B') est légèrement décalé, de quelques degrés, vers le nord-est. Si l'on prolonge, par des lignes de fuite, la progression de ces deux troupes vers la gauche du panneau, les axes se rejoignent à un endroit précis du site (le point 0 sur notre croquis) qui vient juste d'être dégagé par les archéologues et se perd dans l'une des nombreuses terrasses du complexe. Peut-être ce point coïncide-t-il avec un affleurement rocheux à caractère sacré ou une source aujourd'hui tarie ou encore l'ancienne porte principale du site ? Quoi qu'il en soit, comme le suggèrent Valencia García (2004 ; Valencia García et al. 2005) et Paz Flores (2007), la caravane pourrait se diriger vers les villages des communautés agropastorales proches de Choqek'iraw, 
après avoir émergé symboliquement d'une source localisée, plus en contrebas, sur la partie droite des terrasses inférieures, à proximité d'un canal de drainage.

La progression des camélidés représentés sur les terrasses inférieures forme un second axe général (A-A' de la Figure 6b), beaucoup plus marqué, qui semble, cette fois, se diriger vers les escaliers des terrasses supérieures et, au-delà, vers les enclos situés à proximité du temple de l'hurin (voir aussi Axe A-A' de la Figure 3).

Pour revenir aux six camélidés de la partie moyenne dont il a déjà été question, ils sont disposés, de façon symétrique, de part et d'autre d'un axe central (D-D' de la Figure 6c) situé au milieu des terrasses, mais on peut les relier par paires autour d'axes perpendiculaires, dont chaque animal marque une extrémité. Cet ensemble qui se situe entre le haut et le bas pourrait marquer aussi une relation entre la gauche et la droite dans une conception quadripartite de l'espace. La notion de centre, taypi en aymara (Bertonio 1984 [1612], p. 340) ou chawpi en quechua (González Holguin 1989 [1608], p. 99), est évidemment un élément essentiel de l'organisation spatiale dualiste andine (Bouysse Cassagne 1978 ; Bouysse-Cassagne et Harris 1987, p. 20 ; Platt 1978) que l'on retrouve exprimée dans de nombreux domaines. Dans le cas des textiles, par exemple, c'est à la fois un lieu de rencontre ( $t^{\prime} i n k u$, en quechua) et de division des deux lés qui forment une tunique ou un poncho. Le centre joue un rôle double, ambivalent : il sépare, il crée deux parties et, en même temps, il les relie et constitue le «territoire » commun qu'elles partagent (Cereceda 1978, p. 1020 ; Zorn 1987). C'est aussi ce rôle de médiateur que semble jouer l'axe D-D' de la Figure 6c.

Il reste toutefois à déterminer quelle pouvait être la fonction de cette division de l'espace pictural en plusieurs unités, selon des axes de fuite spécifiques et apparemment liés à des accidents du relief, telles des roches ou des montagnes plus ou moins proches. Faut-il y déceler une organisation spatiale de tout le site, à l'image de celle reconstituée pour la vallée du Cuzco (Zuidema 1974-1976; 1978 ; 1995), Yucay (Molinié 1996) ou l'île de Taquile (Bellenger 2007)?

\section{L'inspiration textile dans les panneaux muraux de Choqek'iraw}

La disposition des motifs les uns par rapport aux autres laisse penser que la composition d'ensemble a peut-être été conçue selon des procédés utilisés pour confectionner une toile et, plus particulièrement, une tapisserie à chaîne verticale. Pour comprendre ce dont il s'agit, quelques rappels s'imposent. En règle générale, un tissu est fabriqué à partir de fils de chaîne et de fils de trame disposés perpendiculairement et entrelacés, plus ou moins savamment, selon différents procédés (Desrosiers 1992). Comme l'illustre D’Harcourt (2008 [1934], pp. 1920), « si les fils de trame et de chaîne sont semblables et si la tension et le serrage des duites les unes contre les autres correspondent à la tension et à l'écartement des fils de chaîne entre eux, le tissu formé sera uni et laissera apparaître en égale quantité sa chaîne et sa trame. Entre ces tissus à "armure unie", on distingue ceux 
à chaîne et trame continues et apparentes, ceux à chaîne et trame apparentes formées de fils discontinus et bouclés entre eux et les reps, c'est-à-dire des tissus où chaîne et trame sont continues, mais [où] un des deux éléments est caché, et la tapisserie est un des meilleurs exemples de ce procédé ». Toujours selon D'Harcourt (2008 [1934], p. 27), le reps désigne « un tissu à armature unie où la chaîne, élément passif, est entièrement recouverte par la trame [,] mais au lieu qu'une même duite parcourt la largeur entière de la pièce, elle est fragmentée et se compose de plusieurs fils successifs et de couleurs différentes, chacun ne croisant qu'un nombre restreint et variable de fils de chaîne avant de revenir sur lui-même. Autrement dit, la tapisserie se présente comme de petits tissages distincts et juxtaposés d'une même chaîne ").

Or c'est bien cette idée qu'évoquent les différents motifs figurant sur les terrasses de Choqek'iraw, où les pierres qui forment le parement du mur des terrasses sont placées verticalement, à l'image des fils de la chaîne d'un textile. C'est ce que nous essayons de suggérer en illustrant sur une même planche (Figure 8 ) les croquis du lama 18 de la terrasse 13 et du petit homme stylisé 25 de la terrasse 19 avec la photo détaillant une partie d'un sac à coca en laine, de culture inca, conservé au musée national d'archéologie et d'anthropologie de Lima. La partie inférieure illustre des lamas brodés au point de tige sur toile tissée, mais fait bien ressortir les fils de chaîne qui se prolongent ensuite par des pendeloques au fond du sac. Quoiqu'avec des techniques différentes, le concept serait le même. Nous verrons plus loin que divers autres sacs du même type, dont un repris d'après Lavallée et Lumbreras (1985, p. 34), présentent les mêmes caractéristiques.

Le panneau mural de Choqek'iraw évoque bien l'idée d'une grande étoffe en laine, étalée sur les hauts versants occidentaux du site. À l'image de certaines tuniques masculines ou $u m k u^{31}$ incas similaires à celle reproduite sur la Figure 9, les motifs sont disposés en étroites bandes parallèles et horizontales, chacune matérialisée par une terrasse et on pourrait imaginer la progression d'un tissage en suivant les terrasses de bas en haut dans un mouvement qui est aussi le sens d'ascension vers le sommet des caravanes représentées.

\section{Autres exemples de panneaux muraux inspirés de textiles}

« Tisser les murs » est un point de vue qui peut paraître étrange. Mais la transposition de décors textiles dans des panneaux muraux est un constat qui n'est pas nouveau dans les Andes. Comme l'écrit Lumbreras (1977, p. 16), « une grande partie du processus artistique ancien des Andes a un "caractère textile" ". Les premiers textiles décorés apparaissent au cours de la période archaïque, vers 2800 av. J.-C., et consistent aujourd'hui en de petits fragments découverts à Huaca Prieta, Ancon, Asia et sur plusieurs autres sites. Dès cette époque, les motifs sur d'autres supports que le textile sont de caractère textile, c'est-à-dire 


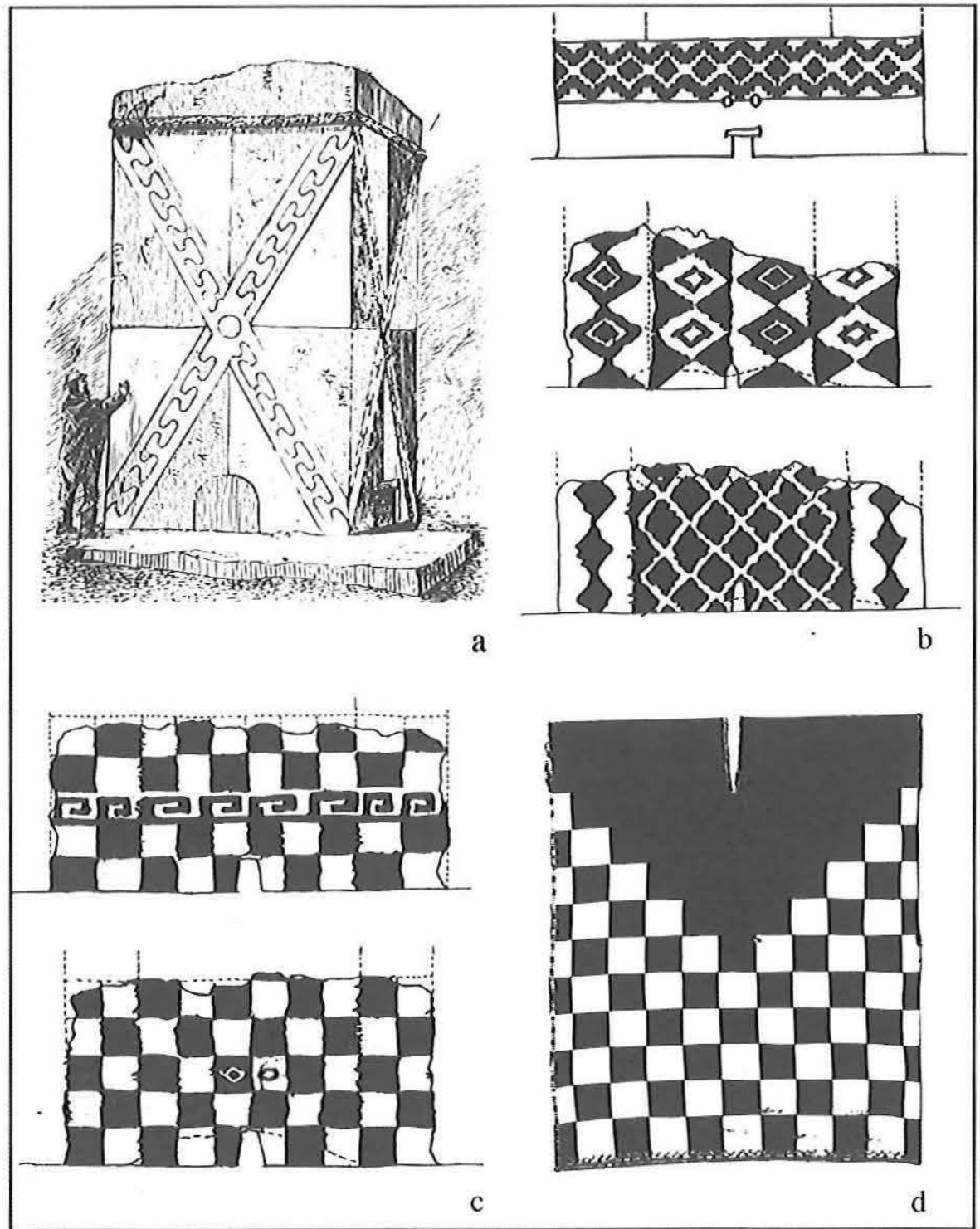

FIG. 9 - Décors de tombes d'autres régions andines dont pourraient s'inspirer les motifs retrouvés à Choqek'iraw.

a : chullpa ou " tour sépulcrale ", à la façade peinte de motifs de couleurs blanche et rouge, de la région de Palca en Bolivie (d'après Squier 1974 [1877], p. 133).

b et c: différents chullpa du site de Churi-Patilla (département d'Oruro, Bolivie), à la façade décorée de motifs géométriques peints en rouge et blanc (d'après Gisbert et al. 1996, pp. 49, 50).

d : tunique ou unku inca, à décor géométrique, hauteur : 0,95 m (Munich Staatliches Museum für Völkkunde, redessiné d'après Lavallée et Lumbreras 1985, p. 382). 
qu'ils paraissent obéir à une même structure que les tissus. Dans ces temps anciens, la technique dominante est celle de l'entrelacement des fils qui permet de former des motifs. Certains, purement géométriques, sont de petits losanges ou des lignes de chevrons et de carrés, d'autres, des représentations d'hommes, d'oiseaux, ou d'animaux.

Au sujet de l'art Chavin, qui se développe de 1300 à 500 avant notre ère, Lavallée et Lumbreras (1985, p. 26) observent également une certaine inspiration textile dans le traitement des motifs représentés sur la céramique ou sur les stèles. Leur analyse décrit des procédés décoratifs qui paraissent pertinents pour Choqek'iraw aussi : "Le tissu[,] par les caractéristiques mêmes de sa fabrication, favorise un traitement des images, bilatéral ou radial, à l'intérieur d'un dessin presque entièrement composé de lignes et d'angles qui ne font, en réalité, que suivre la direction des bases structurales du matériau, trame et chaîne ". À l'instar de Rowe $(1973$; 1978), ces auteurs remarquent que « les bases du style Chavin étaient déterminées par la symétrie, la répétition, le "module de largeur" et la réduction des motifs à une combinaison de lignes droites, de courbes simples et de volutes. La symétrie est essentiellement bilatérale, mais les deux moitiés présentent souvent des différences, l'important étant l'équilibre du dessin[.] Ce "module de largeur" est un élément textile structural où les motifs représentés se composent d'une série de bandes approximativement de même largeur, accolées et parallèles, où les traits naturels, non linéaires, des motifs représentés doivent s'adapter au cadre modulaire ». Les traits anatomiques des personnages figurés sont ainsi géométrisés. « L'une des constantes de ce modèle est la répétition de files de détails et de figures complètes, mais pas toujours exactement semblables, agencées selon l'espace disponible. Le procédé est donc le même que celui qui est employé pour le dessin d'un tissu fabriqué selon la technique de la tapisserie ou toute autre technique décorative structurale » (italiques de l'auteur PL). Ces mêmes auteurs soulignent encore que « l'art Chavín est donc essentiellement textile. La pierre et la céramique ne sont qu'un panneau (analogue à un tissu), sur lequel on grave le motif souhaité sans utiliser les possibilités plastiques qu'offrent ces matériaux. Les dieux et les démons du panthéon de Chavín n'ont donc pu avoir pour prototypes que des tissages, aussi pensons-nous qu'une des principales fonctions des centres cérémoniels devait être la production textile spécialisée, et les représentations de ce panthéon en pierre, sur divers autres supports (poterie, plaques d'or repoussées), ne sont que des adaptations de ces motifs textiles. [...] Les dieux de Chavín étaient représentés sur la pierre, mais ils ne sont pas en pierre : ils ne font que l'orner " (Lavallée et Lumbreras 1985, p. 31 et sq. ; italiques de l'auteur PL).

Mais qu'en était-il à Choqek'iraw, dont les principaux monuments ont été construits à l'époque inca, comme l'attestent leur style, la céramique trouvée et quelques sources ethnohistoriques récemment étudiées (Duffait 2007 ; Lecoq 2008)? Sur les terrasses décorées de ce site, chacun des motifs qui y ont été 
représentés est stylisé au maximum et s'inscrit dans un module de largeur comparable à celui décrit par Rowe (1973), cité par Lavallée et Lumbreras (1985), ce qui tendrait à conforter l'hypothèse d'une inspiration textile.

Le procédé est amplement connu au Pérou et tout au long de la période préhispanique après Chavín dont il a déjà été question. Ainsi, les sites Moche de la Huaca de la Luna ou del Brujo qui comptent quelques-uns des plus beaux panneaux muraux des Andes préhispaniques, semblent posséder une iconographie directement inspirée de motifs textiles (Bonavia 1974, p. 88 ; 1990a, p. 162 ; Franco Jordán et Vilela Puelles 2005; Uceda et al. 2006; Wright 2007). Les Chimus décoraient également les murs de leurs temples et palais de frises de tapisserie et, pour Sophie Desrosiers (communication personnelle), il est facile, en observant certains motifs ornant les murs de la citadelle Von Tschudi, de déterminer les techniques qui auraient été utilisées pour les tisser s'il s'était agi de véritables tissus.

Les sites de Pachacamac et de Huadca, au sud de Lima, obéissent aux mêmes règles, et la plupart des figures qui y sont représentées sont des copies évidentes de motifs textiles (Bonavia 1974, p. $124 ; 1990$ b, p. 166). Par la suite, «les Incas renforcèrent cette tendance en y intégrant leurs propres critères, encore une fois d'inspiration "textile" " (Lumbreras 1977, p. 22).

Les hautes terres des Andes centrales et méridionales semblent suivre une évolution comparable à celle de la côte, bien que moins connue. Ainsi, les mausolées funéraires ou chullpa de Macaya, sur les hauts plateaux boliviens de la région d'Oruro, sur les anciennes terres aymara des Caranga, ont leurs murs peints à l'effigie de textiles précolombiens. Décrits par Squier (1974, p. 131) en 1877, ils ont, depuis, été étudiés par Gisbert et al. (1994; 1996) et Gisbert (2000) qui ont répertorié plus de dix motifs différents sur ces monuments, dont certains sont reproduits sur la Figure 9. On y trouve des décorations en forme de damiers, peints en rouge et blanc, des cercles concentriques, des croix ou des losanges disposés verticalement le long des murs, mais aussi des frises de losanges à dents ou triangulaires continus, peints en rouge, blanc, vert et noir, ou des combinaisons de motifs en forme de ligne horizontale avec une grecque ou des yeux. Ces différents ornements présentent d'étonnantes similitudes avec ceux des tuniques incas ou unku illustrés sur les anciennes gravures ou celles conservées dans différents musées, similitudes que les auteurs expliquent par le fait que les Aymara de cette région ont été conquis par l'Inca Tupac Inca Yupanqui (celui-là même qui avait Choqek'iraw pour résidence) et que des gouverneurs incas se trouvaient sur leur territoire. Ils en concluent que les dessins représentés indifféremment sur l'architecture, les textiles et la céramique sont les signes distinctifs des seigneurs aymara qui y sont enterrés. Les chullpa décorés de Carangas portent donc sur leurs murs les différents motifs des tissus que revêtait chacun des chefs disparus et qui recouvraient ensuite les monuments juste après leur construction ${ }^{32}$. De nombreux textiles ethnographiques reprennent certains 
de ces motifs, tel le losange à dents ou les losanges concentriques (Gisbert et al. 1996, p. 50).

Comme l'ont montré Murra (1975b) et plusieurs autres chercheurs (Lumbreras 1977, p. 10 ; Cereceda 1978; Gisbert et al. 1987; Zorn 1987; Franquemont et al. 1992 ; Frame 1994 ; Renard 1994 ; Desrosiers 1997), ces analogies n'ont rien d'étonnant, car les textiles avaient, dans les Andes, un rôle particulier. Ils constituaient un langage spécifique à part entière. Ils étaient les véhicules de la pensée, image et lieu d'exercice des pouvoirs de la société. Ils servaient aussi de monnaie d'échange, de symbole de statut social, de dot ou de sceau d'armistice. Les plus belles pièces servaient à habiller les huaca ou étaient brûlées symboliquement en guise d'offrande sacrificielle. Aucun événement - qu'il soit politique ou militaire, social ou religieux - n'était complet sans que des tissus ne soient donnés ou reçus, brûlés ou sacrifiés. Concernant la fonction religieuse du tissu, à la période inca, Rembur (1987, p. 147), en s'appuyant sur les ouvrages de divers chroniqueurs, ajoute que «dans les nouveaux territoires annexés, les temples dédiés au culte solaire et les bâtiments réservés aux aclla et mamacona sont les premiers implantés. En effet, il faut de grands panneaux de tissus pour orner les temples, les vêtements pour les prêtres, enfin des tissus pour être brûlés en offrande, lors des sacrifices. Car le vêtement est considéré, dans la pensée inca, comme un double de l'être vivant : en agissant sur lui, on agit sur son propriétaire et, pour se libérer de la maladie, il suffit d'abandonner ses vêtements. En détruisant l'empire inca, les Espagnols en bouleversent les structures et mettent fin à cette production textile à la signification multiforme ${ }^{33}$. Sur le même thème, Itier (2008 p. 181) précise que :

Les vêtements n'étaient pas fabriqués à partir de pièces de tissu découpées et cousues, mais entièrement composées sur le métier à tisser, y compris les ornements et les granges. L'objectif du tisserand était de produire un objet achevé, car un habit ne pouvait être coupé. Les données ethnographiques modernes suggèrent en effet que les habits et même les sacs et les sacoches étaient considérés comme des êtres vivants, comme d'ailleurs la maison et d'autres entités que nous considèrerions comme inanimées. La nomenclature des parties du vêtement semble confirmer cette perception : une tunique, par exemple, comportait un " cœur", une "bouche ", des "viscères ", des " fesses », etc. Des ethnologues ont remarqué qu'on exprime parfois aujourd'hui l'idée selon laquelle un sac " est » un crapaud, une corde un serpent et une fronde un lézard.

Le fait de représenter un textile sur les hauts versants de Choqek'iraw, avec des motifs décoratifs empruntés au monde textile, paraît donc obéir à des considérations à la fois d'ordre symbolique et politique. Peut-être traduit-il le souhait, de la part des artistes qui en sont à l'origine ou du dirigeant qui a ordonné cette œuvre, d'assimiler Choqek'iraw à un huaca régional, en le revêtant d'un unku, représentant ainsi son caractère sacré ou l'émanation du huaca du même nom, localisé au nord-est de Cuzco, ainsi que l'ont mentionné Zuidema (1974-1976; 1978) et 
Bauer (2000). Une telle hypothèse pourrait expliquer la magnificence de ce site et des différents monuments qui y ont été érigés, avec pour but de proclamer la puissance symbolique du pouvoir à travers l'empire inca (Bray 2008). Si tel est le cas, il resterait à déterminer si ce site était effectivement lié à la production de textiles, comme le suggéraient Lavallée et Lumbreras (1985) à propos des principaux centres rituels andins. Selon nous, et comme nous l'avons déjà dit plus haut, la découverte, dans plusieurs kallanka du S.9, de nombreux fuseaux n'est, malgré tout, pas une preuve suffisante pour appuyer cette hypothèse.

\section{HYPOTHÈSES SUR LES REPRÉSENTATIONS PICTURALES DE CHOQEK'IRAW}

\section{Les motifs de camélidés}

Résidence royale, Choqek'iraw devait refléter le pouvoir du souverain aux yeux du peuple et des pèlerins qui se rendaient sur le site lors des cérémonies (solstices, apparition des Pléiades ou de la pleine lune, labourages, semailles et récoltes) qui devaient ponctuer les grands moments de l'année, comme c'est encore le cas aujourd'hui, dans d'autres parties des Andes (Karsten 1957, p. 206 ; Poole 1982 ; Bauer et Stanish 2003 ; Bray 2008). L'éclat et la complexité des différents édifices qui y furent construits (tels les bâtiments des secteurs centraux et les grandes terrasses des versants orientaux et occidentaux) jouaient le même rôle, comme symboles matériels de l'empire inca (Williams 2008). Les résidents ou visiteurs connaissaient probablement le symbolisme des motifs iconographiques qui leur étaient présentés - notamment sur les mosaïques des versants occidentaux - pour les avoir vus sur d'autres supports d'utilisation plus courante, comme la céramique, le bois, l'orfèvrerie ou l'art rupestre. Ils savaient également que les lamas avaient un rôle essentiel dans la plupart des fêtes qui accompagnaient la vie quotidienne des Incas.

Ne connaissant pas l'écriture, les artistes donnaient aux dessins et aux couleurs des valeurs symboliques (Bray 2008) dont le sens devait être conforme aux mythes fondateurs et aux récits des origines sacrées de l'Inca.

Les lamas sont ainsi des éléments récurrents de la céramique de style inca, où ils servent à décorer des assiettes ou des plats. Ils sont généralement peints en noir, sur une surface engobée en rouge carmin et finement polie, et sont disposés en file indienne, encadrés, en haut et en bas, par une ligne brisée de couleur blanche. Ces camélidés sont souvent représentés sur une seule file, parfois sur deux superposées. Ils se dirigent tous dans le même sens ou vont dans des sens opposés quand il y a plusieurs registres (Baca Cosio 1989. Figure 10f à h). Parfois, la ligne brisée inférieure est remplacée par deux lignes croisées qui pourraient illustrer le chemin emprunté par les animaux. 
Plusieurs coupes ou keru en bois, du début du Xvi ${ }^{\mathrm{e}}$ siècle, conservées au musée Inka du Cuzco, présentent exactement les mêmes motifs (Flores Ochoa 1998, p. 20). Flores Ochoa souligne qu'à cette époque, « la décoration la plus significative se fonde sur des motifs linéaires, travaillés selon la technique de l'incision, avec des figures géométriques, où prédominent des carrés, des rectangles, des losanges et zigzags » (ibid., p. 14) et que, " dans certains cas, apparaissent des figures de lamas. Sur l'un de ces vases, un peu plus tardif, la superficie extérieure a été divisée en trois bandes horizontales, séparées par des espaces étroits, avec des losanges concentriques et triangulaires, de formes très semblables à ce que l'on observe dans certains textiles modernes » (ibid., p. 197).

On retrouve exactement la même scène de lamas en file indienne, semblant gravir une pente, sur de nombreux autres objets, dont un sac à coca de tradition inca, découvert sur la côte sud du Pérou et reproduit dans Lavallée et Lumbreras (1985, p. 340). Cette pièce que nous avons déjà mentionnée, tissée et brodée en laine et coton, est aujourd'hui exposée au musée national d'archéologie, d'anthropologie et d'histoire de Lima.

Plusieurs autres pièces appartenant à diverses traditions culturelles présentent les mêmes décors. L'une des plus significatives est la chuspa, de tradition inca, conservée dans ce même musée et reproduite par Lumbreras (1977), où les lamas sont cette fois présentés sur cinq files superposées : trois en haut, regroupant six lamas placés en file indienne, et deux en bas, avec huit lamas de couleur jaune sur rouge, jaune sur vert et blanc sur rouge pour le lama central (Figure 8e). La partie supérieure du sac est décorée de motifs géométriques en forme d'étoiles et d'autres évoquant des tokapu incas, ces compositions à panneaux rectangulaires incluant des motifs gométriques (Frame 2007) ; la partie inférieure comporte des franges de couleur rouge, jaune, verte et blanche ${ }^{34}$.

Le musée d'Ica possède également un sac à coca inca, assez similaire (Figure 8f), qui est conçu sur le même modèle que celui précédemment cité, à la différence que la partie inférieure du sac met en scène des lamas rehaussés de jaune sur un fond rouge ou vert pale, disposés par trois, de part et d'autre d'un axe central délimité par des motifs géométriques difficiles à identifier. La partie supérieure semble représenter des cervidés, la tête de couleur jaune et les pattes de couleur verte, identifiables à leurs bois stylisés, se détachant sur un fond rouge. Ceux-ci sont associés à des motifs en croix, de même couleur, qui illustrent vraisemblablement la chacana ou Croix du Sud, un motif récurrent de l'iconographie andine ${ }^{35}$.

Il convient de noter que ce motif de camélidés est très similaire à celui, représenté sur un sac de coton de tradition nazca, de la collection Schmidt (Leicht 1960, figure 15) dont il est peut-être inspiré (Figure 10a), ce qui montre la persistance de certains thèmes figuratifs à travers les siècles dans les Andes.

Des petits lamas stylisés ornent également le matériel inca, des traditions pacaje et caranga, originaire du nord du Chili et du sud de la Bolivie (Lecoq et 


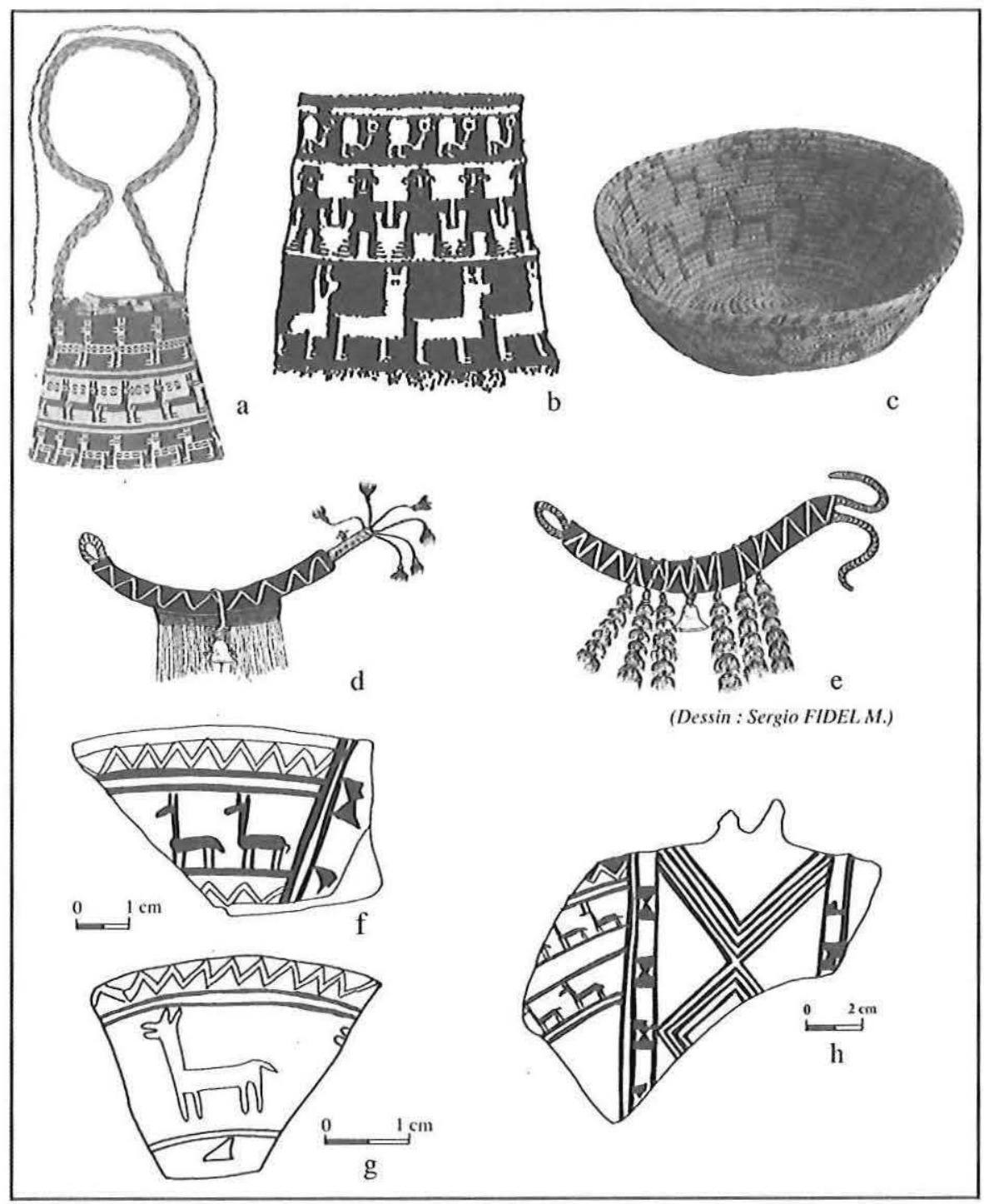

FIG. 10 - Figures de camélidés représentés sur divers supports et similaires à celles de Choqek'iraw. a : petit sac en laine et coton exécuté en tissage double, genre reps, décoré d'une suite de lamas, de couleur carmin (laine) et blanc (coton). Provenance : Nazca, collection Schmidt, Paris (D'Harcourt 2008, pl. XXX).

b : fragment de tissu double-étoffe, décoré de motifs de lamas (redessiné d'après Drake Moraga 2005, figure 63).

c : petit récipient en vannerie de la région d'Arica, Chili, décoré de lamas stylisés à deux têtes, période Intermédiaire récente (Museo universidad de Tarapacá-San Miguel de Azapa, Arica-Chile ; d'après Chacama 2008).

d et e : colliers à lamas, de type lincu-lincu (chemin-chemin) de la région de Ventilla (Potosi, Bolivie), tressés en laine de lama, de couleurs noire et blanche, avec des brins et des pompons de laine de mouton, de couleurs rouge et blanche (redessiné d'après Lecoq et Fidel 2003). f à $\mathrm{h}$ : céramiques de tradition inca décorées de motifs géométriques en forme de lignes brisées et de lamas stylisés (redessinées d'après Flores Ochoa et al. 1995, p. 197). 
Cespedes 1997 ; Gisbert et al. 1996). Le musée de l'université de Tarapacá-San Miguel de Azapa, à Arica au Chili, comprend, quant à lui, un bol en vannerie, de la période Intermédiaire récente et/ou inca (1000-1550), décoré de plusieurs lamas bicéphales stylisés (Figure 10c).

Pour conclure sur ce thème, on sait que des figurines de lamas stylisés, en or ou en argent, plus ou moins identiques à ceux figurés sur les terrasses de Choqek'iraw, étaient placées comme offrandes dans les tombes des personnages de haut rang, pour leur rendre hommage ou les accompagner dans l'au-delà. Cet animal jouissait et jouit encore d'un grand prestige auprès des populations andines auxquelles il fournissait une vaste gamme de produits, sans oublier son rôle de bête de bât (Murra 1975b). Sa fonction rituelle et psychopompe a par ailleurs été soulignée par de nombreux auteurs (Tschopik 1946 ; Pucher de Kroll 1950 ; Flores Ochoa 1974-1976, 1978 ; Flores Ochoa et al. 1995; Merlino et Rabey 1983 ; Tomoeda 1993, 1996 ; Bonavia 1996 ; Palacios Ríos 2000 ; Lecoq et Fidel 2000, 2003 ; Goepfert 2009, pp. 450-471). Pour sa part, Zuidema (1992) a montré le rôle fondamental qu'il occupait dans l'organisation du calendrier agraire inca.

Dans de nombreux cas, la représentation de camélidés qui est liée à la fertilité se rapporte à leur origine sacrée. Selon Flores Ochoa (1974-1976), les lamas et les alpacas sont sortis des profondeurs de la terre, en passant par des sources, des lagunes, des étangs, des fleuves et la mer elle-même, Mama Cocha. Ces courants et ces étendues d'eau sont des pacarina, des lieux d'origine (Duviols 1974-1976, pp. 283, 292). Les camélidés ont été prêtés à l'homme par le père créateur, Mañasqa Uywa, pour l'aider à subsister dans la puna et c'est la raison pour laquelle il faut les honorer en effectuant des cérémonies à certains moments de l'année. Or c'est justement d'une source que paraissent sortir les lamas composant «la » caravane de Choqek'iraw (Paz Flores 2007), encore que cela ne concerne, selon nous, que les lamas des terrasses inférieures. Les animaux des deux terrasses supérieures incarneraient plutôt, à nos yeux, les camélidés, à moitié sauvages, destinés à la fécondation des femelles et qui résident généralement dans les pâturages d'altitude, auprès des divinités agrestes tutélaires (voir aussi Flores Ochoa 1998) ${ }^{36}$. Ils seraient plus ou moins liés aux manifestations climatiques, notamment au tonnerre, autrefois incarné par Wiracocha, que semble évoquer le massif du même nom, vers lequel sont orientées les mosaïques aux lamas et les ouvertures de la "maison des prêtres ". La reproduction du cheptel serait figurée, symboliquement, par les deux lamas et leurs petits des terrasses 5 et 6 . C'est cette force fécondante ou enqa, que possèdent les lamas et les alpacas, qui explique qu'ils soient, très souvent, représentés dans une attitude cérémonielle et sacrée sous forme d'illa ${ }^{37}$ ou de conopa (Flores Ochoa 1974-1976). Il est donc possible que les lamas des mosaïques aient servi à glorifier la fertilité, en manifestant le pouvoir reproducteur des camélidés. 
Le rôle déterminant joué dans la religion andine par les camélidés n'est plus visible, pour les périodes anciennes, que dans l'iconographie de quelques objets et keru car, pour Flores Ochoa (1998, p. 196), leurs images auraient été, pour la plupart, détruites durant les campagnes d'extirpation de l'idolâtrie. Comme le souligne également Bonavia (1990b), les causes de la disparition de la plupart de ces œuvres d'art sont multiples, mais la folie iconoclaste des prêtres de la Colonie a sans doute été l'une des plus importantes. Durant le Xvi ${ }^{\mathrm{e}}$ siècle, sur les ordres des autorités espagnoles, les peintures et autres représentations d'anciennes divinités firent l'objet d'une destruction systématique. Cela transparaît clairement dans les instructions données par le vice-roi Toledo. Mais les mosaïques de Choqek'iraw ont survécu : elles sont un témoignage de la place déterminante des camélidés dans les pratiques et les croyances de la population locale et renvoient sans doute également au cycle des saisons, notamment à la saison des pluies, et au calendrier agricole, comme le propose Zuidema $(1989 ; 1992 ; 2005)$ à partir de l'étude des sacrifices pratiqués à Cuzco.

\section{Les motifs géométriques}

Les motifs en forme de lignes ondulées ou brisées sont plus diffíciles à interpréter, car ils sont très souvent représentés sur de nombreux supports, notamment textiles. Sur la céramique ou les coupes cérémonielles (keru), on les voit associés aux figures de camélidés et ils servent à décorer spécifiquement les rebords des vases (Figure 10f à h). Dans le registre du textile, les lignes ondulées peuvent border la frange inférieure de certaines tuniques masculines (uncu), comme celles à damiers, de tradition inca, conservées au musée de Staatliches für Völkerkunde de Munich (Figure 9d).

Aujourd'hui encore, les populations pastorales des régions de Macha et de Potosi, en Bolivie, ornent leurs frondes et les colliers rituels, dont ils parent les lamas de tête de chaque caravane, par des lignes brisées ou ondulées, dénommées link' $u$, « courbes » en quechua (Lara 2001, p. 144), et qui sont censées représenter les chemins zigzagants à travers la montagne (López et al. 1993, p. 240 ; Abercrombie 1998, p. 180). Détail plus révélateur encore, pour les bergers du Sud bolivien, chaque brin de laine symbolise l'un des chemins potentiels que peut emprunter la caravane (Lecoq et Fidel 2003). L'animal de tête porte donc sur lui une carte imagée de la région dont il doit parcourir les différents chemins (Figure 10d et e). Pour négocier son passage auprès des divinités agrestes tutélaires qui en contrôlent l'accès, cet animal leur " offire » quelques feuilles de coca conservées dans un petit sac ou chuspa accroché sur son poitrail (Lecoq et Fidel 2003, p. 46).

Le même motif peut faire référence aux méandres des fleuves et, par analogie, à l'eau, aux ondulations d'un serpent et à l'arc-en-ciel (Zuidema 1974-1976, p. 216). À ce sujet, Reinhard (1985), puis Williams, précisent que «l'eau, le 
liquide fondamental de l'activité agricole, est perçue comme coulant des lacs et des versants de la haute montagne pour éventuellement parvenir à la mer d'où il monte au ciel et de là, est à nouveau redistribué sous la forme de pluies estivales qui irriguent les montagnes sacrées, objet des vénérations des communautés » (Williams 2008, p. 59). Au sujet du serpent ou amaru, ce dernier auteur ajoute que « diverses sources soulignent son importance dans la cosmologie inca. La référence la plus notable est donnée par Guaman Poma (1936 [1615]), lorsqu'il signale que les Incas descendent des amaru, qui sont donc considérés comme les ancêtres des Incas » (ibid.). Enfin ce même auteur écrit également que "pour Meddens (2002) [et 2006, pp. 52-54], la transformation de l'amaru en pierre dans le récit de Huarochiri est importante, car elle exprime le lien entre le serpent, l'eau et la pierre, ainsi que l'apparition de l'amaru dans les moments de grandes transformations vers un nouveau monde. L'amaru est similaire à l'anaconda qui, dans son essence, est associé aux notions centrales de la cosmologie inca, comme les ancêtres, le ciel, les fleuves, les précipitations, les canaux d'irrigation, les pierres, les cavernes et la fertilité » (ibid.).

Toujours, sur ce même thème, Frame (1994, p. 321) souligne qu'« il y a une relation évidente entre les serpents et les pièces textiles. Les éléments individuels ressemblent aux serpents, car ils sont linéaires et flexibles. Et les serpents apparaissent souvent en ondulant ou en se mouvant en spirale, qui est le sens donné à plusieurs éléments des structures textiles ; ils muent également, en laissant derrière eux une couche transparente... Et il existe un parallèle entre une peau dont on peut se débarrasser et les tissus ou les toiles qui recouvrent le corps, et que l'on peut enlever ».

Les participants aux taqui dansaient également en zigzaguant. Rappelons que les taqui sont de grandes processions solennelles qui se déroulaient au rythme des flûtes et des tambours, au Cuzco, lors de cérémonies comme celle du solstice de décembre qui avait lieu en l'honneur des lacs d'où seraient sortis les camélidés (Duviols 1974-1976, p. 283 ; Zuidema 1974-1976 ; Zuidema et Urton 1976, p. 70 ; Tomoeda 1993, 1998). Aujourd'hui encore, les participants à la danse des wauqö ou pikkillu (les flûtes de pans), organisée dans le Nord Potosi en Bolivie, à la fin de la saison des pluies, suivent le mouvement ondulant d'un serpent, chacun prenant tour à tour la tête de la farandole. Ils miment aussi le comportement des lamas d'une caravane en route vers les vallées et, de cette façon, ils affirment l'unité de leur territoire communautaire (Zeguarra Choque et Puma Laime 2000). Stobart (1988 ; 1996a) ajoute que les jeunes danseurs, tout d'abord placés en fin de cortège - et plus symboliquement à la queue du serpent -, puis à sa tête, incarnent la génération montante qui sera progressivement amenée à remplacer les anciens dirigeants, un peu peut-être comme les jeunes lamas des deux caravanes de Choqek'iraw montent vers les pâturages d'altitude pour rivaliser avec leurs aînés.

Les damiers sont, en ce qui les concerne, des motifs récurrents de l'art andin que l'on trouve sur la plupart des supports. Ils ornent de nombreux aryballes 
incas et divers textiles. On en trouve par exemple sur un petit lama de laine, placé sur un coussin, découvert dans une tombe Chancay (Flores Ochoa et al. 1995, p. 188). Ils sont également représentés sur les murs d'anciens temples, comme ceux décrits par Bonavia (1990a et b) à Moche et Paramonga ou par Squier (1974 [1877]), Gisbert et al. (1994; 1996) et Gisbert (2000) à Macaya, en Bolivie. Concernant encore ces damiers, il apparaît que les textiles qui recouvrent les chullpa de Carangas reprennent les motifs reproduits sur les unku et d'autres pièces tissées, notamment ceux des communautés Q'ero du nord de Cuzco étudiées par Silverman-Proust (cité in Gisbert et al. 1996, p. 50), où l'on retrouve les mêmes couleurs : rouge, noir, vert foncé et blanc. Les motifs y sont faits à partir de losanges (qui sont une autre forme de damier) et de lignes diagonales émergentes et divergentes. "Le losange renverrait au Soleil sous des aspects divers, correspondant aux différents moments de sa course journalière, représentés par certains ornements : 1 Inti Llogsimushan ou Soleil du matin, 2 Hatun Inti, ou Soleil de midi, 3 Inti Chinkapushan ou Soleil du soir et 4 Inti Cocha [Soleillagune] ou Soleil de minuit. Celui-ci, dont le nom est composé de soleil-eau, est évidemment un pur concept » (ibid.) ${ }^{38}$. Est-ce à dire que les damiers reproduits à Choqek'iraw sont une évocation d'un aspect du Soleil ? C'est possible, compte tenu de leur localisation face à l'astre couchant et au caractère apparemment solaire de tout le site.

Par ailleurs, ces mêmes motifs en damiers évoquent les tocapu incas qui, lorsqu'ils étaient placés sur les vêtements des nobles, servaient à identifier leur rang et leur lignage (Gisbert et al. 1996, p. 49). Ils reflétaient de fait la structure de la société andine (Frame 2007) et, dans certains cas, « les provinces conquises ou gouvernées par celui qui portait le vêtement » (Itier 2008, p. 152). Il est également fait état d'une carte tissée sur un vêtement d'apparat (cumbi) que l'Inca Pachacuti aurait donné à l'ambassadeur Lukalarama, rappelant la représentation textile des divisions sociales, territoriales et cosmiques que l'on peut trouver sur les textiles modernes (Platt et al. 2006, p. 72-74). En raison de leur apparence ondulée et adaptée aux versants irréguliers de la montagne, les damiers représentés sur l'une des terrasses supérieures de Choqek'iraw semblent aussi faire allusion à un support textile flexible, qui prendrait vie sous l'effet du vent.

Il est difficile de donner une interprétation spécifique aux motifs représentés à Choqek'iraw sans disposer de données plus complètes sur ce site. Toutefois, le fait de retrouver associés, sur un même panneau, des damiers, des lignes brisées et des lamas stylisés, analogues à ceux reproduits sur les textiles, la céramique, les keru et d'autres supports, semble montrer qu'il s'agissait de thèmes familiers aux personnes qui vivaient ou visitaient le site, thèmes entre lesquels domine la notion de fertilité liée à la saison des pluies et à la reproduction des animaux. Cette notion est un élément fondamental de la plupart des sociétés andines et sudaméricaines (Descola 2005, chap. IX). 


\section{Récits mythiques et motifs décoratifs}

Pour de nombreux auteurs (Bonavia 1974; Gisbert 1980; Hocquenghem 1987 ; Bourget 1990 ; Urbano 1993 ; Rostworowski 1993 ; Bouysse-Cassagne 1997a ; Makowski 2000), les scènes représentées sur les façades de certains monuments préhispaniques ou coloniaux, sur la céramique ou les textiles des Andes centrales ou méridionales pourraient se référer à des événements historiques ou légendaires. Les décors de la Huaca Ciao del Brujo, à Moche, ont même été envisagés comme faisant office de calendrier (Franco Jordán et Vilela Puelles 2005). Il a été par ailleurs suggéré que certains des motifs ornant la céramique ou l'orfèvrerie des cultures nariño et carchi des Andes colombiennes et équatoriennes pouvaient évoquer la constellation d'Orion (Karadimas 1999). En était-il de même à Choqek'iraw? Si oui, à quel événement spécifique, à quel corps ou phénomène céleste les mosaïques du versant occidental pourraient-elles faire référence?

Pucher de Kroll (1950, pp. 205-213) avait avancé l'hypothèse que les motifs en forme d'étoiles, reproduits sur la céramique de style yura du sud de la Bolivie, puissent illustrer des étoiles et la constellation d'un grand Lama céleste, appelée Yacana, Catachilay, Urcuchillay, Choqechinchay (Pucher de Kroll 1950, pp. 19-27, 71-82, 191-202; Zuidema et Urton 1976, pp. 62-63) ${ }^{39}$, une constellation que les populations pastorales locales font résider au cour de la Voie Lactée, non loin de la Croix du Sud. Berenguer (1999) a repris la même idée, en montrant que les pétroglyphes de camélidés gravés sur les roches du désert d'Atacama et, notamment, un lama à deux têtes similaire à celui représenté sur certains objets en vannerie du musée de l'université de Tarapacá-San Miguel de Azapa, Arica (Figure 10c), pourraient évoquer cette constellation, particulièrement bien visible à cette latitude. Nous avons vu que cet animal céleste jouait un rôle important lors de certaines fêtes liées au calendrier agricole (Zuidema $1989 ; 1992 ; 2005$ ).

L'ancien mythe du Lama céleste, le Yacana, retranscrit dans un manuscrit anonyme quechua du XVII ${ }^{e}$ siècle (Taylor 1974-1976, p. $241 ; 1980$ ), nous livre de précieuses données sur ce sujet, même si elles se rapportent à une autre région des Andes que celle où se situe Choqek'iraw.

La constellation que l'on désigne sous le nom de Yacana est le camac des lamas [l'âme fécondante des lamas]. Elle fait son apparition en traçant son cours au milieu du ciel. Nous, les hommes, la voyons s'approcher toute noire. La Yacana se promène à l'intérieur d'un fleuve [la Voie Lactée]. Elle est vraiment très grande, avec des yeux et un très long cou ; elle noircit de plus en plus en s'approchant. Voilà ce que les hommes appellent la Yacana.

La Yacana descendait boire de l'eau des sources; si un homme avait de la chance, elle tombait sur lui. Toute couverte de laine, elle l'écrasait ; alors, d'autres hommes arrachaient cette laine. Ce phénomène avait lieu la nuit. Le lendemain, à l'aube, ils apercevaient la laine qui avait été arrachée, bleue, blanche, noire et châtain ; il y avait un 
mélange de toutes les sortes. Si l'homme chanceux ne possédait pas de lamas, aussitôt il s'en achetait. Il adorait la laine de la Yacana à l'endroit où on l'avait vue, où on l'avait arrachée. Ensuite, il s'achetait un lama mâle et un lama femelle. Rien que ces deux lamas lui fournissaient deux ou trois milliers de lamas. Cela serait arrivé, autrefois, à beaucoup de gens dans toute cette province.

On dit qu'à minuit, sans que personne ne le sache, la Yacana boit toute l'eau de la mer. Si elle ne faisait pas cela, la mer nous engloutirait aussitôt tous.

La Yacana est précédée par une petite tache noire. On l'appelle Yutu ( La Perdrix »). La Yacana a également un enfant. Quand l'enfant tète, elle se réveille. Ensuite, il y a trois constellations qui se suivent en ligne droite ; on les appelle le Condor, le Suyuntuy ("Le Gallinacée ») et le Huaman ("Le Faucon »). Quand [les étoiles] que nous connaissons sous les noms de Cabrillas ("Les Pléiades») apparaissent toutes grandes, on croit que l'année sera très fertile et si, au contraire, elles apparaissent toutes petites, on croit qu'il y aura une grande pénurie. [...] Autrefois, seule une partie des gens adorait ces étoiles en tant que (puissances) qui animaient, qui formaient. Les autres rendaient un culte à ces huaca, lorsqu'elles se levaient, en croyant qu'ils allaient, de cette manière, accroître leurs forces et, à cette occasion, ils veillaient toute la nuit. ${ }^{40}$

Le sens de ce mythe et son rapport avec la cosmologie andine, l'eau, les sources et la fertilité des hommes et des animaux, la richesse et le symbolisme de la laine - et donc des textiles - ont fait l'objet de nombreuses analyses et commentaires sur lesquels nous ne reviendrons pas (Pucher de Krol 1950 ; Zuidema et Urton 1976 ; Zuidema 1992). On sait aujourd'hui que chacun des animaux qui y sont décrits est formé par des taches sombres de la Voie Lactée, constituées par des nuages de poussières interstellaires et que ce zodiaque en négatif sert à déterminer les dates-clés de la vie paysanne, comme les semailles, les récoltes (Zuidema et Urton 1976 ; Zuidema 1978, 1992, 2005 ; Bock et Zuidema 1990 ; Loza-Balsa 1995 ; Bauer et Dearborn 1998; Urton 2006), ou encore à guider bergers et caravaniers lors de leurs périples à travers la montagne.

On peut se demander dans quelle mesure les mosaïques de lamas de Choqek'iraw ne font pas référence à cette divinité céleste, liée aux montagnes enneigées, à la fertilité, aux sources et aux fleuves, comme l'envisageait Berenguer (1999) pour les pétroglyphes du désert d'Atacama ou Pucher de Kroll (1950) pour les pasteurs des régions méridionales de Bolivie? En effet, Choqek'iraw convient particulièrement bien à l'observation de ces corps célestes, notamment depuis la colline tronquée (l'ushmu) qui domine les secteurs méridionaux et la vallée de l'Apurimac. Lorsque la nuit tombe sur le site, les étoiles composant la constellation de la Croix du Sud sont les premières à scintiller dans le ciel nocturne des mois de juin à septembre. Elles sont suivies, quelques minutes plus tard - à 10-12 plus au nord -, par deux étoiles particulièrement brillantes, identifiées comme Alpha et Beta du Centaure dans le zodiaque européen et comme les yeux du Lama céleste, les Llamas Nawi, dans les Andes (Pucher de Kroll 1950, pp. 198201 ; Zuidema et Urton 1976 ; Urton 2006, p. 202). Si l'on se place, la nuit, face aux mosaïques, la caravane qui s'y trouve représentée semble, vue de loin et sous 
l'effet de la perspective, gravir le versant de la montagne proche de l'ushmu, dans un cheminement qui va de droite à gauche et du sud-ouest au nord-est, selon un axe comparable à celui de la Voie Lactée au moment du solstice septentrional de juin (Urton 2006). À cette époque, la Voie Lactée se situe plus ou moins à l'aplomb du site. Les yeux du Lama céleste semblent dominer l'ushmu et, au mois d'août, sa tête se prolonge au-dessus du Cerro Ampay et son corps, sur la montagne Corihuayrachina unissant ainsi symboliquement deux des massifs les plus importants de la région (Figure 6a).

À partir du solstice de décembre, en revanche, la Voie Lactée devrait s'étirer dans le ciel, du nord-ouest au sud-est, selon un axe opposé à celui de la rivière Apurimac qui coule au sud de Choqek'iraw, lui faisant ainsi écho grâce au même modèle d'organisation spatiale symbolique que celui décrit par Urton (2006, pp. 58-59) ou Reinhard (2002) pour la vallée du Cuzco, dont l'axe principal est le fleuve Vilcanota. À cette époque de l'année, la Yacana disparaît du ciel nocturne, pour ne réapparaître que six mois plus tard, déterminant ainsi le rythme des saisons (Zuidema et Urton 1976).

\section{CONSIDÉRATIONS FINALES}

L'hypothèse selon laquelle la Voie Lactée ait pu inspirer les artistes qui ont conçu les mosaïques de Choqek'iraw n'est pas totalement dénuée de fondement, car on sait que les anciennes populations andines observaient avec soin les astres et autres phénomènes célestes afin de déterminer le calendrier des activités agricoles. Aujourd'hui encore, selon Reinhard (2002, p. 32) et Carrera Balgas (communication personnelle, août 2009), les habitants de la région proche du Salkantay et des environs d'Abancay, non loin de Choqek'iraw, considèrent la figure du Lama céleste comme une divinité protectrice, liée à la reproduction des camélidés. Il apparaît donc qu'en dépit de cinq siècles de présence européenne, la façon de voir et de penser l'univers est demeurée à certains égards plus ou moins la même qu'à l'époque précolombienne.

On propose donc ici que les mosaïques de Choqek'iraw soient une évocation du grand Lama céleste, protecteur de tous les camélidés, et que le site de Choqek'iraw lui ait été, au moins en partie, consacré, comme la manifestation d'une force fécondante et androgyne, selon Zuidema (1992), liée à la procréation et à la multiplication des camélidés (Urton 2006, p. 201) et similaire à celle du soleil. D'ailleurs, comme le précise Taylor (1980, p. 253), on sait aujourd'hui, grâce aux commentaires du chroniqueur Cobo, que « le culte des étoiles prototypes des espèces était pratiqué surtout par ceux qui avaient besoin de protection, par exemple les propriétaires de lamas qui demandaient, sans doute, à la Yacana de protéger et d'augmenter leurs troupeaux. Les autres se rendaient à leurs fêtes, comme à celles des autres huaca, pour acquérir du mérite supplémentaire ». Nous 
avons vu, plus haut, l'importance que les Incas attachaient aux camélidés, notamment à ceux de couleur blanche, noire et brune. Ils étaient intimement associés à l'apparition du Lama céleste dans le ciel nocturne des mois d'avril à septembre (Zuidema $1989 ; 1992 ; 2005$ ).

Il paraît donc évident, compte tenu des indices recueillis, que ces mosaïques n'avaient pas qu'une fonction purement décorative, mais plutôt votive et rituelle, comparable à celle des grands panneaux muraux de certains temples côtiers ou à celles des chullpa de Macaya qui incarnaient le défunt qu'ils renfermaient. Comme nous l'avons déjà suggéré, ces mosaïques servaient probablement à diffuser le message du souverain auprès de la population locale réunie lors des grandes cérémonies qui devaient se dérouler à cet endroit et qui étaient d'autant plus imposantes que le site était une résidence royale, destinée à perpétuer la mémoire de l'Inca auprès des membres de sa panaca et du reste de la société. Cette hypothèse pourrait amplement justifier le choix des motifs qui sont figurés. Comme nous l'avons vu, qu'il s'agisse des lamas ou des motifs géométriques, tous sont emblématiques de la culture inca et des sociétés qui l'ont précédée et étaient donc connus des habitants.

\section{Les mosäques comme représentation codifiée de l'espace régional}

L'inspiration textile des motifs représentés sur les terrasses de Choqek'iraw a été signalée. La façon dont ils sont agencés dans l'espace pictural et sur la pente paraît obéir à un code spécifique inspiré peut-être du textile, mais ayant une " signification multiforme ", selon Rembur (1987) ou Itier (2008, pp. 151-152, 181). Nous savons que, dans les Andes, les textiles constituent un langage codifié et servaient aussi autrefois de symboles d'identité. Certains d'entre eux comme les talegas de la région d'Isluga au Chili (Cereceda 1978), les sacs de bergers ou costales (Cereceda 1987; Gisbert et al. 1987) ou les señal q'epi et 'unkhuña ou inkuña (Zorn 1987; Horta et Agüero 2009) ont été interprétés comme des représentations imagées des espaces régionaux environnants.

Comme le précise Bellenger (2007, pp. 265,266) au sujet des communautés de l'île de Taquile :

La représentation de l'espace peut prendre diverses formes et être transposée à divers supports : transposition d'un espace géographique sur une table d'offrande rituelle au moyen de la disposition des $k^{\prime} i n t u^{41}$, axes rituels qui génèrent le symbole des territoires sur les textiles. (Italiques de l'auteur PL)

Ces formes renvoient à une vaste gamme de notions cosmologiques et idéologiques, comme la place d'un village (llacta) ou d'un centre communautaire ou cérémoniel perçu comme un espace de rencontre et de médiation (Staller 2008).

À cet égard, quelques exemples ethnographiques, pris dans d'autres régions que celle de Choqek'iraw, confirment bien que, dans certaines communautés 
pastorales, les textiles, notamment les señal q'epi et 'umkhuña, servent à délimiter le territoire de chaque ayllu. On peut se demander s'il n'en était pas de même à Choqek'iraw. Selon Zorn (1987, pp. 494-495), dans la région de Macusani, du département de Puno, au Pérou, le señal q'epi est une grande pièce textile à caractère cérémoniel, utilisée par les pasteurs d'altitude lors des rituels de marquage des camélidés ou señalkuy. Un señal q'epi se présente généralement comme un gros ballot ${ }^{42}$, fait d'une grande pièce d'étoffe, contenant divers objets rituels utilisés pour la préparation des tables d'offrandes et de nombreux textiles, de taille plus petite, appelés 'unkhuña, généralement décorés de franges horizontales parallèles et d'autres motifs qui renvoient à certains éléments de l'espace régional, comme les plaines et les montagnes qui le composent. Ces pièces textiles servent à « envelopper, garder ou transporter des petits objets, des aliments ou de la coca, mais aussi des autels (mesas) » (ibid.) pour déposer les offrandes lors des rituels pastoraux. Les femmes de Macusani les distinguent par leur taille, leur couleur, l'organisation spatiale régionale à laquelle ils font référence et leur fonction.

Sur ce même thème, Bellenger (2007, p. 285) pose comme hypothèse, en s'appuyant sur un article d'Arnold et Yapita (1992, p. 55), que, lors de certains rituels, « les quatre angles d'une 'unkhuña cérémonielle sont le plus souvent décorés de pompons, que l'on appelle ses "fleurs". On les désigne parfois comme ses "étoiles", comme si l'on se référait à la grande pièce de textile placée sous les cieux, en haut [la voûte céleste]. On les nomme également "fenêtres", parce qu'ils constituent une ouverture entre deux mondes ». Pour Zorn (1987, pp. 519-520), à Macusani, ces «tissus ne représentent pas seulement la société andine et ses membres à divers niveaux, mais ils sont eux-mêmes l'un de ces "êtres" de la cosmogonie andine : ils partagent l'univers avec les êtres humains, les animaux, la Terre Mère et les esprits des montagnes et vivent dans le monde miniature du fardeau rituel des pasteurs ». Ces mêmes tissus participent au caractère sacré de la cérémonie de marquage des camélidés (Merlino et Rabey 1993, p. 153).

En guise d'hypothèse, ne pourrions-nous donc pas percevoir les mosaïques de Choqek'iraw comme un textile qui, mis à plat et posé sur le sol, pourrait être une représentation imagée de l'espace régional, un peu similaire à celle d'une 'unkhuña, où chaque lama serait assimilé à l'un de ces objets sacrés et codifiés (dont des lamas en graisse) qui y sont généralement placés lors de certains rituels agropastoraux liés à la reproduction et à la fertilité des camélidés ? Cette suggestion rappelle également celle proposée par Molinié (1996) pour les terrasses de Yucay qui seraient une représentation codifiée et symbolique de l'espace dualiste régional. Soulignons toutefois qu'il ne s'agit, là encore, que d'une piste de recherche qui reste à confirmer ${ }^{43}$. L'hypothèse est d'autant plus séduisante que l'ushmu de Choqek'iraw occupe le centre de plusieurs axes symboliques (ceque) qui se dirigent vers les principaux massifs environnants au sommet desquels Thibault Saintenoy (communication personnelle, septembre 2009) a identifié des vestiges archéologiques, certains à caractère rituel. 


\section{Les mosaïques comme espace de médiation}

Comme entrevu plus haut, un textile peut matérialiser la place d'un village, lieu de concordance entre différents espaces. Dans de nombreuses parties des Andes, la place est le centre privilégié des différentes manifestations communautaires au cours desquelles les responsables occupent des espaces spécifiques qui leur sont réservés en fonction de la situation géographique de leur ayllu d'appartenance. À Taquile, par exemple, Bellenger (2007, p. 284) indique que, lors des fêtes, les représentants de chaque secteur se déplacent [en file] avec leurs responsables et leurs groupes de musiciens. Il souligne que les participants pénètrent systématiquement sur la place depuis l'angle nord-est. « Après en avoir fait le tour, toujours dans le sens inverse des aiguilles d'une montre, ils commencent à effectuer un premier arrêt dans l'angle sud-est [contrairement à ce qu'écrit cet auteur, il s'agit plutôt de l'angle nord-ouest], et cela successivement dans chaque angle de la place, avant de se diriger vers le centre » (ibid., p. 109), allant donc du nord-est vers le centre.

Il se trouve que c'est ce même axe que suivent les lamas reproduits à Choqek'iraw, mais dans un mouvement inverse, qui va du bas vers le haut et du sud-ouest au nord-est, comme s'ils se déplaçaient du centre de la place du village, matérialisé par les terrasses médianes, pour aller vers l'angle nord-est, qui est symboliquement dominé par le Cerro Corihuayrachina ou Yanacocha, derrière lequel se lève le soleil au solstice de juin. Selon Bellenger (2007, pp. 284-288), la place centrale du village est « le lieu où se déploient les bandes de musiciens au cours de cette phase [finale] du rituel qui "ouvre" l'espace et permet d'alimenter la roue du temps. C'est ce qui permettrait d'expliquer que cette régénération, au centre de la place, où se concentre l'énergie des participants, se manifeste par un air de musique qui les accompagne également jusqu'à la fin du cérémoniel pour commencer un nouveau cycle annuel ». C'est aussi à cette notion de centre cosmique et énergétique, lié au concept de camac, à laquelle se réfère Staller (2008, p. 306), lorsqu'il parle du centre rituel d'une place, d'un village (llacta) ou d'un ushmu.

Bellenger (2007, pp. 284-294) met en évidence une nouvelle concordance entre l'espace textile et musical, en précisant que les pinkillu (les flûtes) de l'île de Taquile ont six orifices et que chaque orifice est assimilé à l'un des six suyu ou territoires de l'île. Pour illustrer ses propos, il reproduit schématiquement l'une de ces flûtes dont les six orifices, superposés, font penser aux six lamas des terrasses inférieures de Choqek'iraw, placés le long d'un même axe. Bien que les supports soient différents, le concept paraît être le même. Ainsi, toujours selon Bellenger (2007, p. 294), au moment de la fabrication de l'instrument, « la perforation des orifices s'effectue progressivement, en commençant vers le bas de l'instrument, qui correspond symboliquement à l'espace des ancêtres, pour se poursuivre vers 
la partie supérieure de la flûte, assimilée à l'espace des vivants, comme si la progression des perforations retraçait un retour à la vie, depuis le monde du silence ». Pour cet auteur, il s'agirait « d'un cheminement initiatique qui conduit jusqu'à l'ouverture de la flûte par laquelle sera insufflée l'énergie vitale » (ibid.). L'exemple est d'autant plus intéressant que les instruments à vent sont, dans les Andes, associés à la période des pluies, aux morts et à l'inframonde (Harris 1983; Bouysse-Cassagne et Harris 1987 ; Stobart 1996b ; Parejo-Coudert 2001).

Nous avons vu que, si les lamas avaient été conçus selon un procédé textile, ils auraient été tissés du bas vers le haut, dans un mouvement en fait similaire à celui utilisé pour fabriquer une flûte, qui exprime aussi une progression linéaire. Et c'est également en jouant un air de flûte - dont le nom varie selon la région : quena, pinkillo ou rôlano (Parejo-Coudert 2001) - que le berger accompagne sa caravane lors des voyages de troc (Lecoq 1987 ; Stobart 1988).

Dans plusieurs articles (Lecoq 1987; Lecoq et Fidel 2003), nous avons montré comment le voyage de certaines caravanes était vu comme un cheminement initiatique et, selon Merlino et Rabey (1983, p. 163), comme le rite majeur des bergers. Il leur permet d'acquérir les produits indispensables à leur survie, tout en traversant des terrains inhospitaliers, peuplés de divinités plus ou moins hostiles, avec lesquelles ils doivent négocier leur passage. Pour le jeune apprenti qui les accompagne, c'est un rite de passage entre adolescence et âge adulte, grâce auquel il pourra constituer son réseau de partenaires de troc. Les lamas, et notamment les trois lamas de tête, sont les médiateurs entre le monde domestiqué des hommes et celui, sauvage, des divinités agrestes tutélaires. La caravane est ainsi perçue comme un trait d'union entre différentes régions, mais aussi différents espaces-temps, présents et passés.

C'est cette même idée de médiation entre des mondes opposés, mais complémentaires, du haut et du bas, de l'est et de l'ouest, que pourraient exprimer les mosaïques de Choqek'iraw. D'un point de vue géographique et topographique, elles se situent à l'aplomb du profond canyon de l'Apurimac et sont dominées par les hautes cimes enneigées des principaux apu régionaux, et entre l'ushmu (au sud) et la montagne de Choqek'iraw (au nord) (Figure 1). Elles marquent également l'union entre la rivière terrestre, Apurimac, et la rivière céleste représentée par la Voie Lactée. La présence d'une source et d'un canal, dans la partie basse des terrasses du secteur 8 , renforce cette idée. En effet, nous avons déjà dit que les sources étaient considérées comme des lieux d'origine des camélidés (pacarina) et les canaux comme une matérialisation des fleuves souterrains, mais aussi de l'inframonde, domaine des morts et des ancêtres.

Le cheminement du bas vers le haut confirme le rôle rituel joué par la colline tronquée, l'ushmu. La découverte, à son sommet, de petites structures en pierre, en forme de damiers, orientées vers certaines montagnes sacrées environnantes et, peut-être, aussi en fonction de l'apparition du soleil au moment des solstices et des équinoxes souligne également le rôle d'observatoire qui paraît avoir été attribué à 
ce monticule au sommet aplani. Cet ushmu constituerait ainsi un véritable axis mundi, unissant le bas, le haut et les différentes directions de l'univers (Zuidema 1989 ; Staller 2008, pp. 283, 306), ce qui confirme, d'une certaine façon, le rôle de médiation du panneau mural. L'orientation des motifs face au soleil couchant (des mois de juin à septembre et, peut-être, d'autres mois de l'année, ainsi que nous avons pu observer) et leur agencement selon certaines lignes de fuite vont également dans ce sens.

\section{Les mosaïques comme calendrier rituel}

On peut enfin se demander si, dans une certaine mesure, à l'image des ceque de Cuzco (Zuidema 1978; 1992 ; 1995 ; 2008a), les mosaïques de Choqek'iraw n'auraient pas pu jouer un rôle un peu comparable à celui d'un calendrier agropastoral, destiné à organiser l'activité des communautés régionales tout au long de l'année. Sur l'île de Taquile, les trois axes majeurs (est-ouest, sud-est/nordouest et nord-est/sud-ouest) qui symbolisent l'espace sacré et définissent les six territoires de l'île sont figurés sur tous les textiles (Bellenger 2007, p. 249). Les périodes de l'année sont représentées sur de grandes ceintures appelées calendriers, tissées par les femmes, où l'on trouve des symboles représentant les différents aspects de la vie agricole et du calendrier festif de la communauté (ibid., p. 114 ; INC 2006, pp. 44-58).

Cet exemple évoque celui du manto paracas de Bronklyn (D'Harcourt 2008 [1934], pl. LXXX-XCVI), censé illustrer un calendrier, ou les tuniques de tradition wari, conservées au Dumbarton Oaks Museum qui, selon Bock et Zuidema (1990 ; Zuidema 2005, pp. 95-96, 2008b), auraient eu une fonction plus ou moins similaire. Les motifs de ces tuniques sont aussi représentés sur les céramiques de Conchopata et, comme l'observe Zuidema (2005, p. 96), « nous avons vraisemblablement affaire, dans tous les cas, à quelque chose ressemblant aux systèmes de ceque, particulièrement importants pour comprendre les concepts wari sur l'organisation politique et calendaire ». Sur la base d'analogies iconographiques avec la culture inca, ce même auteur souligne que « le calendrier, tel qu'il est représenté sur ces "tuniques royales", s'organisait probablement autour de fêtes similaires à celle du Capac raymi qui marquait la fin du solstice de décembre. En conclusion, il semble que les rituels représentés par les pièces d'art que j'étudie se focalisaient autour du solstice de décembre. Des rituels similaires devaient occuper le cœur des sociétés tiwanaku et wari en général. Dans le futur, nous devrions attacher plus d'attention aux rituels et à leurs expressions artistiques dans les schémas des calendriers » (ibid.). Cet exemple rappelle celui de la carte tissée sur un vêtement d'apparat offert par l'Inca Pachakuti à l'ambassadeur Lukalarama (Platt et al. 2006, pp. 73-74) ou le témoignage de Polo de Ondegardo qui, dans la seconde moitié du Xvl ${ }^{\mathrm{e}}$ siècle, « dit avoir vu, à Pocona, des cartes peintes qui représentaient le système des ceque locaux » (ibid., p. 73). 
En nous appuyant sur les exemples proposés par Zuidema (2005; 2008b), ne pourrions-nous pas envisager, en guise d'hypothèse, que les douze lamas formant les deux caravanes représentées à Choqek'iraw aient joué un rôle analogue à ceux des motifs géométriques et zoomorphes représentés sur les tuniques wari, et que chacun d'entre eux ait été lié à un moment donné de l'année, notamment lorsque le soleil couchant vient les éclairer et les mettre en lumière ? C'est d'autant plus plausible que, comme pour les motifs ornant les ceintures de l'île de Taquile, chaque animal a ses propres caractéristiques et pourrait donc très bien symboliser un mois spécifique de l'année. Si tel est le cas, ne pourrions-nous pas concevoir, là encore à titre d'hypothèse, que chaque terrasse ait été associée à une communauté spécifique chargée de s'occuper de la gestion de ce site cérémoniel, selon un système de prestations de services rotatifs, de type ayni ou minka? Aujourd'hui encore, les communautés paysannes de la région voisine d'Abancay ou des hauts plateaux du Pérou ou de Bolivie utilisent fréquemment ce système d'entraide pour entretenir les ouvrages d'intérêt commun : canaux d'irrigation, terrasses et routes.

Le fait que Choqek'iraw porte le même nom qu'un huaca de la vallée de Cuzco, dans l'Antisuyu, placé sur le quatrième ceque, au nord-est de la cité, et directement lié au solstice de juin, est aussi à prendre en considération, bien que, comme nous l'avons évoqué, les deux sites soient apparemment différents (Bauer 2000, pp. 94-96; Saintenoy communication personnelle, septembre 2009). Ce huaca semble avoir fait partie intégrante du culte solaire, ce qui pourrait également être le cas de Choqek'iraw, même si les données ethnohistoriques dont nous disposons sur ce site sont, pour le moment, insuffisantes pour conforter cette hypothèse.

Il convient donc d'être prudent, car bien que les hypothèses avancées reposent sur un large faisceau d'indices, l'état actuel des connaissances sur le site, encore partiellement enfoui sous la végétation, ne permet pas de les étayer véritablement. Les mosaïques qui ont été restaurées ne donnent qu'une vision partielle de leur contenu original. Les motifs en chevrons et les damiers des terrasses supérieures sont, en effet, séparés des figures de lamas par plusieurs terrasses qui auraient pu abriter d'autres scènes, aujourd'hui disparues ou encore enfouies sous terre.

\section{Bilan général}

Pour conclure, un bref bilan des données archéologiques et des hypothèses que nous venons de formuler s'impose, notamment afin de voir celles qui pourraient être appuyées par de plus amples recherches.

Les différents indices que nous avons réunis montrent tout d'abord que Choqek'irav était un établissement complexe, à caractère certainement rituel, vraisemblablement construit pour magnifier la puissance du souverain et perpétuer sa mémoire auprès de sa panaca après sa mort. L'emplacement paraît avoir 
été choisi et planifié avec soin, en fonction notamment des éléments du relief, comme le fleuve Apurimac, les montagnes et les glaciers environnants, ce qui suggère une relation étroite avec l'eau et la fertilité, comme le pense Ann Kendal (communication personnelle, juillet 2009). C'est aussi ce que semble démontrer l'orientation de la plupart des édifices et des deux grands complexes de terrasses des versants orientaux et occidentaux vers les principaux sommets régionaux, ou la place déterminante des fontaines et du réseau de canaux servant à les alimenter.

Le site se conforme au dualisme andin où tout est agencé selon un jeu de miroirs et d'oppositions multiples, qui évoque la notion de yanantin (Platt 1978) ; il renvoie à d'autres sites incas, comme les terrasses de Yucay (Molinié 1996), apparemment conçus selon le même modèle. Opposition entre les montagnes qui dominent Choqek'iraw et la vallée de l'Apurimac, jeu d'ombres et de lumière le long des versants oriental et occidental qui sont, tour à tour, éclairés le matin et l'après-midi ; opposition entre la moitié du haut et celle du bas que réunit symboliquement le principal canal traversant le site de part en part. Par sa localisation, ce canal sépare les deux versants, mais il unit les deux moitiés et joue le rôle de médiateur, tout en renvoyant à la notion d'équilibre, tara en aymara (Stobart 1996b). D'autres oppositions existent dans le nom de certaines montagnes. Ainsi, le Cerro Coriuayrachina (mot quechua renvoyant au four pour fondre l'or) évoque ce métal doré et inoxydable, analogue au soleil dont l'Inca est l'incarnation sur terre, mais aussi l'inframonde et les mines dont il est issu (Berthelot 1978; Bouysse Cassagne 1997b, 2004, 2005; Absi 2003). À l'inverse, son autre toponyme, Yanacocha ou " Lagune noire ", semble plutôt faire référence à l'eau des glaciers et à l'obscurité de la nuit, tout en rappelant l'existence de la riche mine d'or de la région de Cajamarca, au nord du Pérou. Les aspects miniers du site sur lesquels nous ne nous sommes pas attardés mériteraient d'être approfondis pour mieux comprendre le rôle véritable de Choqek'iraw à la période inca.

La disposition particulière de l'éminence tronquée qui se trouve au cœur des quartiers urbains de Choqek'iraw semble, pour sa part, conférer à ce site le rôle d'observatoire astronomique que l'on peut mettre en relation avec l'organisation du calendrier agricole. Certains indices, comme les structures en damiers trouvées à son sommet et leur orientation vers les principaux massifs environnants, laissent penser que l'ushmu aurait pu être le centre d'un réseau régional de ceque comparable à celui de la région de Cuzco. C'est aussi ce que suggère la localisation des deux massifs à trois pics des cerros Wiracochan et San Cristobal, le long d'un axe d'orientation nord-ouest/sud-est, similaire à celui du cours du Río Apurimac. Cet axe évoque celui, symbolique, qui organisait le dualisme des hauts plateaux boliviens au cours des périodes de dominations aymara, puis incas (BouysseCassagne 1978 ; 1987), ou celui joué par le Río Vilcanota, dans la vallée de Cuzco (Reinhard 2002; Urton 2006). On trouve le même type d'alignements symboliques et d'oppositions dans la disposition des motifs de camélidés représentés sur 
les terrasses du versant occidental de Choqek'iraw. Ainsi, la caravane semble monter des profondeurs de la vallée du Río Apurimac, au sud-ouest, vers la cime du Cerro Corihuayrachina, au nord-est, alors que le panneau mural est orienté vers les trois sommets du Cerro Wiracochan situé au nord-ouest.

En plus des oppositions binaires, le chiffre trois paraît avoir joué un rôle important sur le site, car on le discerne à la fois dans le nombre de pics couronnant les deux massifs, mais aussi dans la quantité de terrasses du versant occidental. Celles-ci ont pu être regroupées en quatre unités de trois niveaux, soit douze terrasses. Douze est le nombre de lamas composant les deux caravanes des mosaïques qui, là encore, sont regroupés en quatre unités de trois. On serait tenté de rapprocher ce chiffre du nombre de mois composant une année, ce qui tendrait à nouveau à conférer à Choqek'iraw le rôle d'un grand calendrier lié au cycle des saisons. La présence, à l'aplomb du site, de la constellation du Lama ou Yacana n'est peut-être pas, non plus, étrangère à cette fonction de calendrier, ni aux motifs de camélidés représentés sur les mosaïques ou à leur nombre. En effet, l'apparition de la Voie Lactée dans le ciel régional et la couleur blanche utilisée pour représenter les lamas pourraient bien faire écho aux grandes fêtes agricoles de Cuzco, notamment le Capac Raimi de décembre et l'Inti Raimi de juin, analysées par Zuidema (1992). Si tel est le cas, ne pourrait-on pas considérer l'un des deux lamas blancs, placés en haut du panneau mural, comme la représentation imagée de l'Inca en route vers le soleil levant au moment du solstice de juin ?

Enfin, la disposition, à la verticale, des pierres des murs de contentions des terrasses sur le versant occidental est atypique et ne peut qu'évoquer la trame d'un textile, notamment celle d'un $u n k u$, tunique généralement réservée aux membres de la noblesse inca. Comme ces riches étoffes servaient aussi à revêtir les idoles, on peut se demander dans quelle mesure les artistes qui ont décoré les terrasses n'ont pas cherché à assimiler la montagne de Choqek'iraw à un grand huaca régional. L'hypothèse est d'autant plus séduisante que l'on sait aujourd'hui, grâce aux travaux de Zuidema (2005), que certaines tuniques de hauts personnages wari-tiwanaku auraient pu servir de calendriers et que cette coutume de représenter les grands moments de l'année agricole sur des textiles semble s'être perpétuée au sein de certaines communautés actuelles des Andes, comme celle de Taquile.

Ces analogies multiformes ont, bien sûr, pu être figurées sur d'autres supports (textiles, céramiques, lithiques) en renvoyant aux mêmes conceptions. Mais, avec ses mosaïques en particulier, le site de Choqek'iraw a été sans aucun doute un important huaca régional, où était peut-être célébré le cycle des saisons et où, de ce fait, les lamas ne pouvaient qu'occuper une position centrale. *

* Manuscrit reçu en décembre 2008, accepté pour publication en mars 2010. 


\section{NOTES}

Je tiens à remercier les nombreuses personnes qui ont contribué à l'élaboration de cet article : Percy Paz (Copesco) pour les informations relatives à l'histoire de la découverte du site de Choqek'iraw, les responsables de Copesco pour m'avoir permis d'utiliser les cartes ici présentées, Thibault Saintenoy pour la mise au propre des cartes de localisation du site et ses suggestions quant à l'emplacement des principaux massifs régionaux, Gilda Carrera, responsable de la Casa de la Cultura d'Abancay, pour les informations sur l'ethnographie régionale, Ann Kendal pour ses suggestions quant au possible rôle symbolique de l'eau à Choqek'iraw et Nicolas Goepfert pour ses idées sur la place des offrandes de lamas dans certaines tombes. Ma reconnaissance va également à Sophie Desrosiers pour ses précieux conseils et ses explications sur les aspects techniques des textiles, Tom Zuidema pour m'avoir communiqué ses articles sur le rôle des lamas dans le calendrier rituel des Incas et de Tiwanaku, ainsi qu'Éric Taladoire pour sa lecture attentive, sans oublier les deux lecteurs anonymes du Journal de la Société des Américanistes qui ont lu, commenté, critiqué ce texte, suscitant ainsi sa restructuration et l'approfondissement de plusieurs thèmes ou idées.

1. Ce travail repose essentiellement sur des relevés de terrain et des photographies réalisés entre 2004 et 2006 à Choqek'iraw, lors de la fouille de deux structures d'habitat domestique, destinée à déterminer la fonction et l'ancienneté du site. L'analyse des mosaïques qui venaient à peine d'y être découvertes ne faisait pas partie de nos objectifs originaux, mais l'observation de certains détails dans le choix et l'agencement des motifs nous a conduit à nous y intéresser. Les difficultés d'accès à ces terrasses, leur mauvais état de conservation au moment de nos deux premiers séjours et leur éloignement de notre campement ne nous ont pas permis de répertorier chacun des motifs, ni de voir la façon dont ils étaient disposés les uns par rapport aux autres. Nous n'avons pas pu non plus obtenir une copie des plans d'ensemble que les architectes de Copesco (Convention Pérou-Unesco, organisme chargé de la gestion et de la valorisation du parc archéologique) devaient établir. Il s'agissait, toutefois, de la première description analytique de ces mosaïques. En 2005, un rapport a été rédigé par les chercheurs de Copesco chargés de la restauration des terrasses aux lamas et déposé à l'Institut national de la culture (Valencia Zenobio et al. 2005). Depuis lors, un ouvrage consacré à l'étude archéologique de l'art figuratif de Choqek'iraw a été publié par Echevarria López (2008) qui a travaillé, lui aussi, à la restauration du site. Une récente visite de Choqek'iraw, en août 2009, aujourd'hui placé sous la direction de l'Institut national de la culture, nous a permis de compléter et de réactualiser nos données et de répertorier l'emplacement exact, sur chaque terrasse, des différents motifs représentés.

2. Ce terme semble en réalité venir à la fois de l'aymara et du quechua. Ainsi, selon le dictionnaire aymara de Bertonio (1984 [1612], p. 89), choque désigne "l'or, le métal le plus riche». Dans le dictionnaire quechua de González Holguín (1989 [1608], p. 117), le terme chhoqquechani corichami signifie « donner et estimer »; il est synonyme de ccorichani et ccori désigne l'or (ibid.,p. 69). Quant au terme qquirao, il se traduit par "le berceau des enfants» (ibid., p. 310). Toutefois, dans le même dictionnaire, nous trouvons également le terme chhoqqueylla qui signifie "éclair de la foudre ", ce qui correspond à la brillance du métal. Chhoqqueylla ou chuquilla est aussi l'un des nombreux noms donnés à la divinité cosmique inca personnifiant des phénomènes distincts comme l'éclair, le tonnerre et la foudre (Rostworowski 1983, pp. 39-42 ; Itier 2008, pp. 121-123), "que l'on priait sous les noms de choqueilla, catuilla et intiillapa " (Bouysse-Cassagne 1997b, p. 103), ce qui tend à donner une autre dimension sacrée au site. Dans plusieurs articles consacrés au monde minier et à son symbolisme, Bouysse-Cassagne (1997b ; 1998 ; 2005) évoque la sacralité des métaux, l'or et l'argent notamment, qui, pour les Incas, étaient liés aux origines, aux ancêtres et au guerrier céleste l'Éclair ou Illapa. Les indices que nous avons pu recueillir sur ce site suggèrent que, bien que Choqek'iraw n'ait apparemment renfermé aucune mine, il participe à cette même religiosité. Par ailleurs, comme l'a montré Molinié (1996) dans un autre contexte, les toponymes sont souvent organisés selon une structure logique qui renvoie à différents éléments du paysage, comme les montagnes, les champs ou les terres cultivées, et ont aussi un sens symbolique plus difficile à déchiffrer sans l'aide d'autres données ethnographiques ou ethnohistoriques. 
3. En raison de sa localisation à proximité de Choqek'iraw, ce sommet semble avoir joué un rôle important pour les habitants, rôle que paraît souligner le nom qui lui est généralement attribué. En effet, selon González Holguín (1989 [1608], pp. 69, 195), le terme quechua corihuayrachina désigne le four (huayarachina) servant à faire fondre l'or (ccori). Il renvoie donc à des notions de richesse et d'or tiré du sous-sol (Bouysse-Cassagne 1997b, p. 102), comme le nom Choqek'iraw. Quant au terme yanacocha, il signifie la lagune (ccocha) noire (yana) (González Holguín 1989 [1608], pp. 65 et 365, respectivement), ce qui introduit une idée d'opposition symbolique entre la brillance de l'or et du soleil, d'un côté, et la noirceur de l'eau et de la nuit (et de l'inframonde), de l'autre, opposition sur laquelle nous reviendrons. Yanacocha est également le nom donné à une grande mine d'or de la région de Cajamarca, au nord du Pérou (la plus grande d'Amérique du Sud), exploitée depuis l'époque inca, ce qui renvoie à nouveau aux notions d'or et de monde minier auxquelles semble se rattacher tout le site. D'autres toponymes régionaux font également allusion à l'or et aux mines d'or et ont une connotation sacrée qui mériterait d'être approfondie. Par exemple, la cordillère de Vilcabamba se réfère au soleil puisque, selon le dictionnaire aymara de Bertonio (1984 [1612], tome II, p. 386), villca signifie " le Soleil, comme on l'appelait autrefois, et aujourd'hui on dit inti ». Villcamuta est aussi « un lieu de culte dédié au soleil et à d'autres idoles ", et villa cuti « le solstice de décembre, lorsque le soleil commence le trajet depuis le tropique du Capricorne jusqu'à celui du Cancer ». Villca est " également une chose médicinale, une chose que l'on prescrit comme purge, pour dormir... ». Pour de plus amples données sur les aspects rituels de la région de Vilcabamba et ceux liés aux mines et leur importance pour les Incas, le lecteur se reportera à Duffait (2007), Zuidema (2005, p. 92) et Bouysse-Cassagne (1997b ; 1998 ; 2005). En ce qui concerne la dénomination marcani, ce terme semble plutôt se référer, selon González Holguin (1989 [1612], p. 231), à marca " défenseur » ou " avocat protecteur » - González. Holguin mentionne également " la poutre maîtresse, au sommet de la maison "-, marcani signifiant " prendre dans ses bras, embrasser ", ce qui renverrait aux notions de respect et de protection attachées à la montagne. Selon le dictionnaire quechua-espagnol de l'Academia nacional de la lengua quechua (1995, p. 305), le terme marka signifie également « ville " ou " village ".

4. Comme le fait remarquer Duffait (2007, p. 168, note 70), au sujet de Machu Picchu, ces dénominations récentes sont arbitraires et sans fondement scientifique. Nous ne les utilisons ici que pour pouvoir mieux situer les principaux bâtiments du site, sans perdre de vue que ces termes peuvent dénaturer leur véritable fonction, que seule une fouille permettrait peut-être de préciser.

5. Pour les Incas comme pour les peuples qui les ont précédés, certains éléments du relief comme les pierres dressées, luanca (ou wank'a), ainsi que les lagunes, les arbres, avaient un caractère sacré et étaient vénérés car tous étaient considérés comme des huaca ou wak'a, " demeures des esprits " (Cobo 1964 [1653], p. 166 ; Garcilaso 1982 [1609], livre II, chap. IV ; Karsten 1957, pp. 180-183). D'après González Holguín (1989 [1608], p. 165), le terme huacca désigne des « idoles, figurines d'hommes et d'animaux que l'on porte avec soi » et huaccas muchhana « le lieu des idoles, un lieu de culte ». Itier (2008, pp. 121, 192) précise toutefois que le terme « wak'a se réfère à un homme des temps primordiaux s’étant transformé en un élément "naturel", souvent une montagne, à la faveur d'un événement extraordinaire dont il fut le protagoniste. Dans le présent, le wak'a personnifie l'espace sauvage entourant le village, à la fois dangereux et utile, puisque c'est dans ses sources et ses lacs que prennent naissance les canaux d'irrigation. Il est également matérialisé par une pierre transportable à laquelle on rend un culte [...] le wank'a, rocher produit de la lithomorphose d'un ancêtre fondateur d'ayllu ". Cet auteur indique également que «la foule des ancêtres et des wak' $a$ adorés dans l'empire s'intégrait en deux grandes structures inclusives, les wak'a étant censés descendre, les uns de Wiraqucha, les autres de l'Éclair. Le panthéon andin était donc dominé par une dyade qui structurait le monde en deux sphères opposées et complémentaires. Alors que les wak'a étaient des divinités locales, les "esprits" des lieux, Wiraqucha et l'Éclair étaient des divinités cosmiques ". Certains rochers, assimilés à des wank'a, reproduisaient la forme des massifs environnants, témoignant du culte que les Incas rendaient aux montagnes (Rostworowski 1983, p. 62 ; Van de Guchte 1990 ; Meddens 2006). Ils pouvaient également servir d'observatoires (Zuidema 1974-1976, p. 212 ; Reinhard 2002, pp. 61-72 ; Meddens et al. 2008). Choqek'iraw comporte de nombreux rochers de ce type. Ils sont souvent placés sur des terrasses et sur 
les places, dans des endroits dégagés, permettant d'observer les massifs environnants (Lecoq et Duffait 2004, p. 55), les étoiles et les constellations.

6. Constitué par trois sommets, le Cerro San Cristobal fait écho à une autre montagne de même type, le Cerro Wiracochan qui porte le nom de la divinité inca (Viracocha ou Wiraqucha) présidant à l'agriculture et à l'irrigation (Rostworowski 1983, pp. 30-39; Itier 2008, pp. 121-122). Localisé à $310^{\circ}$ au nord-ouest de Choqek'iraw, celui-ci est dominé par trois pics vers lesquels sont orientées les niches de la Maison des prêtres et les mosaïques décorées des figures de lamas. Ces deux massifs semblent avoir dessiné un axe rituel ou ceque, d'orientation nord-ouest/sud-est, que suit le cours du Río Apurimac.

7. L'Apurimac, littéralement « le grand ou le vieux seigneur qui parle ou murmure »-se décomposant en quechua en apu ou « grand seigneur ", vieux, et rimac « murmurer, parler », selon González Holguín (1989 [1608], pp. 31 et 317) -, était également le nom donné à un oracle important de la région, localisé non loin de Limatambo et incarné par une idole taillée dans un tronc d'arbre (Cieza de Léon 1984 [1553], p. 256), qui aurait pu être plus ou moins affilié à Choqek'iraw. Selon Garcilaso (1982 [1609], livre III, chap. X, p. 276), le fleuve Apurimac est aussi désigné comme le "grand fleuve » capac mayu ou capac mayta. L'anthropologue Gilda Carrera Balgas (communication personnelle, août 2009), responsable de la maison de la culture de la ville d'Abancay, précise que le terme apurimac pourrait également se traduire par «le médiateur » ou « celui qui négocie entre les hommes et les dieux ".

8. Par leur disposition, les escaliers obligent le visiteur qui les emprunte à effectuer un mouvement ondulant similaire aux méandres du fleuve placé en contrebas. Celui-ci évoque également certaines danses rituelles préhispaniques (taquis) au cours desquelles les participants se déplaçaient en zigzaguant, imitant les déplacements du serpent.

9. D'après le dictionnaire quechua-espagnol de l'Academia nacional de la lengua quechua (1995, p. 195), le terme kallanka désigne « un édifice couvert, de grandes proportions, qui servait à héberger les foules lors des célébrations de l'époque inca ". Il semble contenu dans les mots quechua callancarumi "grandes pierres travaillées, pour les fondations et les seuils de porte" et callancahuaci "grande maison érigée sur ces fondations "), voir González Holguín (1989 [1608], p. 44). C'est cette définition que reprennent Gasparini et Margolies (1977, p. 204), à partir de témoignages de chroniqueurs espagnols, en ajoutant « qu'il s'agit d'un grand édifice de plan rectangulaire allongé, recouvert d'un toit à deux pans soutenu par une série de piliers placés le long de l'axe longitudinal. L'un des côtés les plus longs est muni de plusieurs portes d'accès qui ouvrent toujours sur la place principale ».

10. La présence, dans l'une de ces structures, du squelette d'un individu, vraisemblablement de sexe féminin, orienté vers le Cerro Corihuayrachina, associé à de nombreux ossements de plusieurs camélidés, apparemment sacrifiés au moment de l'inhumation (d'après Nicolas Goepfert communication personnelle), ainsi qu'à des coquillages marins, traditionnellement liés à l'eau, pourrait peut-être indiquer que Choqek'iraw était un sanctuaire associé aux manifestations climatiques, comme le propose Bauer (2000, p. 29) pour plusieurs sites de la région de Cuzco.

11. Le terme ushmu a de multiples significations. Selon González Holguín (1989 [1608], p. 358), il désigne " le tribunal d'un juge, fait d'une pierre plantée dans le sol » ou " une borne, lorsqu'il s'agit d'une grande pierre fichée dans le sol $"$. Selon le dictionnaire quechua-espagnol de l'Academia nacional de la langue quechua (1995, p. 694), ushmu se réfère à « une petite structure de pierre ou d'un autre matériau, située le plus souvent au milieu des places des centres urbains, qui servait de trône pour les Incas ou leurs représentants, au moment de présider certaines cérémonies oflicielles ou profanes ». En ethnohistoire, ce terme renvoie « au premier huaca du cinquième ceque Payan, du secteur de l'Antisuyu. Il s'agissait d'un sanctuaire de pierre, localisé sur la place Urin Hawkaypata de l'actuelle Limatambo. Les jeunes hommes qui allaient devenir des oreillards lui faisaient des offrandes "). Zuidema (1989, p. 408), qui fut l'un des premiers chercheurs à travailler sur ce thème, le considère comme un accès à l'inframonde, capable d'absorber les offrandes liquides que l'on versait dessus lors des cérémonies. Zuidema (1989, p. 453) définit l'ushmu également comme un observatoire qui marquait le centre de la place du Cuzco et qui, conjointement avec le temple du Soleil, contribua au tracé original du plan de la ville. Selon cet auteur, il pouvait s'agir d'un bassin, d'une fontaine associée à un gnomon jouant le rôle 
d'un axis mundi, en liant les ancêtres et les huaca avec les divinités du cosmos (Zuidema, cité in Meddens et al. 2008, p. 325). Aujourd'hui, ce terme désigne tout d'abord un édifice à degrés ou une plate-forme où est construit un trône, un autel ou un lieu destiné à des personnages de haut rang (Gasparini et Margolies 1977, pp. 271-280, 356 ; Staller 2008 ; Meddens et al. 2008). Selon Protzen (1994, p. 204), le mot peut aussi désigner une plate-forme administrative et cérémonielle, en forme de dais, sur laquelle les seigneurs incas présidaient aux festivités, passaient les armées en revue ou offraient des sacrifices aux dieux. Selon le même auteur, il s'agissait, à l'origine, de la petite pierre en forme de pain de sucre, posée sur le sommet d'une plate-forme, sur laquelle on versait des liquides sacrés. Avec le temps, le terme finit par désigner la structure tout entière et un élément récurrent de l'urbanisme inca. L'ushmu pouvait également désigner, comme à Cuzco ou sur le site de Huanuco Pampa, un centre cérémoniel d'où partaient différentes lignes astronomiques (ceque) servant à l'organisation spatiale de la cité ou du site (Pino Matos 2004a et b). Enfin, l'ushmu pouvait également être perçu comme un huaca d'importance considérable qui était symboliquement lié au culte de la montagne (Kolata 1993, pp. 109-111), d'après l'hypothèse selon laquelle les montagnes visibles à l'horizon mais aussi, par analogie, les glaciers éloignés et, finalement, l'océan influent sur la météorologie de la région (Zuidema 1989, pp. 43-44). C'est ce rôle mutiple que pourrait avoir joué l'ushuu de Choqek'iraw, comme une plate-forme isolée et dressée vers le ciel, à l'aplomb du Río Apurimac et à l'emplacement de l'un de ses méandres. Depuis l'ushmu, l'Apurimac semble s'enfoncer sous la terre, du côté est, pour en émerger à l'ouest, à l'emplacement des terrasses décorées des motifs de lamas.

12. Les fouilles entreprises par Bingham, en 1911 et, plus récemment, par les archéologues de Copesco, au sommet de cette colline, ont mis au jour de petites structures, formant des motifs en damiers, construites à l'aide de petites pierres, alignées selon deux grands axes, nord-est/sud-ouest et nord-ouest/sud-est, orientés vers certains des principaux sommets environnant le site, tels le Corihuayrachina-Yanacocha au nord-est ou le Wiracochan au nord-ouest. Ces constructions auraient pu faire office d'observatoire (Plan Copesco 2003). Elles évoquent également des maquettes en forme de maison, de champs et de jardins miniatures (alasitas ou peticiones: Girault 1987, pp. 399-402) érigées, comme exvotos, par les habitants des lieux, à l'emplacement de certains centres cérémoniels liés aux montagnes environnantes, notamment au sommet du Calvaire de Copacabana ou du Cerro Baúl, à Moquegua. Ces similitudes troublantes témoignent d'une persistance d'anciens cultes, dont les traces matérielles restent encore peu étudiées par les archéologues.

13. Ce sommet, haut de $4400 \mathrm{~m}$, est également celui vers lequel sont orientées les terrasses décorées du versant occidental. Il semble faire écho au massif à trois pointes de San Cristobal, qui marque, à quelque $24 \mathrm{~km}$ au sud-est, le début de la vallée de l'Apurimac et, plus à l'est, de la vallée de Cuzco.

14. À notre avis, la présence de fusaioles à l'intérieur de ces édifices n'est pas une preuve suflisante pour en indiquer la fonction car, aujourd'hui encore, il est d'usage dans les foyers des pasteurs des hautes montagnes andines que chaque ménagère possède une douzaine de fuscaux, de différentes tailles, adaptés à l'épaisseur des fils de laine qu'elle souhaite obtenir. Les fusaïoles montrent simplement l'existence d'activités de tissage, ce qui est une caractéristique du monde rural andin.

15. Il n'existe à ce jour aucun plan général de Choqek'iraw montrant la répartition des différents secteurs. Le noyau urbain est le seul à avoir été cartographié, un plan largement diffusé dans les ouvrages relatifs à ce site.

16. Provenant des mots quechuas phaqccha ou paccha (cascade) et wayq'o (brèche ou ravine), d'après le dictionnaire de l'Academia nacional de la lengua quechua (1995).

17. Dans les Andes, les portes ou puncu en quechua (González Holguin 1989 [1608], p. 295) n'avaient pas seulement une fonction défensive ou restrictive, elles marquaient le passage d'un point ou d'un état à un autre. Ici, le passage du monde extérieur à celui de l'intérieur du complexe cérémoniel et sacré.

18. Sans entrer dans les détails et pour rester dans le sujet de ce travail, nous rappellerons que ce système était fondé sur l'observation de 41 points d'horizon ayant une importance géographique ou politique. Selon de Bock et Zuidema (1990, p. 461), « ces observations étaient effectuées depuis le Coricancha, le temple central du Soleil à Cuzco suivant des lignes imaginaires appelées ceque. Ces ceque 
étaient ponctuées de 328 huaca, peut-être 400 , selon Bauer (2000, p. 177), composés d'une vaste gamme de lieux naturels et sacrés, comme des cavernes, des sources et des sommets de montagnes, ainsi que d'autres sites construits par l'homme, reproduisant des maisons, des sources ou des canaux (ibid.) dont le nombre était déterminé par le calendrier. Les 328 huaca correspondaient à 328 jours répartis en 12 mois, équivalents à 12 mois sidéraux, soit $12 \times 271 / 3=328$, l'année solaire comportant, elle, une période [supplémentaire] de 37 jours. Les 328 jours, liés à la durée de visibilité des Pléiades, se comptaient à partir de la nuit du 8 au 9 juin jusqu'à celle du 3 au 4 mai de l'année suivante et marquaient une saison agricole. La période [supplémentaire] de 37 jours était comprise entre la récolte et les préparatifs pour une nouvelle saison. Cette période est également considérée comme transitoire entre deux saisons ». Sur ce sujet, le lecteur pourra également consulter d'autres travaux de Zuidema (1974-1976 ; 1978 ; 1995, chap. I ; 2008b). La montagne de Choque Quirao mentionnée par Zuidema appartenait à un groupe de huaca localisé près du lieu-dit Chuquicancha, «la Maison du Soleil », le plus éloigné de Cuzco, qui se trouvait sur le ceque du solstice de juin.

19. Bauer (2000, p. 94) indique, en effet, que «Chuquiquirao Puquio a été décrit comme une source, localisée dans une chaîne de montagne de Cariurco ", où se trouvent " plusieurs édifices, des pierres taillées dans le plus pur style inca, deux petites montagnes aménagées en terrasses et une petite carrière ». Mais la localisation de ce site porte à confusion, car il semble se confondre avec celui d'Aucapuqui qui constitue le cinquième huaca de ce ceque. De plus, comme le fait remarquer Saintenoy (communication personnelle) qui a visité le site de Cuzco et rédigé une thèse sur Choqek'iraw et les établissements incas et préincas de la vallée de l'Apurimac, Choqek'iraw Pukio se situe sur le quatrième ceque dont les sept huaca sont liés à Awqaylli Panaka, le lignage de Yawar Qapaq, alors que le site de Choqek'iraw qui nous occupe ici est un patrimoine des lignages de Pachakuti ou de Tupaq Yupanki. Selon cet auteur, les deux sites n'appartiennent pas non plus à la même catégorie : le premier est un huaca de Cuzco intégré au paysage de la capitale, alors que le second est un site isolé dans la cordillère de Vilcabamba qui ne semble pas appartenir au système des ceque de l'Antisuyu, tel que présenté par Zuidema (1978) et Bauer (2000). Il est vrai que, comme le souligne à nouveau Bauer (2000, p. 36), « la plupart des sites incas [les plus connus de la région] de Cuzco [comme la majorité des roches taillées de la zone de Sacsahuaman] ne figurent pas dans la liste des sanctuaires établie par Cobo ». On peut donc se demander ce qu'il en est des sites cérémoniels localisés dans les régions plus éloignées de la capitale inca, comme Choqek'iraw. Il est probable que ce site appartenait à un autre système de ceque régional, similaire à celui de la région d'Anta (à l'est de Choqek'iraw), étudié par Bauer (ibid.), dont les huaca portaient le même nom que ceux de la capitale, système qui se superposait apparemment à celui, beaucoup plus complexe, du Cuzco. Molinié (1996) avait identifié, dans la vallée de Yucay, la présence de plusieurs huaca, dont les toponymes reprenaient les noms de certains huaca du système de ceque de Cuzco, ce qui, pour l'auteur, n'avait rien d'étonnant puisque Yucay était considéré comme une extension de la cité de Cuzco.

20. Ces derniers sites sont aussi connus sous les noms de Pajatén, Gran Pajatén ou Yaro.

21. Le fait de retrouver des motifs décoratifs de ce type sur les façades de certains édifices chachapoya, à Kuelap, a été interprété, par plusieurs archéologues de Copesco, comme le témoignage de possibles contacts entre les deux régions ou la présence de colons (mitimae) chachapoya à Choqek'iraw. Toutefois, aucun document ne confirme que des populations chachapoya résidaient effectivement à Choqek'iraw à cette époque, et les techniques décoratives sont différentes sur les deux sites.

22. Ces altitudes restent toutefois imprécises, en raison de la mauvaise couverture des satellites sur le site et des accidents du relief qui perturbent les signaux GPS. Elles donnent néanmoins une idée approximative de l'emplacement et de l'étagement des figures sur les terrasses.

23. Les dessins de la Figure 7 reposent sur des photographies prises à la fois au moment de leur découverte et lors de notre dernière visite sur le site en août 2009. La reproduction des lamas des terrasses supérieures reste approximative et s'appuie sur des clichés pris au téléobjectif, car le secteur était alors en cours de réaménagement et inaccessible sans une autorisation préalable des responsables de Copesco, mais ils ont été vérifiés ensuite grâce aux croquis reproduits dans le rapport remis à l'INC par les responsables de la restauration du site. 
24. Lors des voyages de troc à travers les Andes, il est d'usage que l'animal de tête, le yaso ou punta delantero (ou les deux autres animaux qui l'accompagnent, le chawpi et chaca delantero) qui guide(nt) la troupe, arbore(nt) des décorations - colliers, cloches de bronze, sac à coca (cluspa) - hautement symboliques, similaires à celles reproduites sur la Figure $10 \mathrm{~d}$ et e, et représentées sur les mosaĩques. Les cloches sont destinées à effrayer les animaux errants, comme les renards qui pourraient inquiéter les lamas et provoquer la débandade de la troupe. Une fonction rituelle peut être rattachée à la puissance fécondante du son (Lecoq et Fidel $2000 ; 2003$ ). Les décorations florales multicolores ( $t$ 'ika) qui ornent leurs oreilles leur sont remises au moment du Carnaval et visent à montrer l'union des animaux avec la Terre Mère et les sources qui les ont enfantés. Chaque couleur joue un rôle codifié déterminant (Flores Ochoa 1974-1976 ; Brougère 1984 ; Flores Ochoa et al. 1995; Lecoq et Fidel 2003).

25. Aucun indice ne permet de déterminer le sexe de ces animaux, mais, par analogie avec les caravanes actuelles, on peut penser qu'il s'agit de mâles. En effet, la plupart des grandes caravanes de troc qui parcourent les Andes à la saison sèche se composent de lamas mâles, âgés de deux à sept ans. Les plus jeunes ne portent pas de charge, mais suivent le reste de la troupe pour s'habituer à l'itinéraire. Tous sont castrés, afin de leur donner plus de force (Lecoq 1987). Quelques animaux non castrés restent dans les pâturages d'altitude, afin d'assurer la reproduction du cheptel. Il s'agit en général d'un animal reproducteur pour huit à dix femelles. Ce sont ces animaux qui pourraient être représentés ici sur les terrasses de la partie haute $\mathrm{n}^{\circ} 1$ à 4 .

26. Cette roche, localisée au milieu de la terrasse, pourrait avoir joué une fonction rituelle, similaire à celle que nous avons déjà signalée pour les roches dispersées à travers le site.

27. En guise de comparaison, l'ile de Taquile, située au cœur du lac Titicaca, offre un exemple intéressant d'organisation de l'espace et des rituels obéissant au même modèle : il pourrait nous aider à mieux comprendre l'organisation des motifs de Choqek'iraw. Selon Bellenger (2007, pp. 117, 221, 222), Taquile est divisée en six suyu et terrains de cultures, trois au nord et trois au sud. Chacun regroupe un calvaire faisant office de sanctuaire à un esprit tutélaire; deux sont considérés comme les lieux de résidence des ancêtres fondateurs de l'ile, l'un féminin au sud, l'autre masculin au nord. Les campo warayuq ou curaca locaux en effectuent une visite annuelle par groupe de trois, selon trois itinéraires préalablement établis par les responsables communautaires de chaque moitié. Ils se réunissent ensuite dans la maison de l'un des membres de la communauté. "Le cycle complet des réunions de chaque membre s'étale sur deux mois, dans dix-huit lieux différents " (ibid., p. 227). Sur le même thème, Molinié (1996) indiquait que, dans la vallée de Yucay, la répartition des sept calvaires de la région renvoyait aux sept anciens ayllu qui composaient la communauté avant qu'elle ne soit réorganisée en deux moitiés.

28. Pour le moment, aucun indice ne permet réellement de démontrer que ces terrasses ont effectivement été mises en culture, si ce n'est la présence de la source, aujourd'hui asséchée, et de plusieurs canaux d'irrigation. Les quelques prélèvements effectués lors de leur restauration par les archéologues de Copesco et étudiés par les biologistes du projet (Pumaccahua Rocca 2005) ont fourni des macro-restes et du pollen de maïs, de coca et de haricot qui auraient pu provenir de vallées voisines où poussaient la coca et ce type de plantes. Pour ce qui est de la coca, il pourrait également s'agir d'anciennes offrandes puisque, dans le Pérou préhispanique, aux époques de labourage, des semailles et de la récolte, on répandait de la coca et de la chicha dans les champs, tout en sacrifiant des lamas (Karsten 1957, p. 206). Aujourd'hui encore, lors de certaines cérémonies pastorales liées à la fertilité et à la reproduction des troupeaux, il est d'usage d'offrir à la Terre Mère des feuilles et des graines de coca symbolisant les pâturages où paissent les animaux, et notamment les mâles reproducteurs, parce que la coca conserve sa couleur verte très longtemps qui évoque celle des prairies d'altitude (Flores Ochoa 1974-1976, p. 255). Il convient également de noter que le site de Choqek'iraw a été incendié à plusieurs reprises au cours de son histoire, ce qui limite nos chances d'obtenir des données fiables quant aux plantes qui y étaient effectivement cultivées à la période inca.

29. Toutes les citations en langue étrangère ont été traduites ici par l'auteur, PL.

30. La restauration des motifs et le traitement chimique des pierres qui les composent ont certainement détruit les traces qui auraient pu subsister. 
31. L'unku est le vêtement principal de l'homme andin. Il s'agit d'une pièce rectangulaire ou carrée, disposant d'une ouverture dans la partie centrale pour passer la tête - comme les ponchos - qui était ensuite unie de chaque côté en laissant des ouvertures afin de passer les bras (Desrosiers 1992, p. 12 ; Gisbert et al. 1987, pp. 59-67).

32. Des coupes en bois ou keru, qui avaient servi à effectuer des libations lors des funérailles, étaient également insérées dans la façade de l'édifice, afin de matérialiser symboliquement les yeux du mort par lesquels il pouvait ainsi communiquer avec le monde des vivants lors de certaines cérémonies (Abercrombie 1998, p. 182).

33. Les Espagnols n'éradiquent pas pour autant l'usage du textile à des fins cérémonielles, mais en détournent le sens à leur profit. Ainsi, la grande mine d'argent du Cerro Rico, à Potosi, assimilée à la Terre Mère, la Pacha Mama, prend l'apparence d'une vierge revêtue d'une somptueuse robe dans le tableau colonial peint par González Holguín, aujourd'hui conservé au musée de la Casa de la Moneda de la cité impériale, reproduit par Gisbert (1980, pp. 82-84, illustration 106 ; Gisbert et al. 1987, p. 318, fig. 1). De nombreux éléments et divinités préhispaniques, comme le Soleil, le Singe, la Sirène présents dans les décors architecturaux andins, depuis Arequipa jusqu'à Potosi, se retrouvent dans les tapisseries du XVIII ${ }^{e}$ siècle (Gisbert et al. 1987, pp. 312-318).

34. Pour González Holguín (1989 [1608], p. 345) le terme tucapu désigne « les vêtements précieux au crochet ou les draperies tissées au crochet ». Il s'agit de petits cadres carrés renfermant des figures géométriques, souvent placés le long de la partie basse des tuniques masculines et des robes féminines (Itier 2008, p. 152).

35. Dans la tradition andine, les cervidés, comme les vigognes ou les renards, sont directement liés au monde non domestiqué des montagnes et des Apu (Molinié 1996, p. 211 ; Walter 2003; Goepfert 2009). Le fait de figurer des cervidés dans la partie supérieure du sac à coca, en association avec la Croix du Sud, pourrait être une évocation du monde complexe des pasteurs et de leurs divinités agrestes tutélaires.

36. C'est ce que suggèrerait également la présence de cervidés, sur le sac conservé au musée d'Ica.

37. On appelle illa les petites sculptures en forme d'alpaca, de lama. Ces représentations d'animaux jouent un rôle important dans les cérémonies propitiatoires (Flores Ochoa 1974-1976). En quechua, le terme illa désigne également des petites pierres et des bézoards, concrétions formées par des poils ou débris végétaux dans le corps de certains ruminants, que les populations des Andes conservaient en gage de prospérité (González Holguín 1989 [1608], p. 367 ; Duviols 1974-1976, p. 283). Nous avons vu que le terme illa renvoie également à la lumière car yllarini signifie resplendir, étinceler, briller (González Holguín 1989 [1608], p. 367). Illapa est aussi la divinité de l'éclair et de la foudre (Rostworowski 1983, pp. 39-42 ; Urton 2006, p. 99) qui est désignée par Chhoqqueylla en quechua (González Holguín 1989 [1608], p. 116), terme à nouveau peut-être en rapport avec le nom du site de Choqek'iraw. Toutes ces notions expriment bien la même idée de fertilité et de fécondation des troupeaux, liée au cycle des saisons.

38. LLoqsimushan n'apparaît pas dans les dictionnaires quechuas que nous avons consultés. Il serait composé des termes: llocssini qui, selon González Holguín (1989 [1608], p. 215) signifie « sortir à l'extérieur » et muchhani, "égrener le maïs » (ibid., p. 246). Hatun inti signifierait le Grand soleil, Hatun signifiant « le grand, le mieux, ou supérieur, le plus important, le mieux connu » (ibid., p. 154). Inti Chinka Pushan ou le soleil couchant est à rapprocher des mots chincariptui qui signifie " disparaître, s'absenter » et ppunchau, « le jour et le soleil », ppuchao yanni « le soleil au haut du ciel, en plein jour » (ibid.).

39. Comme le précisent ces auteurs, et sans entrer dans le détail, les données relatives à ces constellations sont confuses, car il semble que les chroniqueurs espagnols aient mal perçu les informations qu'ils ont recueillies. La constellation du Lama est de fait désignée sous plusieurs noms, notamment ceux d'Urcuchillay et Catachillay qui désigneraient un grand lama aux multiples couleurs, chargé de préserver les troupeaux, mais aussi son petit. Choquechinchay serait, pour González Holguín (1989 [1608], p. 117), une représentation du lama céleste, alors que, pour les autres chroniqueurs, il serait l'incarnation d'un grand fauve, protecteur de tous les félins et des ours. Selon Bouysse-Cassagne (2004, pp. 75-80), il symboliserait un aspect du soleil nocturne et serait lié à l'inframonde et aux mines. 
40. Aujourd'hui encore, pour les populations pastorales des hauts plateaux boliviens que nous avons côtoyées, la Voie Lactée est décrite comme un fleuve sacré et un lieu d'origine ou pacarina, peuplé d'animaux divers et de personnages mythiques, dont un grand lama, l'Urcuchillay ou Chhoque Chinchay, et son petit, Catuchillay, tous deux menés par un berger, Micchic et par son fils, Katheki, brandissant leur fronde céleste. On y trouve également un serpent, Machacuay, une perdrix, Yutu, un renard, Atoq, ainsi qu’un crapaud, Hamp'ata. Les Pléiades ou collqa (" greniers ») y jouent également un rôle déterminant, car elles apparaissent dans le ciel au moment des moissons et servent à réguler l'année agricole.

41. Offrandes composées à partir de trois feuilles de coca.

42. D'une certaine façon, on pourrait assimiler ces ballots aux fardos funéraires qui, dans de nombreuses cultures péruviennes, enveloppaient les morts. Ils jouent le même rôle de contenant rituel et se rapprochent en cela des q'epi ou q'ipi de la région de Coroma, en Bolivie, utilisés pour garder les textiles sacrés de la communauté et qui incarnent les esprits des ancêtres protecteurs tout en délimitant l'espace communautaire (Bubba 1997).

43. Ainsi, l'usage d'accrocher des objets miniatures symboliques (lamas en farine de quinua, crapauds séchés ou en argent, fiûtes de pan, pièce de monnaie et autres billets miniatures factices), sur une pièce d'étoffe évoquant une 'unkhuña, en guise de bon augure, est encore bien répandu dans les communautés rurales de Bolivie, notamment lors de la fête des miniatures ou alasitas, qui se tient à la Paz tous les 24 janvier (Ponce Sangines 1969).

\section{RÉFÉRENCES CITÉES}

\section{ABERCROMBIE Tom}

1998 Pathways of memory and power, The University of Wisconsin Press, coll. " Ethnography and History among an Andean People », Madison.

Absi Pascale

2003 Les ministres du diable. Le travail et ses représentations dans les mines de Potosi, Bolivie, L'Harmattan, Paris.

Academia nacional de la lengua quechua

1995 Diccionario Quechua-Español-Quechua, Municipalidad del Qosqo, Qosqo.

Alencastre Montufar Gustavo

2006 "Choquequirao », Boletín de Lima, XXVII (146), pp. 62-72.

ANGRAND Leonce

1972 Imagen del Perú en el siglo xIx, Carlos Milla Batres edit., Lima.

Arnold Denise et Juan de Dios YaPITA

1992 "En el corazón de la plaza tejida : el wayñu en Qaqaxchaka », $\sigma^{a}$ Reunión anual de etnología, Museo de Etnografía y Folklore, La Paz, pp. 17-70.

BaCa Cosio Jenaro Fernando

1989 Motivos de ornamentación de la cerámica inca Cuzco, tomo II, Editorial Navarete, Lima.

BAUER Brian

2000 El espacio sagrado de los incas. El sistema de ceque del Cuzco, Centro Bartolomé de las Casas, coll. " Archivos de Historia Andina » 33, Cuzco.

Bauer Brian S. et David S. P. Dearborn

1998 Astronomía e Imperio en los Andes, Centro Bartolomé de las Casas, Cuzco. 


\section{Bauer Brian S. et Charles Stanish}

2003 Las islas del Sol y de la Luna. Ritual y perigrinación en el lago Titicaca, Centro Bartolomé de las Casas, Cuzco.

BELLENGER Xavier

2007 El espacio musical andino. Modo ritualizado de producción musical en la isla Taquile y en la región del lago Titicaca, Instituto francés de estudio andinos/ Centro Bartolomé de las Casas/Pontificia Universidad Católica del Perú/ Embajada de Francia/IRD, Lima.

\section{BERENGUer José}

1999 «El evanescente lenguaje del arte rupestre en los Andes atacameños », in José Berenguer Rodríguez y Francisco Gallardo Ibañez (éds), Arte rupestre en los Andes del Capricornio, Museo Chileno de Arte Precolombino, Banco de Santiago, Santiago, pp. 9-56.

\section{BERTHELOT Jean}

1978 "L'exploitation des métaux précieux au temps des Incas », Annales. Économies, Sociétés, Civilisations, 5-6, pp. 948-966.

BERTONIO Ludovico

1984 Vocabulario de la lengua aymará, Ceres/IFEA/Musef, La Paz [1612].

BingHAM Hiram

1910 "The ruins of Choqquequirau », American Anthropologist, 12, pp. 505-525. 2006 "Choqquequirau », Boletin de Lima, XXVII (146), pp. 52-61.

Bock Edward K. de et Tom R. Zuidema

1990 "Cohérence mathématique dans l'art andin », in Inca-Perú. 3000 ans d'Histoire, musée royaux d'Art et d'Histoire, Bruxelles, pp. 460-469.

Bonavia Duccio.

1974 Ricchata Quellccani, pinturas murales prehispánicas, Fondo del Libro del Banco Industrial del Perú, Lima.

1990a "Peinture murale au Pérou », in Inca-Perú. 3000 ans d'Histoire, musée royaux d'Art et d'Histoire, Bruxelles, pp. 412-423.

1990b "Les peintures murales dans l'ancien Pérou », L'Anthropologie, 94 (1), pp. 155-170.

1990c "Les ruines de l'Abiceo », in Inca-Perú. 3000 ans d'Histoire, musée royaux d'Art et d'Histoire, Bruxelles, pp. 248-261.

1996 Los camélidos sudamericanos. Una introducción a su estudio, IFEA/UPCH/Conservation International, Lima.

BOUCHARD Jean-François

1983 Contribution à l'étude de l'architecture inca : établissements de la vallée du Rio Vilcanota-Urubamba, Éditions de la Maison des Sciences de l'Homme, Paris.

BOURGet Steve

1990 «Des tubercules pour la mort. Analyses préliminaires des relations entre l'ordre naturel et l'ordre culturel dans l'iconographie mochica ", Bulletin de l'Institut français d'études andines, 19 (1), pp. 45-85. 
Bouysse-Cassagne Thérèse

1978 «L'espace aymara : urco et uma », Annales. Économies, Sociétés, Civilisations, 5-5, pp. 1057-1080.

1987 La identidad aymara, Hisbol, La Paz.

1997a «De Empédocles a Tunupa: evangelización, hagiografía y mitos», in Thérèse Bouysse-Cassagne (éd.), Saberes y Memorias. In memoriam Thierry Saignes, Credal, Paris/IFEA, Lima, pp. 157-212.

1997b «Le palenquin d'argent de l'Inca : petite enquête d'ethno-histoire à propos d'un objet absent ", Techniques et Cultures, 29, pp. 69-111.

1998 «Attention! Un diable peut toujours en cacher un autre: à propos de l'introduction des images de l'Enfer chez les Indiens de l'Altiplano bolivien ", Trace, 34, pp. 22-40.

2004 «El Sol de adentro: wakas y santos en las minas de Charcas y en el lago Titicaca, siglos Xı a Xvı », Boletín de Arqueología PUCP, 8, Lima, pp. 59-97.

2005 «Las minas del centro-sur andino, los cultos prehispánicos y los cultos cristianos ", Bulletin de l'Institut français d'études andines, 34 (3), pp. 443-462.

\section{BOUYSSE-CASSAgne Thérèse et Olivia Harris}

1987 "Pacha : en torno al pensamiento andino ", in Thérèse Bouysse-Cassagne, Olivia Harris, Tristan Platt et Verónica Cereceda (éds), Tres reflexiones sobre el pensamiento andino, Hisbol, La Paz, pp. 11-59.

\section{BRAY Tamara}

2008 "Las dimensiones simbólicas del poder dentro del imperio inca », in Paola González Carvajal et Tamara L. Bray (éds), Lenguajes. Visuales de los incas, British Archeological Report, $\mathrm{n}^{\circ}$ 1848, Oxford, pp. 13-19.

Brougere Anne-Marie

1984 "Stratégie d'échange et relations de marché : le cas de Sibayo », Bulletin de l'Institut français d'études andines, XIII (1-2), pp. 63-79.

1988 "El t'inkachiy : revitalización simbólica del enqa en la puna de Arequipa », Antropológica, 6, Lima, pp. 67-83.

Browman David

1988 «Llama caravan fleteros and their importance in the production and distribution ", in Philip C. Salzman et John G. Galaty (éds), Nomads in a changing world, Instituto Universitario Oriente di Napoli, Napoli, pp. 317-370.

\section{BubBa Cristina}

1997 «Los rituales a los vestidos de María Títiqhawa, Juana Palla y otros fundadores de los ayllu de Coroma ", in Thérèse Bouysse-Cassagne (éd.), Saberes y Memorias. In memoriam Thierry Saignes, Credal, Paris/IFEA, Lima, pp. $377-400$.

\section{Campana Cristobal}

2006 Chan Chan del Chimo. Estudio de la ciudad de adobe más grande de América antigua, Editorial ORUS, Lima. 


\section{Cereceda Verónica}

1978 «Sémiologie des tissus andins : les talegas d'Islugas », Annales. Économie, Société et Civilisation, 5, pp. 1017-1036.

1987 "Aproximaciones a una estética andina : de la belleza al tinku », in Thérèse Bouysse-Cassagne, Olivia Harris, Tristan Platt et Verónica Cereceda (éds), Tres reflexiones sobre el pensamiento andino, Hisbol, La Paz, pp. 133-231.

\section{Ceruti Constanza}

1999 Cumbres sagradas del Noroeste argentino, Eudeba, Buenos Aires.

Chacama Juan (éd.)

2008 Arica. Cultura milenaria, catalogue de l'exposition, 26 de agosto al 12 de octubre 2008, Centro cultural Palacio La Moneda/Museo Universidad de Tarapacá, Santiago.

\section{Cieza De Leon Pedro}

1984 Crónica del Perú, Primera parte, Pontificia Universidad Católica del Perú/Academia Nacional de la Historia, Lima [1553].

\section{Сово Bernabé}

1964 Historia del muevo mundo, Atlas, Biblioteca de Autores Españoles, XCI-XCII, Madrid [1653].

\section{Custred Glynn}

1974 " Llameros y comercio interregional », in Giorgio Alberti et Enrique Mayer (éd.), Reciprocidad e intercambio en los Andes Peruanos, Instituto de Estudios Peruanos, Lima, pp. 252-289.

D'Altroy Terence

2005 The Incas, Blackwell Publishing, Malden, MA/Oxford.

Descola Philippe

2005 Par-delà nature et culture, Éditions Gallimard, NRF, Paris.

\section{DESROSIERS Sophie}

1992 "Las técnicas de tejido ¿ tienen un sentido ? Una propuesta de lectura de los tejidos andinos ", Revista Andina, 1, Cuzco, pp. 7-46.

1997 "Lógicas textiles y lógicas culturales en los Andes », in Thérèse BouysseCassagne (éd.), Saberes y Memorias. In memoriam Thierry Saignes, Credal, Paris/IFEA, Lima, pp. 325-349.

D'Harcourt Raoul

2008 Les textiles anciens du Pérou et leurs techniques, musée du quai Branly/Flammarion, Paris [1934].

\section{Drake Moraga Vanesa}

2005 Animal myth and magic : images from Pre-Columbian textiles, Ololo Press, Larkspur, CA.

DuFfait Erwan

2005 "Choqequirao en el siglo Xvı : etnohistoria e implicaciones arqueológicas », Bulletin de l'Institut français d'études andines, 34 (2), pp. 185-196. 
Le réseau routier inca de la cordillère de Vilcabamba (département de Cuzco, Pérou) : description, organisation, origines et fonctions, thèse de doctorat, Université Paris 1, Paris.

Duviols Pierre

1974-1976 « Une petite chronique retrouvée : errores, ritos, supersticiones y ceremonias de los yndios de la pouincia de Chinchaycocha y otras del Piru », Journal de la Société des Américanistes, LXIII, pp. 275-297.

Echevarría López Gori Tumi

2005 "Investigación de los motivos decorativos del sector VIII », in Zenovio Valencia García, Helguero Gonzáles, Jorge Chávez Rodríguez, César Morante Ángeles et Abdias Calsin Ríos (éds), Investigacion histórica del complejo histórico de Choqequirao, Sector VIII, Andenes de las lamas, Proyecto Plan Copesco Nacional, Informe final, Cuzco, pp. 194-269.

2008 Choquequirao. Un estudio arqueológico de su arte figurativo, Hipocampo Editores, Lima.

FLoRES OCHOA Jorge

197-1976 «Enqa, Enqaychu, Illa y Khuya Rumi : aspectos mágicos-religiosos entre pastores ", Journal de la Société des Américanistes, LXIII, pp. 245-262.

1975 "Pastores de alpacas ", Allpanchis, 8 (VIII), Cuzco, pp. 5-23.

1978 "Classification et dénomination des camélidés sud-américains », Annales. Économies, Sociétés, Civilisation, 5-6, pp. 1006-1015.

1998 Qeros. Arte inka en los vasos ceremoniales, Banco de Crédito del Perú, Lima.

Flores Ochoa Jorge, Kim Macquarrie et Javier Porus

1995 Oro de los Andes, Las llamas, alpacas, vicuñas y guanacos de Sudamérica, 2 tomes, Jorsi Blassi éditions, Barcelone.

FRAME Mary

1994 « Las imágenes visuales de estructuras textiles en el arte del antiguo Perú », Revista Andina, 2, Cuzco, pp. 295-372.

2007 "Lo que Guaman Poma nos muestra, pero no nos dice sobre Tukapu ", Revista Andina, 44, Cuzco, pp. 9-69.

Franco Jordan Régulo et Juan Vilela Puelles

2005 El Brujo. El mundo mágico religioso mochica y el calendario ceremonial, Minka, Trujillo.

Franquemont Edward, Christine Franguemont et Billie Jean Isbell

1992 «Awaq ñawin : el ojo del tejedor. La práctica de la cultura en el tejido », Revista Andina, 1, Cuzco, pp. 47-77.

Garcilaso De La Vega Inca

1982 Commentaires royaux sur le Pérou des Incas, 3 volumes, Maspero, Paris [1609].

\section{GaSPARINI Graziano et Luise Margolies}

1977 Arquitectura inka, Centro de investigaciones históricas y estéticas/Facultad de Arquitectura y Urbanismo, Universidad Central de Venezuela, Caracas. 


\section{GisBert Teresa}

1980 Iconografia y mitos indigenas en el arte, Gisbert y Cia libreros editores, La Paz.

2000 "Les nécropoles des hauts plateaux », Archéologia, 363, pp. 42-47.

Gisbert Teresa, Silvia Arze et Marta Cajías

1987 Arte textil y mundo andino, Gisbert y Cia libreros editores, La Paz.

Gisbert Teresa, Juan Carlos Jemio et Roberto Montero

1994 "El señorio de los Carangas y los chullpares del río Lauca », Revista Andina, 2, Cuzco, pp. 427-481.

1996 «Los chullapares del río Lauca », Revista de la Academia Nacional de Ciencias de Bolivia, 70, La Paz, 82 p.

Girault Louis

1987 Rituales en las regiones andinas de Bolivia y Perú, Ceres/Musef/Quipus, La Paz.

\section{GOEPFERT Nicolas}

2009 Rites funéraires, offrandes et sacrifice animal dans l'idéologie religieuse de la culture mochica (100-800), côte nord du Pérou, thèse de doctorat, Université Paris 1, Paris.

\section{González Holguín Diego Fray}

1989 Vocabulario de la lengua general de todo el Perú llamada Lengua Qquichua o del Inca, Universidad nacional Mayor de San Marcos, Lima [1608].

Guaman Poma Felipe

$1936 \quad$ Nueva corónica y buen gobierno, Institut d'Ethnologie, Paris [1615].

\section{HARRIS Olivia}

1983 «Los muertos y los diablos entre los laymi de Bolivia », Chungará. Revista de Antropología Chilena, 11, Arica, pp. 135-152.

Hoceuenghem Anne-Marie

1987 Iconografia mochica, Pontificia Universidad Católica del Perú/Fondo editorial, Lima.

Horta Tricallotis Helena et Carolina AgüEro Pıwonka

2009 "Estilo, iconografía y función de las Inkuñas prehispánicas del norte de Chile durante el periodo Intermedio Tardío (1000-1470 d.C.) », Chungará.

Revista de Antropologia Chilena, 41 (2), Arica, pp. 183-227.

\section{ITIER César}

2008 Les Incas, Société d'édition Les Belles Lettres, Guide «Belles Lettres des civilisations ", 214, Paris.

INC (Instituto nacional de cultura del Peru)

2006 "Descripción testimonial de una faja calendario "Wata Yupana" », Taquile y su arte textil, INC, Lima, pp. 44-58.

\section{Karadimas Dimitri}

1999 «La constellation des quatre singes. Interprétation ethnoarchéoastronomique des motifs de "el Carchi-Capuli", Colombie, Équateur ", Journal de la Société des Américanistes, 85, pp. 115-145. 
KARSTEN Rafael

1957 La civilisation de l'empire Inca. Un État totalitariste du passé, Payot, Paris.

Kauffmann Doig Federico

2002 "La culture Chachapoya », Historia del Perú Antiguo, 5 (VIII), Lima, pp. 513-575.

\section{Kolata Alan}

1993 The Tiwanaku. Portrait of an Andean Civilization, Blackwell, The People of America, Cambridge/Oxford.

LARA Jesús

2001 Diccionario Qheshwa-Castellano, Los Amigos del Libro, La Paz.

LaVAllée Danièle et Luis Guillermo Lumbreras

1985 Les Andes, de la Préhistoire aux Incas, Gallimard, L'Univers des Formes, Paris.

LeCOQ Patrice

1987 "Caravanes de lamas, sel et échanges dans une communauté de Potosi, Bolivie ", Bulletin de l'Institut français d'études andines, 16 (3-4), pp. 1-38.

2004 "Choqek'iraw, un site formatif? Résultats préliminaires de la campagne de fouilles menées sur ce site en août 2004 ", Bulletin de l'Institut français d'études andines, 33 (2), pp. 379-383.

2005 "À la redécouverte de Choqequirao ", in ChoqeQuirao, El misterio de las llamas del Sol y el culto a los Apus, Fondo Controvalor Perú-Francia, Lima, pp. 69-90.

2007 "Choqek'iraw : la merveille inca des Andes ", Archéologia, 444, pp. 20-35.

2008 «Nouvelles données sur l'histoire précolombienne du site inca de Choqek'iraw, au Pérou ", Nouvelles de l'archéologie, 111-112, pp. 122-128.

LeCoQ Patrice et Ricardo Cespedes

1997 «Panorama archéologique des zones méridionales de Bolivie (sud-est de Potosi) ", Bulletin de l'Institut français d'études andines, 26 (1), pp. 21-51.

LeCoQ Patrice et Erwan DuFfaIt

2004 "Choqek'iraw au Pérou. Un nouveau Machu Picchu», Archéologia, 411 , pp. 50-63.

LECOQ Patrice et Sergio FideL

2000 "Algunos aspectos de la vida y de los ritos ganaderos en Ventilla, una comunidad pastoril del sur de Potosí, Bolivia ", in Jorge Flores Ochoa et Yoshiki Kobayashi (éds), Pastoreo altoandino. Realidad, sacralidad y posibilidades, Plural editores CID/Museo de Etnografia y Folklore, La Paz, pp. 150-183.

2003 «Prendas simbólicas de camélidos y ritos agropastorales en el sur de Bolivia ", Textos Antropológicos, 14 (1), La Paz, pp. 7-54.

LEICHT Hermann

1960 Pre-inca art and culture, Macgibbon \& Kee, London.

LOPEZ Jaime, Willer Flores et Catherine Letourneux

1993 Laymi, Salta, Pac-Potosi/Ruralter, La Paz. 


\section{LOZA-BALSA Gregorio}

1995 Astronomía y calendario aymará, Museo Nacional de etnografía y Folklore, La Paz.

\section{LUMBRERAS Luis Guillermo}

1977 "Introducción al arte textil », in Luis Guillermo Lumbreras (éd.), Arte precolombino, Museo nacional de antropología y arqueología/Banco de Crédito del Perú, Lima, pp. 8-22.

2005 «La arqueología de Choqequirao », in Choquequirao. El Misterio de las Llamas del Sol y el Culto a los Apus, Fondo Contravalor Perú-Francia, Lima, pp. 127-146.

Lumbreras Luis Guillermo, Renzo Uccelli et Walter H. Wust

2001 Choqequirao. Sanctuario histórico y ecológico, Peisa, Lima.

MaKowsKi Krzysztof

2000 «Las divinidades en la iconografía mochica », in Krzysztof Makowski (éd.), Los Dioses del antiguo Perú, Banco de Crédito del Perú, Lima, pp. 137-173.

MEDDENS Frank

2006 "Rocks in the landscape : managing the Inka agricultural cycle ", The Antiquaries Journal, 86, pp. 36-65.

Meddens Frank M., Nicholas P. Branch, Cirilo Vivanco Pomacanchari, Naomi RIDDIFORD et Rob KEMP

2008 "High altitude Ushnu platforms in the department of Ayacucho, Peru: structure, ancestors and animating essences ", in John E. Staller (éd.), PreColumbian landscapes of creation and origin, Springer Science and Business Media, LLC, Londres, pp. 315-355.

Merlino Rodolfo J. et Mario A. RabeY

1983 «Pastores del altiplano andino meridional : religiosidad, territorio y equilibrio ecológico », Allpanchis Phuturinqa, XVIII (21), Cuzco, pp. 149-170.

\section{Molina Critóbal}

1989 Fábulas y mitos de los Incas, Enrique Urbano edit., coll. « Historia » 16, Madrid [1574].

Molinié Antoinette

1996 "The spell of Yucay : a symbolic structure in Incaic terraces », Journal of the Steward Anthropological Society, 24 (1-2), Urbana, pp. 203-230.

MORRIS Craig

1967 Storage in Tawantinsuyu, PhD dissertation, The University of Chicago, Chicago.

MURRA John Victor

1975a "Maíz, tubérculos y ritos agrícolas », in John Victor Murra (éd.), Formaciones económicas y politicas del mundo andino, Instituto de Estudios Peruanos, Lima, pp. 45-57 [1960]. 
1975b «La funcción de los tejidos en varios contextos sociales y políticos », in John Victor Murra (éd.), Formaciones económicas y politicas del mundo andino, Instituto de Estudios Andinos, Lima, pp. 145-170.

\section{Palacios Rios Felix}

2000 "El simbolismo de las alpacas: ritual y cosmovisión andina ", in Jorge Flores Ochoa et Yoshiki Kobayachi (éds), Pastoreo altoandino. Realidad, sacralidad y posibilidades, Plural/Musef, La Paz, pp. 189-199.

\section{PARejo-Coudert Raphaël}

2001 "La flûte pinkuyllu des Provincias Altas du Cuzco (Pérou) : organologie et symbolisme érotique d'un aérophone andin ", Journal de la Société des Américanistes, 87, pp. 211-264.

\section{PÄRSSINEN Marti}

1992 Tawantinsuyu. The Inca state and its political organization, SHS, Helsinki.

\section{PAZ Flores Percy}

2007 "Las llamas de la koka. Esculturas en andenes de Choquequira », Actas del Primer Simposio Nacional de Arte Rupestre (Cuzco, noviembre 2004), Actas y memorias 12, IFEA, Embajada de Alemania en el Perú, Lima, pp. 237-255.

Pino Matos José Luis

2004a «Observatorios y alineamientos astronómicos en el Tampu inka de Huánuco Pampa ", Arqueología y Sociedad, 15, Lima, pp. 173-190.

2004b «El ushmu y la organización del espacio en los principales tampus de los wamani de la sierra central del Chinchaysuyu », Chungará. Revista de antropología chilena, 36, Arica, pp. 303-311.

\section{Plan Copesco}

2003 Implementación del plan maestro Choquequirao, Expediente técnico, Cuzco.

Platt Tristan

1978 "Symétries en miroir. Le concept de yanantin chez les Macha de Bolivie ", Annales. Économies, Société, Civilisation, 5-6, pp. 1081-1107.

Platt Tristan, Thérèse Bouysse-Cassagne et Olivia Harris

2006 Qaraqara-Charka, Mallku, Inka y rey en la provincia de Charcas (siglos $X V$-XVII). Historia de una confederación aymara, Instituto francés de estudios andinos/Plural editores/University of St. Andrews/University of London/ Inter American Foundation/Fundación Cultural del Banco Central de Bolivia, La Paz.

\section{Ponce SANGines Carlos}

1969 Tumupa y Ekako, Los Amigos del Libro, La Paz

Poole Deborah

1982 "Los sanctuarios religiosos en la economía regional andina (Cuzco) ", Allpanchis Phuturinqa, XVI (19), Cuzco, pp. 79-116.

Protzen Jean-Pierre

1994 "L'architecture Inca ", in Les Royaumes préincaïques et le monde inca, Edisud, coll. " Corpus précolombien », Paris, pp. 193-217. 


\section{Pucher De Kroll Leo}

1950 El auquenido y la cosmogonía Amerisiana, Universidad Autonoma Tomas Frías, Potosí.

Pumaccahua Rocca Esther

2005 «Estudio palinológico en los andenes del sector VIII las llamas », in Zenovio Valencia García, Helguero Gonzáles, Jorge Chávez Rodríguez, César Morante Ángeles et Abdias Calsin Ríos (éds), Investigacion histórica del complejo histórico de Choqequirao, Sector VIII, Andenes de las lamas, Proyecto Plan Copesco Nacional, Informe final, Cuzco, pp. 152-193.

RAVINES Rogger

2006 "Choquequirao : nota bene ", Boletin de Lima, XXVIII (146), pp. 25-30.

REINHARD Johan

1983 "Las montañas sagradas: un estudio etnoarqueológico de ruinas en las altas cumbres andinas ", Cuaderno de Historia, 3, Santiago, pp. 27-62.

1985 "Chavin and Tiahuanaco: a new look at two Andean ceremonial complex ", Nacional Geographic Research, 1 (3), pp. 395-422.

2002 Machu Picchu. El centro sagrado, Instituto Machu Picchu, Lima [1991].

Rembur Marie

1987 "Le tissage et les textiles », in Ancien Pérou, vie, pouvoir et mort, catalogue de l'exposition, musée de l'Homme, Paris, mai 1987-janvier 1988, Nathan, Paris, pp. 141-147.

RENARD Susana F

1994 "Vestimenta y jerarquía. Los tejidos de Angualasto del Museo Etnográfico. Una visión », Revista Andina, Año 2, diciembre, Cuzco, pp. 373-401.

RosTworowski Maria

1983 Estructuras andinas del poder, Instituto de Estudios Peruanos, Lima.

1993 "Origen religioso de los dibujos y rayas de Nazca ", Journal de la Société des Américanistes, LXXIX, pp. 189-202.

Rowe John H.

1973 "Standardization in Inca Tapestry Tunics", in The Junius B. Bird PreColumbian Textile Conference, The Textile Museum, Washington.

1978 "Technical Features of Inca Tapestry Tunics », Textile Museum Journal, 17 , pp. 5-28.

SAINTENOY Thibault

2008 "Choqek’iraw y el valle del Apurímac : hábitat y paisajes prehispánicos tardios. Una investigación en curso ", Bulletin de l'Institut français d'études andines, 37 (3), pp. 553-56.

SAMANEZ Roberto

2006 "Arquitectura de Choquequirao», Boletín de Lima, XXVIII (146), pp. 96-126.

Samanez Roberto et Julinho Zapata

1995 "El conjunto arqueológico inka de Choquequirao », Andes, 1, Cuzco, pp. 97-114. 
1999 «El centro ceremonial inka de Choquequirao », Arkinka, 46, Lima, pp. 80-94.

SARTiges Eugène

1851 "Voyage dans les républiques de l'Amérique du Sud», écrit sous le pseudonyme de E. De Lavandais, Revue des Deux Mondes, 21 (10), pp. 1019-1059.

2006 "Las ruinas de Choquequirao », Boletín de Lima, XXVII (146), pp. 35-43.

Scanu Marcelo

1987 "Sanctuarios de altura de los Andes », Revista del Museo Nacional, Lima, pp. 213-249.

SCHJELLERUP Inge

1990 "Recherches archéologiques et historiques au Chachapoya-Pérou », in IncaPerú. 3000 ans d'Histoire, musée royaux d'Art et d'Histoire, Bruxelles, pp. 236-247.

SQUiER Georges

1974 Un viaje por tierras incáicas. Crónica de una expedición arqueológica (18631865), Editorial Los Amigos del Libro, La Paz/Cochabamba [1877].

STAller John E.

2008 "Dimensions of place : the significance of centers to the development of Andean civilization. An exploration of the ushnu concept ", in John E. Staller (éd.), Pre-Columbian landscapes of creation and origin, Springer, New York, pp. 269-313.

StOBART Henry

1988 The pinkillos of Vitichi, Londres [umpublish manuscrit].

1996a "Tara and Q'iwa. Worlds of sound and meaning ", in Max Peter Baumann (éd.), Cosmología y música en los Andes, Vervuert/Iberoamericana, Berlin, pp. 67-81.

1996b "The llama's flute : musical misunderstandings in the Andes », Early Music, Londres, pp. 471-482.

TAYLOR Gérald

1974-1976 "Camay, camac et camasca dans le manuscrit quechua de Huarochiri », Journal de la Société des Américanistes, LXIII, pp. 231-244.

\section{TAYLOR Gérald (éd.)}

1980 Rites et traditions de Huarochirí : manuscrit quechua du début du XVIf siècle, Éditions l'Harmattan, coll. « Ethnolinguistique Amérindienne », Paris.

\section{TOMOEDA Hiroyasu}

1993 "Los ritos contemporáneos de camélidos y la ceremonia de la citua ", in Luis Millones et Yoshio Onuki (éds), El Mundo ceremonial andino, National Museum of Ethnology, coll. « Ethnological Studies » 37, Osaka, pp. 289-306.

1996 "The concept of vital energy among Andean pastoralists », in Ellen Roy et Fukui Katsuyoshi (éds), Redefining nature, ecology, culture and domestication, Berg, Oxford/Washington DC, pp. 186-212. 
1998 «El mundo del color y del movimiento : de los takis precolombinos a los danzantes de tijeras ", in Walter Wust et Germán Coronado (éds), Historia, religión y ritual de los pueblos ayacuchanos, National Museum of Ethnology, coll. « Ethnological Report » 9, Osaka, pp. 129-141.

TschopIK Harry Jr.

1946 "The Aymara ", in Julian Steward (éd.), Handbook of South American Indians, vol. 2. The Andean civilizations, Smithsonian Institution, Bureau of American Ethnology, bulletin 143, Washington DC, pp. 501-574.

UCEDA Santiago, Elias Mujica et Ricardo Morales (éds)

2006 Investigaciones en la Huaca de la Luna 2000, Facultad de Ciencias Sociales, Universidad de Trujillo, Trujillo.

URBANO Enrique

1993 "La figura y la palabra. Introducción al estudio del espacio simbólico andino ", in Enrique Urbano (éd.), Mito y simbolismo en los Andes. La figura y la palabra, Centro Bartolomé de las Casas, Cuzco, pp. 7-50.

URTON Gary

2003 La vida social de los numeros. Una ontologia de los numeros y la filosofia de la aritmética quechuas, avec la collaboration de Nina Llanos, Centro Bartolomé de las Casas, Cuzco [1997].

2006 En el cruce de rumbos de la tierra y el cielo, Centro Bartolomé de las Casas, Cuzco [1981].

Valencia García Zenovio

2004 "Pinturas rupestres de Negruyoc », Primero simposio nacional de Arte Rupestre, Cuzco, communication orale [manuscrit].

Valencia García Zenovio, Jorge Chávez Rodríguez, César Morante Ángeles et Abdias CaLsin Ríos

2005 Investigación histórica del complejo arqueológico de Choquequira, Sector VIII, Andenes de las llamas, Informe Final 2005, Ministerio de Comercio Exterior y Turismo Mincetur, Proyecto Plan Copesco Nacional, Cuzco.

VAN DE Guchte Maarten J.

1990 Carving the world: Inca monumental sculpture and landscape, $\mathrm{PhD}$ dissertation, University of Illinois at Urbana Campaign, Campaign Ill.

WALTER Doris

2003 La domestication de la nature dans les Andes péruviennes. L'alpiniste, le paysan et le parc national du Huascarán, L'Harmattan, Paris.

WEST Terry

1979 Sufriendo nos vamos: from a subsistance to a market economy in an Aymará community of Bolivia, PhD dissertation, University of Michigan, New School for Social Research, Ann Arbor, Michigan.

WIENER Charles

1983 Perú y Bolivia : relato de viaje, Instituto francés de etudios andinos, coll. "Travaux de l'Institut français d'études andines » 56/Universidad Mayor San Marcos, Lima [1880]. 
WiLliams Véronica

2008 "Espacios conquistados y símbolos materiales del imperio inca en el Norte de Argentina », in Paola Gonzáles Carvajal et Tamara Bray (éds), Lenguajes. Visualesdelosincas, British Archeological Report, $\mathrm{n}^{\circ}$ 1848, Oxford, pp. 47-70.

Wright Véronique

2007 Étude de la polychromie des reliefs sur terre crue du Huaca de la Luma, Trujillo, Pérou, thèse de doctorat de l'université Paris 1, Paris.

ZAPATA Julinho

2005 "Paysages, Ancêtres et Eau Sacrée à Choquequirao », in Choqequirao. Le mystère des lamas du Soleil et le culte aux Apus, Fond Contre-valeur PérouFrance, Lima, pp. 93-124.

2006 "Choquequirao, sanctuario inca ", in Raices vivas del Perí, Servicios Editoriales del Perú, Lima, pp. 96-121.

\section{Zeguarra Choque Erlinda et Eduardo Puma Laime}

2000 Danza de los wauqos en la fiesta de la Virgen de Guadalupe, Universiteit "Gent », Belgique, Universidad Autónoma Tomas Frías, Potosí.

ZORN Elayne

1987 "Un análisis de los tejidos en los atados rituales de los pastores », Revista Andina, 2, diciembre, Cuzco, pp. 489-526.

\section{ZUIDEMA TOM}

1974-1976 « La imagen del sol y la huaca de Susurpuquio en el sistema astronómico de los incas en el Cuzco », Journal de la Société des Américanistes, LXIII, pp. 199-230.

1978 "Lieux sacrés et irrigation: tradition historique, mythe et rituels au Cuzco ", Annales. Économie, Société et Civilisation, 5-6, septembredécembre, pp. 1037-1056.

1989 "El Ushnu », in Reyes y guerreros. Ensayos de cultura andina, Grandes Estudios Andinos, Fomciencias, Lima, pp. $402-454$ [1980].

1992 "The organization of sacrifice in the city of Cuzco ", Joumal of Latin American Lore, 18, Berkeley, pp. 63-78.

1995 El sistema de Ceque del Cuzco, Pontificia Universidad Católica del Perú, Fondo Editorial, Lima.

2005 "Tiwanaku Iconography and the Calendar ", in Margaret Young-Sánchez (éd.), Tiwanaku : papers from the 2005 Mayer center symposium at the Denver Art Museum, University of Oklahoma Press, pp. 83-99.

2008a "The astronomical significance of ritual movements in the calendar of Cuzco ", in John E. Staller (éd.), Pre-Columbian landscapes of creation and origin, Springer, New York, pp. 249-267.

2008b "El sistema de ceques como computadora », in Paola González Carvajal et Tamara L. Bray (éds), Lenguajes. Visuales de los incas, British Archeological Report, $\mathrm{n}^{\circ}$ 1848, Oxford, pp. 103-109.

Zuidema Tom et Gary UrToN

1976 "La constelación de la Llama en los Andes peruanos », Allpanchis Phuturinqa, IX (9), Cuzco, pp. 59-150. 